University of Louisville

ThinkIR: The University of Louisville's Institutional Repository

\title{
A forward dynamics simulation study of increasing load on the anterior cruciate ligament of the knee, for young women performing recreational drop jump activities.
}

Julia Kar

University of Louisville

Follow this and additional works at: https://ir.library.louisville.edu/etd

\section{Recommended Citation}

Kar, Julia, "A forward dynamics simulation study of increasing load on the anterior cruciate ligament of the knee, for young women performing recreational drop jump activities." (2011). Electronic Theses and Dissertations. Paper 724.

https://doi.org/10.18297/etd/724

This Doctoral Dissertation is brought to you for free and open access by ThinkIR: The University of Louisville's Institutional Repository. It has been accepted for inclusion in Electronic Theses and Dissertations by an authorized administrator of ThinkIR: The University of Louisville's Institutional Repository. This title appears here courtesy of the author, who has retained all other copyrights. For more information, please contact thinkir@louisville.edu. 


\title{
A FORWARD DYNAMICS SIMULATION STUDY OF INCREASING LOAD ON THE ANTERIOR CRUCIATE LIGAMENT OF THE KNEE, FOR YOUNG WOMEN PERFORMING RECREATIONAL DROP JUMP ACTIVITIES
}

\author{
By \\ Julia Kar \\ B.E. University of Auckland, 1996 \\ M.S. University of Louisville, 2008

\begin{abstract}
A Dissertation
Submitted to the Faculty of the

Speed School of Engineering, University of Louisville

for the Degree of
\end{abstract} \\ Doctor of Philosophy \\ Department of Mechanical Engineering \\ University of Louisville \\ Louisville, Kentucky
}

May 2011 
Copyright $^{(0} 2011$ by Julia Kar

All Rights Reserved 


\title{
A FORWARD DYNAMICS SIMULATION STUDY OF INCREASING LOAD ON THE ANTERIOR CRUCIATE LIGAMENT OF THE KNEE, FOR YOUNG WOMEN PERFORMING RECREATIONAL DROP JUMP ACTIVITIES
}

By

\author{
Julia Kar \\ B.E., University of Auckland, 1995 \\ M.S., University of Louisville, 2008
}

A Dissertation Approved on

April 122011

by the following Dissertation Committee:

Dissertation Director (Peter M. Quesada) 


\section{DEDICATION}

This dissertation is dedicated to my family. 


\section{ACKNOWLEDGEMENT}

I acknowledge the help of my family, mentors, and advisors. 


\title{
ABSTRACT \\ A FORWARD DYNAMICS SIMULATION STUDY OF INCREASING LOAD ON THE ANTERIOR CRUCIATE LIGAMENT OF THE KNEE, FOR YOUNG WOMEN PERFORMING RECREATIONAL DROP JUMP ACTIVITIES
}

\author{
Julia Kar
}

April 12, 2011

Anterior Cruciate Ligament (ACL) injuries are among the most common injuries incurred by both recreational and professional athletes. ACL injuries often occur during popular contact sports like basketball, football, volleyball and baseball, and non-contact activities like aerobics, jogging and running. Non-contact actions like jumping, sprinting and sidecutting that involve sudden or rapid changes in motion may lead to ACL injuries. At the instance of an injury, the knee joint muscles and ligaments typically undergo extremely high loads. The ACL, which is an integral part of the knee joint undergo high strain rates and rapid energy absorption, and consequently get injured. As has been shown by others, ACL injury is related to a number of dynamic variables of the knee joint.

An important observation made in recent years is that recreational (also professional) female athletes have higher incidences of noncontact ACL injuries than males ${ }^{33,35}$. The primary focus of this study was to determine effects of several dynamic variables, associated with both knee and ACL, during normal recreational drop-jump activities performed by young female athletes. Subjects recruited were eleven young adult female recreational athletes who felt comfortable participating in the drop-jump activities, from 
heights of 30,40 and $50 \mathrm{~cm}$. Using a simulation environment to recreate the trials, changes in ACL load and strain were observed along with several dynamic variables related to ACL load and strain, among which the three most important were, 1. knee flexion, 2. knee valgus (abduction) which may be accompanied by increased internal rotation, and, 3. flexor to extensor muscle recruitment ratios, i.e., the co-contraction of flexor and extensor muscles.

Observations from the above simulations formed the basis of the final step involving forward dynamic simulation, where the knee joint was subject to higher valgus by decreasing the distance between the knees (medial translation). Significant changes to ACL load and strain were seen in the added medial translation simulations compared to the simulations from the original jumps. Mean fiber strain for the additional valgus simulation increased from $8.82 \pm 0.08 \%$ to $11.82 \pm 0.04 \%$ for the right ACL and from $8.18 \pm 0.08 \%$ to $11.34 \pm 0.06 \%$ for the left. Mean ACL tensile force increased from $1058.19 \pm 2.04 \mathrm{~N}$ to $1102.19 \pm 1.86 \mathrm{~N}$ for the right $\mathrm{ACL}$ and from $1056.77 \pm 12.36 \mathrm{~N}$ to $1099.99 \pm 2.02 \mathrm{~N}$ for the left. Average peak (from eleven subjects) ACL tensile force increased from $1165.36 \pm 123.83 \mathrm{~N}$ to $1197.07 \pm 129.11 \mathrm{~N}$ for the right ACL and from $1160.64 \pm 121.32 \mathrm{~N}$ to $1193.11 \pm 130.16 \mathrm{~N}$ for the left. 


\section{TABLE OF CONTENTS}

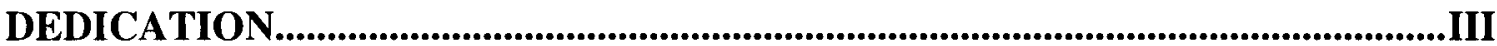

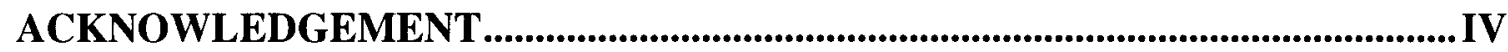

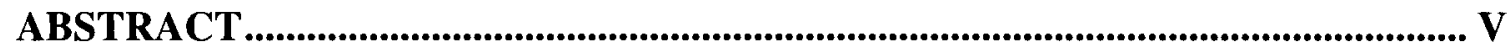

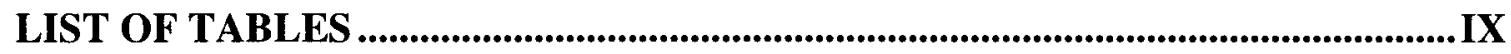

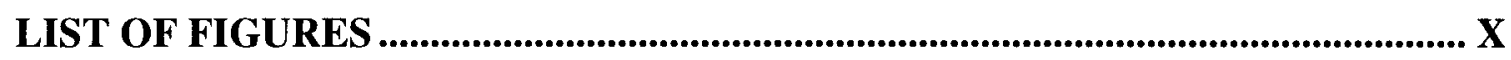

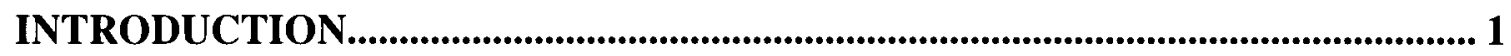

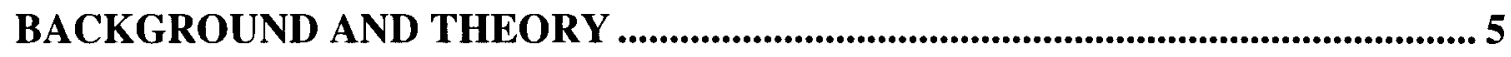

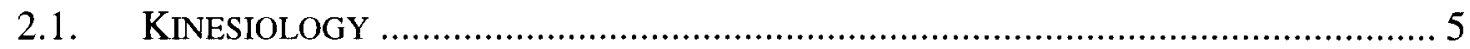

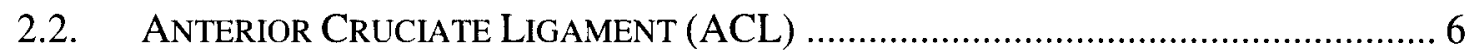

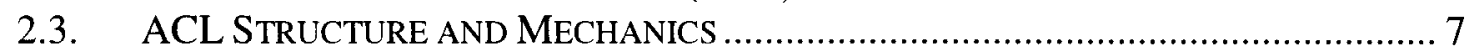

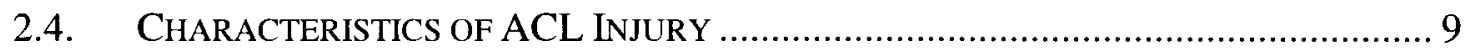

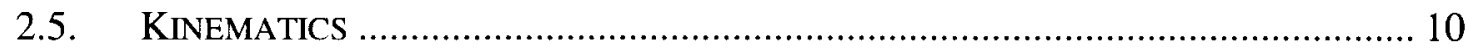

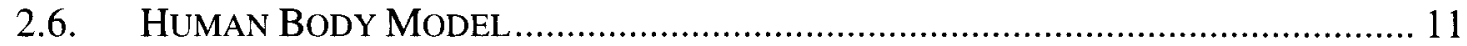

2.7. JOINT AND SEGMENT KINEMATICS .............................................................. 13

2.8. COLLECTION OF THREE DimENSIONAL Kinematic DATA .................................. 15

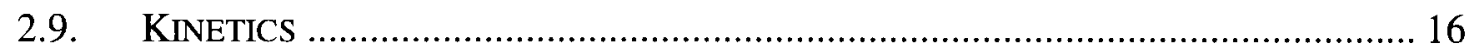

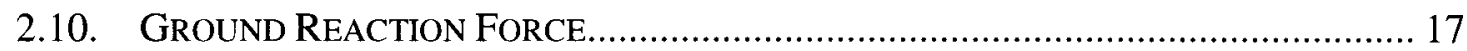

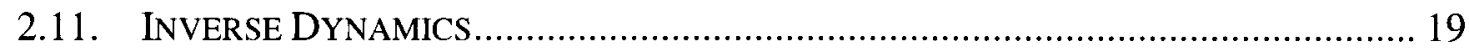

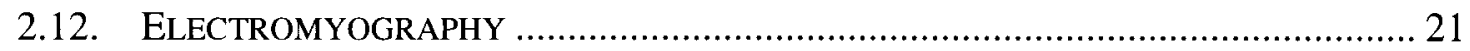

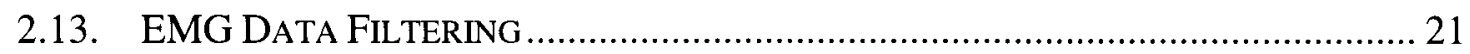

2.14. JOINT AND SEGMENT POSITION FROM INVERSE KINEMATICS ............................ 23

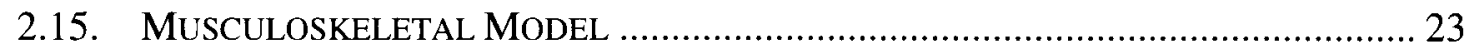

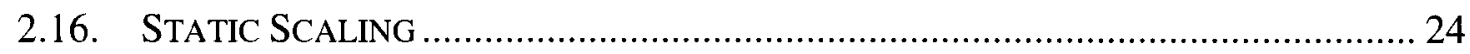

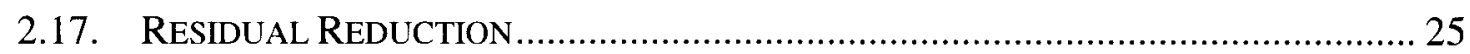

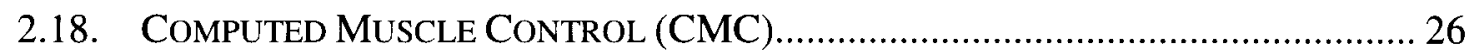

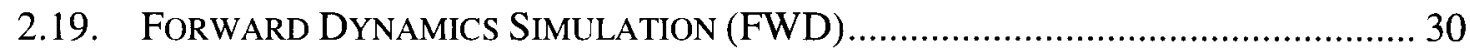

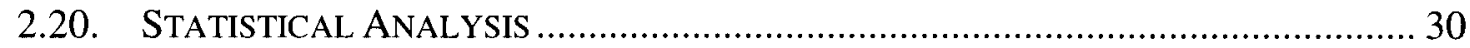

EXPERIMENTAL METHOD AND ANALYSIS.................................................. 32

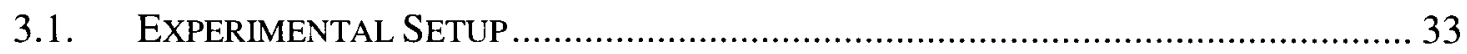

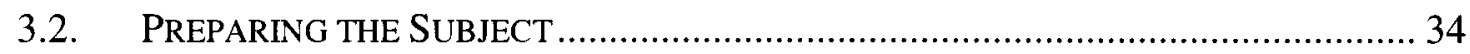

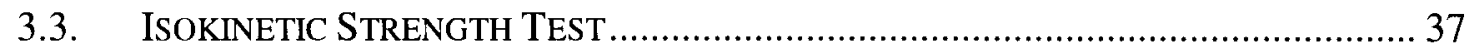

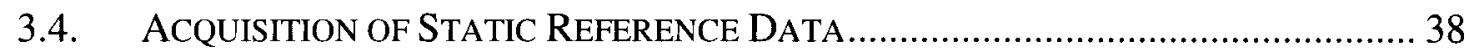

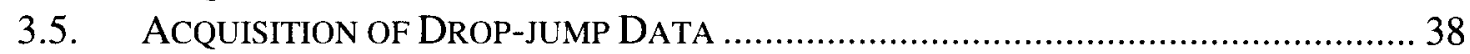

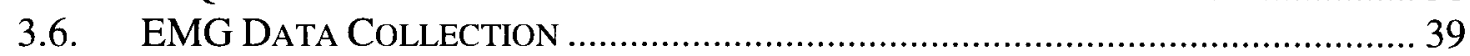


3.7. A BRIEF DESCRIPTION OF ELECTRODE FUNCTION ........................................ 41

3.8. POST-PROCESSING OF ACQUIRED DATA ............................................... 42

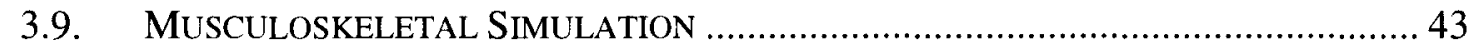

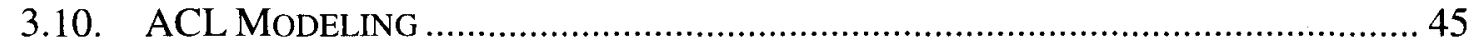

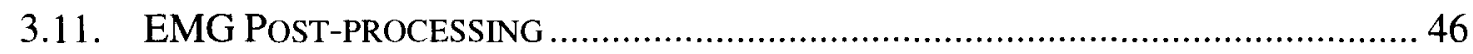

3.12. SIMULATING INCREASED KNEE VALGUS DURING LANDING........................... 48

3.13. MEASUREMENT OF ACL STRAIN ........................................................... 49

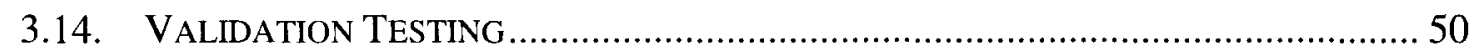

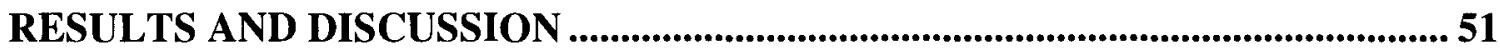

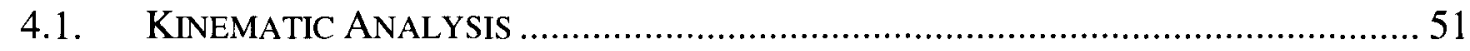

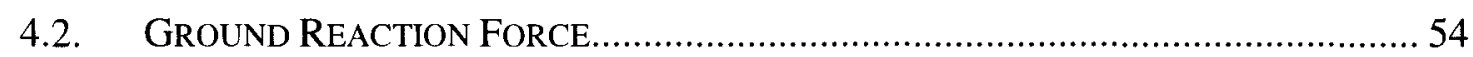

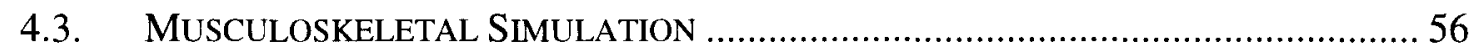

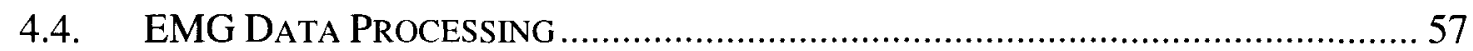

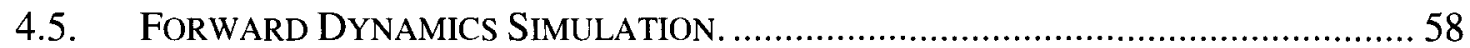

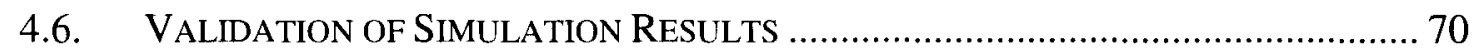

4.7. RESULTS FROM SIMULATIONS WITH INCREASED KNEE VALGUS ......................... 75

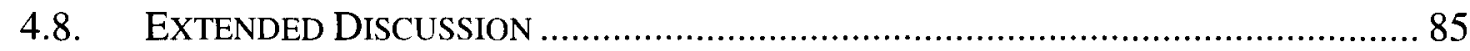

CONCLUSION

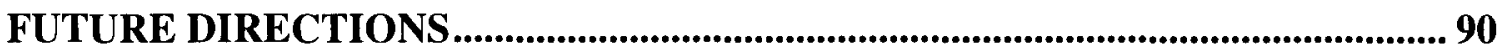

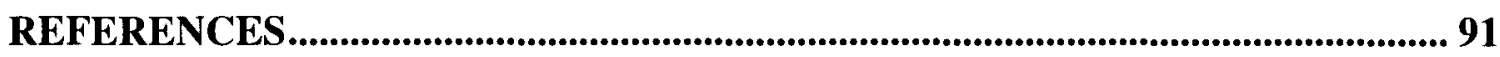

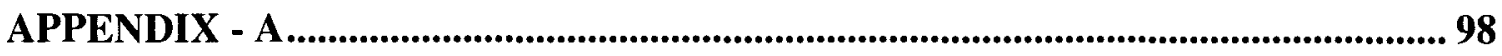

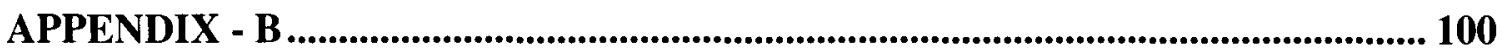

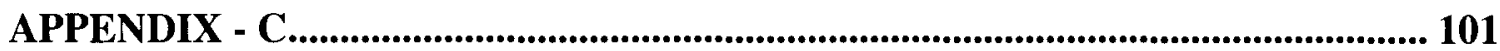

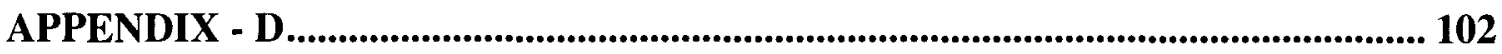

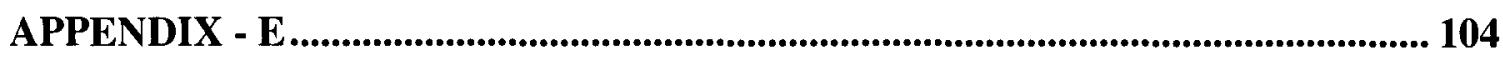

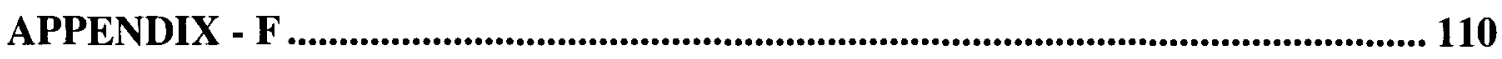

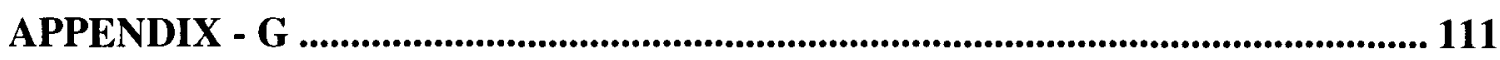

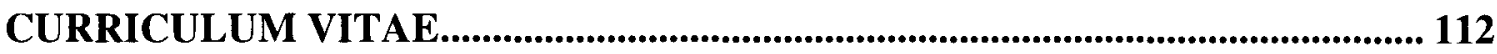




\section{LIST OF TABLES}

TABLE 1: KINEMATIC AND KINETIC FACTORS OF KNEE BEHAVIOR CLOSELY ASSOCIATED WITH POSSIBLE INCREASE IN ACL LOADING.. ............................................. 42

Table 2. Peak vertical ground ReACtion forces AVERAGed among ALl EleVen

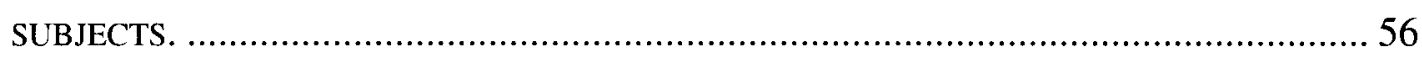

TABle 3: PEAK ground REACTION ABduction MOMENTS AVERAGED AMONG ALL ELEVEN

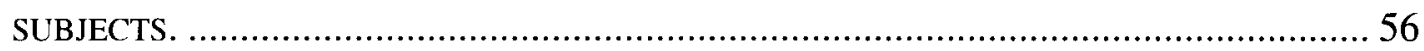

TABLE 4. AVERAGE BODY SEGMENT MASSES FROM 11 SUBJECTS. .................................. 57

TABLE 5. COEFFICIENTS OF CORRELATION BETWEEN EXPERIMENTAL AND SIMULATED HQ

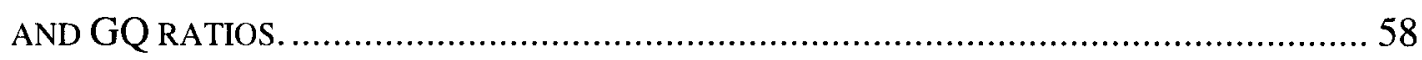

TABle 6. AVERAge PEAK ACL FIBER STRAIN FOR 30, 40 AND 50 CM JUMP HEIGHTS

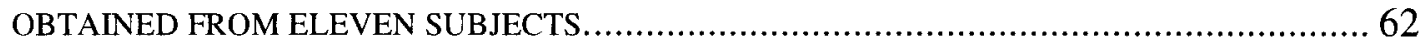

TABle 7. AVERAGE PEAK FIBER FORCE FOR 30, 40 AND 50 CM JUMP HEIGHTS OBTAINED

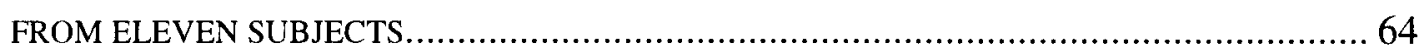

TABle 8. Correlation COEFFICIENT Between AVERAgE FiBER FORCE AND

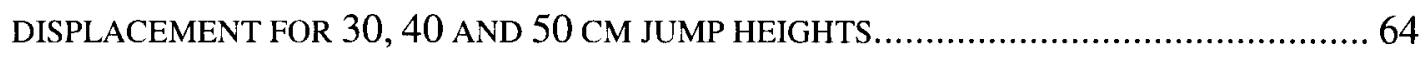

TABLE 9. AVERAGE PEAK KNEE MOMENTS FOR 30, 40 AND 50 CM JUMP HEIGHTS OBTAINED

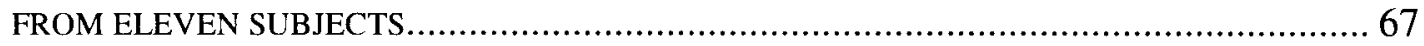

TABLE 10. CORRELATION COEFFICIENTS BETWEEN KNEE MOMENTS AND KNEE ANGLES FOR

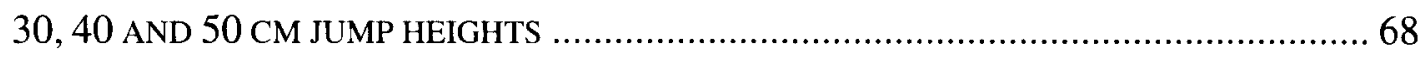

TABLE 11. PEARSON CORRELATION COEFFICIENT AND COEFFICIENT OF DETERMINATION FOR MODEL VALIDATION BY COMPARING FLEXION AND VALGUS JOINT ANGLES FROM INVERSE KINEMATICS AND FORWARD DYNAMICS. [A]: RESUlTS FROM SUBJECT A (ANONYMOUS) AND [B]: RESUlTS FROM SUBJECT B (ANONYMOUS) EXECUTING A

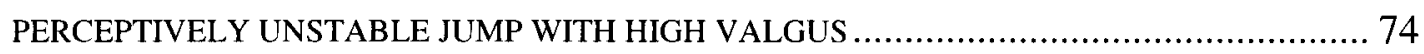




\section{LIST OF FIGURES}

Figure 1. SAgitTAl MRI image of THE KNEE JOINT SHOWING THE ANTERIOR CRUCIATE LigAMENT AND POSTERIOR CRUCIATE LigAMENT. 3

FigURE 2. LOCATION OF MAIN LIGAMENTS AND BONES OF THE KNEE. TOP SURFACE OF TIBIA SHOWING WHERE BOTH ANTERIOR CRUCIATE LIGAMENT (ACL) AND POSTERIOR CRUCIATE LIGAMENT (PCL) ATTACH. ....................................................... 6

FiguRE 3. [A]: FRONT OF KNEE WITH THE QUADRICEPS MUSCLES: [B]. BACK OF THE KNEE WITH THE GASTROCNEMIUS MUSCLES AND THE HAMSTRING MUSCLE. ...................... 7

FigurE 4. [A]: LIGAMENT HIERARCHICAL STRUCTURE WITH FIBRILS AND COLLAGEN SHOWING THE CRIMP IN FASCICLES OR FIBRIL BUNDLES. [B]: LOAD DEFORMATION CURVE SHOWING NONLINEAR BEHAVIOR FROM AN IN-VITRO RABBIT LIGAMENT.......... 8

FIGURE 5. [A]: ORIENTATION OF THE GLOBAL REFERENCE COORDINATES SYSTEM (GCS). WITH MAIN DIRECTIONS OF TRANSLATION AND MAIN PLANES OF ROTATION. [B]: THE MOMENTS AT THE THREE MAIN JOINTS OF THE LOWER BODY. 11

Figure 6. THREE MAIN SEGMENTS OF THE LOWER EXTREMITIES. FORCES AND MOMENTS ARE NAMED ACCORDING TO THE JOINT AND SEGMENT NAMES WHERE THEY ACT ....... 12

FigURE 7. TRANSFORMATION FROM THE [X Y Z] SYSTEM TO THE [X $\square \mathrm{Y} \square \mathrm{Z} \square]$ SYSTEM USING THE CARDAN SYSTEM OF ROTATION.

Figure 8. TyPiCAL FORCE PLATE SHOWING THE FORCE PLATE'S LOCAL COORDINATE SYSTEM AND THE MOTION ANALYSIS GLOBAL COORDINATE SYSTEM.. 18

FIGURE 9. LOW PASS BUTTERWORTH FILTER SHOWING GAIN $\mathrm{H}_{\mathrm{C}}$ AT DIFFERENT POLES OF $\mathrm{N}=2,4$, AND 8 .. 22

FIGURE 10. MUSCULOSKELETAL MODEL SHOWING MAIN BODY SEGMENTS (IN BOLD) AND MUSCLES THAT CONTRIBUTE TOWARDS KNEE MOTION. 25

Figure 11. SCHEMATiC OF Close loOPEd COMPuTEd MusCle CONTROL (CMC) PRoCESS

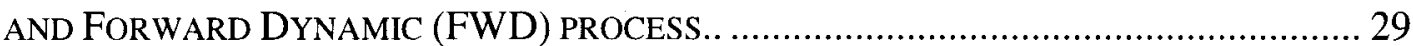

FIGURE 12. FLOW CHART OF SEQUENCE OF TASKS FOR TO THE PROJECT. 33 
FIGURE 13. [A]. MARKER PLACEMENTS ON THE WHOLE BODY FOR STATIC TRIALS........... 35

FIGURE 13. [B]. MARKER PLACEMENT ON THE WHOLE BODY FOR DYNAMIC TRIALS.. ....... 36

FigURE 14. PlaCEMENT OF EMG SENSORS ON MUSCLES OF THE LOWER EXTREMITY ....... 37

FIGURE 15. EMG ARRANGEMENT WITH THE ELECTRODES, AMPLIFIERS, HOOK-UP CABLES AND HAND (HIP) HELD MONITOR WITH WIRELESS CONNECTION TO THE HOST. 40

Figure 16. DELSYS DiFFERENTIAL ELECTRODE PLACED ON MUSCLE TISSUE (PINK). EMG SIGNAL IS THE RESULT OF POTENTIAL DIFFERENCE BETWEEN THE ELECTRODES 41

FIGURE 17. FLOW CHART SHOWING SEQUENCE OF TASKS FOR DYNAMIC SIMULATION OF THE MUSCULOSKELETAL MODEL 43

FIGURE 18. MusCUlOSKELETAL MODEL SHOWING THE 22 ROTATIONAL DEGREES OF FREEDOM AND 3 TRANSLATIONAL DEGREES OF FREEDOM. 43

Figure 19. ForCE Plate CONTACT DYNAMICS OF A JUMP TRAJECTORY (30 CM JUMP HEIGHT) USING OPENSIM 2.20 GRAPHICAL INTERFACE SHOWING GROUND REACTION FORCE (GREEN) AND VARIOUS STANCES IN CHRONOLOGICAL SEQUENCE, [A]: TOUCH DOWN, [B]: MAXIMUM CROUCH, [C]: RECOVERING, [D]: RECOVERED 45

FIGURE 20. [A]: FRONTAL VIEW OF THE ACL WITH INSERTION POINTS INTO THE INTERCONDYLOIDIC EMINENCE (FEMUR) AND THE FRONT MENISCULES (TIBIA). [B]: RIGHT SAGITTAL VIEW OF THE ACL. 46

Figure 21. Front PANEL OF NATIONAL INSTRUMENTS LABVIEW ${ }^{\mathrm{TM}}$ VI FOR FILTERING DATA FROM EXPERIMENTS. THE PLOT SHOWS PRE AND POST-FILTERED EMG FOR A SINGLE TRIAL. 48

Figure 22. [A]: ORIGINAL 50 CM STANCE AND [B]: VALgUS INCREASE 50 CM STANCE By DECREASING THE HORIZONTAL DISTANCE BETWEEN KNEES. 49

FIGURE 23. GENERAL POSITIONS OF FEMUR AND TIBIA SHOWING THE DIRECTION IN WHICH KNEE IS BENT TO DETERMINE CHANGES IN ACL LENGTH UNDER TOMOGRAPHY . ....... 50

FIGURE 24. RIGHT AND LEFT KNEE FLEXION ANGLES AGAINST PERCENTAGE TIME OBTAINED FOR ONE SUBJECT FOR JUMP HEIGHTS OF 30 CM, 40 CM AND 50 CM....................... 53

FIGURE 25. RIGHT AND LEFT KNEE VALGUS ANGLES AGAINST PERCENTAGE TIME OBTAINED FOR ONE SUBJECT FOR JUMP HEIGHTS OF 30 CM, 40 CM AND 50 CM......................... 54

FigURE 26. RIGHT AND LEFT KNEE VERTICAL GRF FORCES AGAINST PERCENTAGE TIME FOR ONE SUBJECT FOR JUMP HEIGHTS OF $30 \mathrm{CM}, 40 \mathrm{CM}$ AND $50 \mathrm{CM}$ 55 
FigURE 27. AVERAGE RIGHT AND LEFT ACL FIBER LENGTHS AGAINST PERCENTAGE TIME OBTAINED FROM ELEVEN SUBJECTS PARTICIPATING IN THE DROP-JUMP TRIALS ......... 59

Figure 28. AVERAGE RIGHT AND LEFT ACL STRAINS AGAINST PERCENTAGE TIME OBTAINED FROM ELEVEN SUBJECTS PARTICIPATING IN THE DROP-JUMP TRIALS ......... 60

Figure 29. AVERAGE RIGHT AND LEFT ACL STRAIN RATES AGAINST PERCENTAGE TIME OBTAINED FROM ELEVEN SUBJECTS PARTICIPATING IN THE DROP-JUMP TRIALS 61

FigurE 30. AVERAGE RIGHT AND LEFT ACL FORCES AGAINST PERCENTAGE TIME OBTAINED FROM ELEVEN SUBJECTS PARTICIPATING IN THE DROP-JUMP TRIALS 63

FigurE 31. AVERAGE RIGHT AND LEFT KNEE FLEXION MOMENTS AGAINST PERCENTAGE TIME OBTAINED FROM ELEVEN SUBJECTS PARTICIPATING IN THE DROP-JUMP TRIALS..65

FIGURE 32. AVERAGE RIGHT AND LEFT KNEE VALGUS MOMENTS AGAINST PERCENTAGE TIME OBTAINED FROM ELEVEN SUBJECTS PARTICIPATING IN THE DROP-JUMP TRIALS..66

Figure 33. AVERAGE RIGHT AND LEFT KNEE FLEXION POWER AGAINST PERCENTAGE TIME OBTAINED FROM ELEVEN SUBJECTS PARTICIPATING IN THE DROP-JUMP TRIALS 69

Figure 34. AVERAGE RIGHT AND LEFT KNEE VALGUS POWER AGAINST PERCENTAGE TIME OBTAINED FROM ELEVEN SUBJECTS PARTICIPATING IN THE DROP-JUMP TRIALS ......... 70

FIGURE 35. RIGHT AND LEFT KNEE FLEXION VS TIME FOR SUBJECT A CALCULATED WITH IK AND FORWARD DYNAMICS ANALYSIS. 71

FIGURE 36. RIGHT AND LEFT KNEE FLEXION VS TIME FOR SUBJECT B CALCULATED WITH IK AND FORWARD DYNAMICS ANALYSIS. 72

FigURE 37. Right AND LEFT KNEE VALGUS VS TIME FOR SUBJECTS A AND B CALCULATED WITH IK AND FORWARD DYNAMICS ANALYSIS. 73

FIGURE 38. AVERAGE RIGHT AND LEFT KNEE FLEXION ANGLES AGAINST PERCENTAGE TIME OBTAINED FROM SIMULATIONS OF THE ORIGINAL 50 CM JUMP AND 50 CM JUMP WITH ADDED MEDIAL TRANSLATION (VALGUS) 76

FIGURE 39. AVERAGE RIGHT AND LEFT KNEE VALGUS ANGLES AGAINST PERCENTAGE TIME OBTAINED FROM SIMULATIONS OF THE ORIGINAL $50 \mathrm{CM}$ JUMP AND 50 CM JUMP WITH ADDED MEDIAL TRANSLATION (VALGUS) 76

Figure 40. AVERAGE SIMULATEd HQ AND GQ RATIO From SIMUlations OF THE ORIGINAL 50 CM JUMP AND 50 CM JUMP WITH ADDED MEDIAL TRANSLATION. 78 
Figure 41. AVERAGE RIGHT AND LEFT ACL FIBER LENGTHS FROM SIMULATIONS OF THE ORIGINAL 50 CM JUMP AND 50 CM JUMP WITH ADDED MEDIAL TRANSLATION........... 79

FIGURE 42. AVERAGE RIGHT AND LEFT ACL FIBER STRAINS FROM SIMULATIONS OF THE ORIGINAL 50 CM JUMP AND 50 CM JUMP WITH ADDED MEDIAL TRANSLATION. 80

FIGURE 43. AVERAGE RIGHT AND LEFT ACL FIBER STRAIN RATES FROM SIMULATIONS OF THE ORIGINAL 50 CM JUMP AND 50 CM JUMP WITH ADDED MEDIAL TRANSLATION..... 80 Figure 44. AVERAGE RIGHT AND LEFT ACL FIBER FORCES FROM SIMULATIONS OF THE ORIGINAL 50 CM JUMP AND 50 CM JUMP WITH ADDED MEDIAL TRANSLATION. 81

FIGURE 45. AVERAGE RIGHT AND LEFT FLEXION KNEE MOMENTS FROM SIMULATIONS OF THE ORIGINAL 50 CM JUMP AND 50 CM JUMP WITH ADDED MEDIAL TRANSLATION..... 83 FIGURE 46. AVERAGE RIGHT AND LEFT KNEE VALGUS MOMENTS FROM SIMULATIONS OF THE ORIGINAL 50 CM JUMP AND 50 CM JUMP WITH ADDED MEDIAL TRANSLATION............83 FIGURE 47. AVERAGED RIGHT AND LEFT KNEE FLEXION POWER FROM SIMULATIONS OF THE ORIGINAL 50 CM JUMP AND 50 CM JUMP WITH ADDED MEDIAL TRANSLATION 84

FIGURE 48. AVERAGED RIGHT AND LEFT KNEE VALGUS POWER FROM SIMULATIONS OF THE ORIGINAL $50 \mathrm{CM}$ JUMP AND $50 \mathrm{CM}$ JUMP WITH ADDED MEDIAL TRANSLATION 85

FIGURE 49. FORCE-LENGTH-VELOCITY CURVE OF AN IDEALIZED MUSCLE SHOWING ONLY THE CONCENTRIC PHASE OF CONTRACTION 100

Figure 50. Hill-HuXley MODEL OF MUSCLO-TENDON CONTRACTION DYNAMICS, AS MODIFIED AND USED IN OPENSIM $2.20^{64}$. 101

FIGURE 51. AVERAGE RIGHT AND LEFT GRF FORCES VS TIME IN THE X DIRECTION. ........ 104 FIGURE 52. AVERAGE RIGHT AND LEFT GRF FORCES VS TIME IN THE Y DIRECTION. ....... 105 FIGURE 53. AVERAGE RIGHT AND LEFT GRF FORCES VS TIME IN THE Z DIRECTION......... 106 FigURE 54. AVERAGE RIGHT AND LEFT GRF MOMENTS VS TIME IN THE X DIRECTION. ... 107 FIGURE 55. AVERAGE RIGHT AND LEFT GRF MOMENTS VS TIME IN THE Y DIRECTION. ... 108 FIGURE 56. AVERAGE RIGHT AND LEFT GRF MOMENTS VS TIME IN THE Z DIRECTION...... 109 FIGURE 57. PEAK PROPULSION GRF RELATIVE TO BODY WEIGHT . 110 FIGURE 58. LENGTH-TENSION RELATION OF A (RABBIT) LIGAMENT WHEN STRETCHED AT DIFFERENT RATES. 111 


\section{CHAPTER I}

\section{INTRODUCTION}

Today there is an increasing demand to understand human movement in daily activities like work, sports and recreation. Human movement science studies the bio-mechanical aspects of motion and often involves a combined study of muscle physiology and neurological synapses that are essential to human motion. Studying how the human body reacts (kinematics) to its surrounding environment and external stimuli also contribute to the science of human movement.

Along with everyday motion, aspects of injuries, disabilities and diseases are essential parts of human movement science. Finding effective methods for prevention of injury and rehabilitation has now become important also. Research in this area is known as injury biomechanics. It generally focuses on studying excessive deformation or strain incurred by a particular segment of the human anatomy, for example, rupturing the knee ACL during intense lower extremity activities.

More than 200,000 new ACL injuries occur in the United States each year, either from direct or indirect loading of the knee ${ }^{35,39,42,45}$, of which estimated $38,000{ }^{33}$ happen to

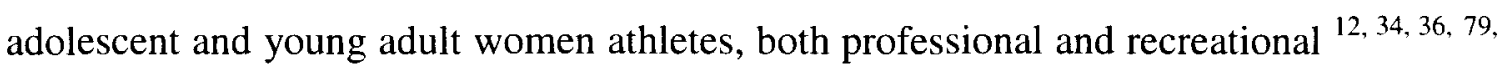
${ }^{80}$. Around $70 \%$ to $80 \%$ of ACL injuries happen due to indirect loads and are classified as 
non-contact ${ }^{35,48}$. It is conceived that one common form of non-contact ACL injury happens when athletes land in a knock-knee position during drop-jumps. Other than the knock-knee non-contact condition, ACL injury may also happen when athletes stop suddenly or perform twisting or cutting actions ${ }^{30,33,34}$. Often the athlete will hear or feel a "pop" at the time of injury and sometimes they may experience brief hyperextension of the knee joint. Victims are generally unable to continue activity right after injury and the injured knee swells considerably.

Physical examination for ACL injury includes the Lachman Physical Examination and Anterior Drawer tests. Radiographic images and MRIs are also used to confirm ACL injury diagnoses. Figure 1 (over the page) shows the MRI image of an intact ACL and also the Posterior Cruciate Ligament (PCL) that interacts with the ACL in balancing the knee. ACL injuries range from a partial tear to a complete tear to bone avulsion. Concurrent meniscal injuries are also common.

Partial and complete ACL tears are repaired with sutures or reconstructed using a graft. In these situations, bone avulsions are likely to happen with tearing away of bony fragments. Both surgical and non-surgical treatments are available to patients. Nonsurgical treatment is generally an option for patients with little or no athletic participation and for patients unable to participate in post-surgery rehabilitation. However, if nonoperative treatment fails, the only option left is that of operative treatment. Estimated cost of surgery per injury is approximately $\$ 17,000^{33}$. 


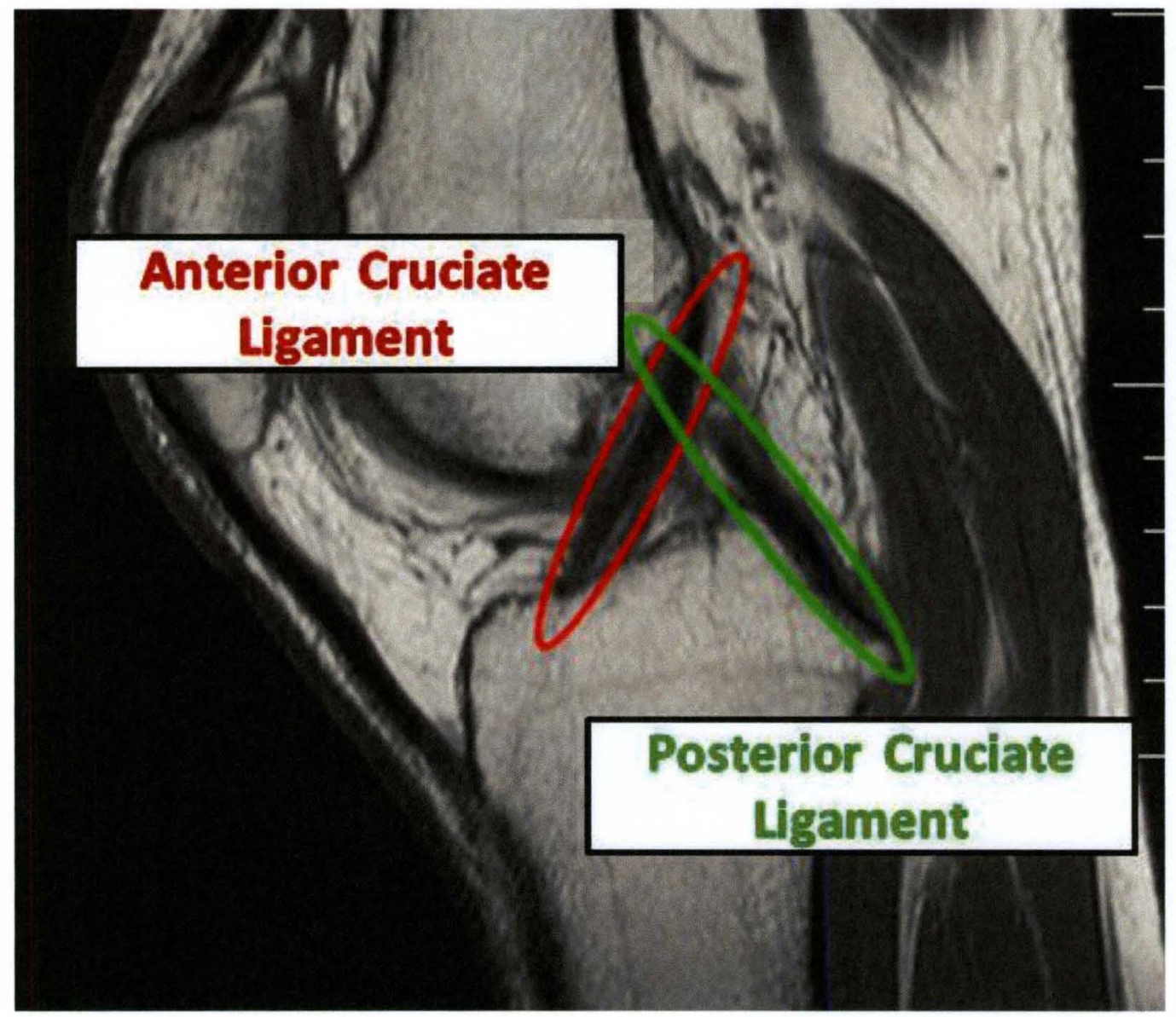

Figure 1. Sagittal MRI image of the knee joint showing the Anterior Cruciate Ligament and Posterior Cruciate Ligament.

Courtesy: Free media at www.wikipedia.org.

While there are well developed diagnostic and rehabilitation techniques for ACL injury, prevention of ACL injury is still in its primitive stage. In order to understand ACL injury, a close investigation of the dynamic variables ${ }^{62,63,64,70,73}$ that contribute toward or cause elevated loading of the ACL, was required. While everyday activities are studied in a human movement laboratory, one cannot expect to reproduce dangerous injuries within the laboratory. Therefore one must rely on the athlete's normal performance and leave the case of excessive loading to simulation studies. Accordingly for this study, athletes 
performed drop-jumps under circumstances of reasonably safe conditions. As subjects performed the drop-jumps from different heights, the dynamic variables related to ACL and surrounding muscles were monitored. These variables were then used to reproduce the drop-jumps within a simulation environment. The ultimate goal was to determine the loading effect on ACL and surrounding muscles, by changing the related variables, inside the safe simulation environment. The simulation was accomplished using a forward dynamics tool from OpenSim $2.20{ }^{64}$ which has the capability to generate results through a highly optimized sparse solver. 


\section{CHAPTER II}

\section{BACKGROUND AND THEORY}

Before the actual test setup and procedure are discussed, certain terms, concepts and current technologies utilized here will be introduced and discussed. The first part of this chapter explains the fundamental concepts involved in human movement study. An introduction to the ACL, its structure and its deformation behavior is made. Following that, a method of modeling the human body as a system of multiple rigid body segments is explained, as are methods of acquiring human movement data. Finally, methods of dynamic simulation with a complete musculoskeletal model, comprised of both rigid body segments and soft tissues (muscles, tendons, ligaments), are explained.

\subsection{Kinesiology}

Kinesiology is a science also known as Human Kinetics, and involves the analysis of human movement. It focuses on how the body functions and moves. The Kinesiologist assesses bodily movements and performance in the areas of sports, recreation, work environment and other daily activities. Prevention of injuries to ligaments like the ACL is a major challenge faced in modern day kinesiology. The important aspects in kinesiology, required to study the ACL are discussed in the following sections. 


\subsection{Anterior Cruciate Ligament (ACL)}

The anterior cruciate ligament is one of the four major ligaments of the knee as shown in

Figure 2. It is an articulating ligament (a moveable ligament that aids joint motion) of the knee joint that connects the inter-condyloidic eminence of the tibia to the lateral condyle of the femur. The ACL's main function is preventing excessive twisting and forward translation of the tibia in relation to the femur. Second only to ligaments of the ankle (usually posterior ones like the anterior talo-fibular), the ACL bears some of the highest loads during activities involving lower extremities ${ }^{42}$. While Figure 2 shows the location of the ACL in the knee, Figure 3 shows the musculature that surround and articulate the knee joint.

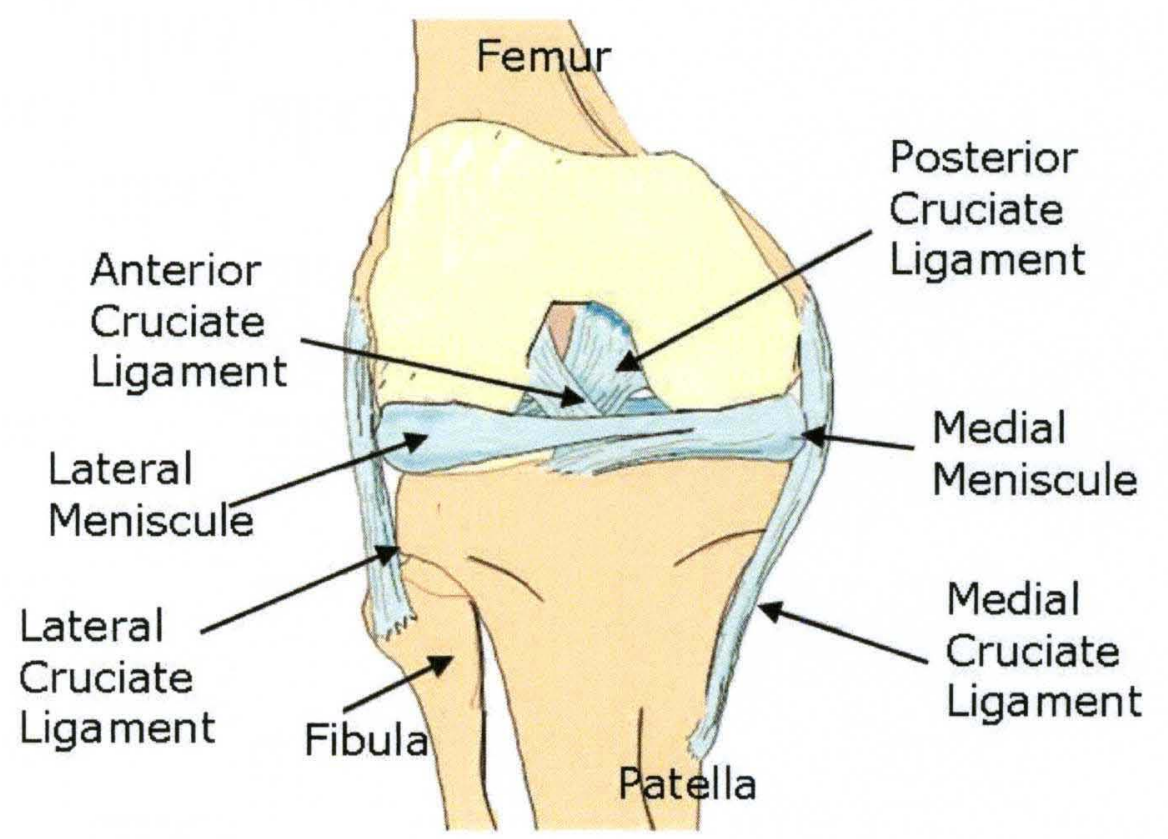

Figure 2. Location of main ligaments and bones of the knee. Top surface of tibia showing where both anterior cruciate ligament (ACL) and posterior cruciate ligament (PCL) attach. 


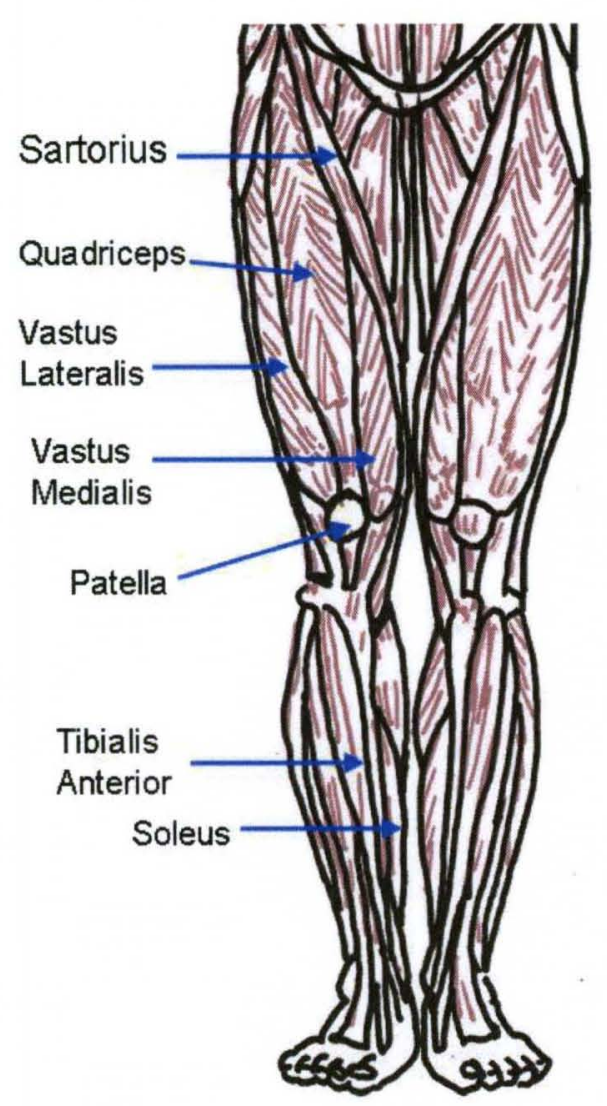

a

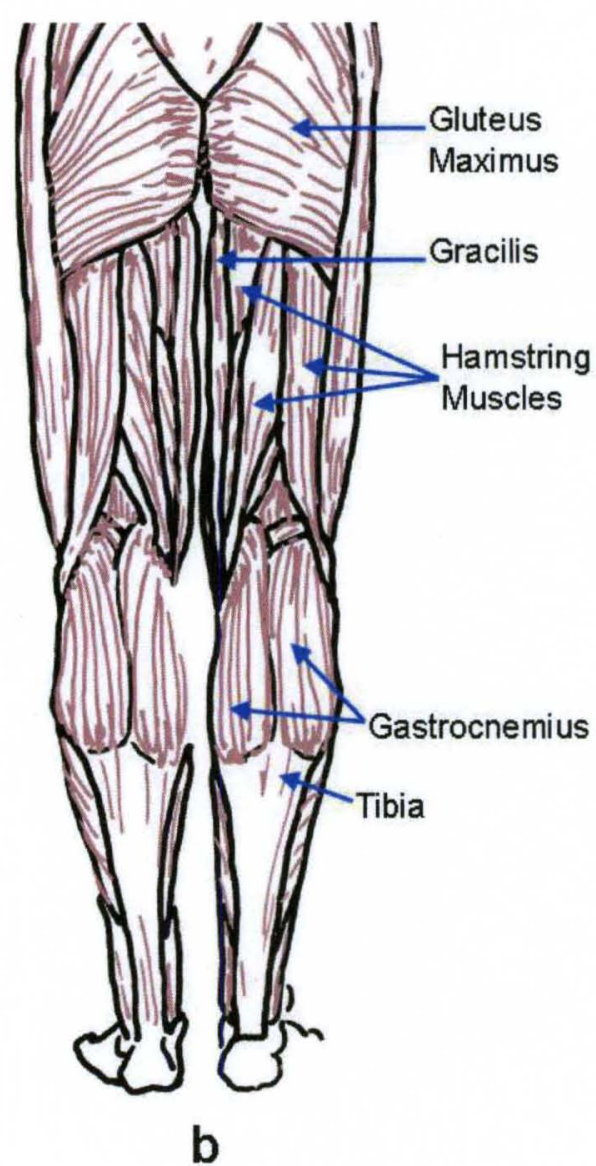

Figure 3. [a]: Front of knee with the quadriceps muscles. [b]: Back of knee with the gastrocnemius muscles and the hamstring muscle.

\subsection{ACL Structure and Mechanics}

Ligaments (like tendons) are generally composed of Type I collagen fibrils, a proteoglycan matrix and fibroblasts (cells) arranged in parallel rows. Ligaments generally consist of hierarchical arrangement of substructures as shown in Figure 4[a]. The structural characteristic at the fibril level plays the most significant role in the mechanics of ligaments. In a non-stretched ligament the fibrils are crimped, the crimp of the fibril unfolds as the ligament lengthens. This ability to crimp makes the ligament deform in a non-linear way when progressively loaded as Kastelic et al., 1978, points out ${ }^{40}$. The 
non-linear behavior is shown in Figure $4[\mathrm{~b}]$. In the beginning at the 'toe' region, the crimp of the collagen fibrils are stretching out and relatively low stiffness occurs in the first two regions. After most collagen fibrils straighten out, the collagen fibril backbone itself starts stretching, giving rise to higher material stiffness 9,40 . In the third stage individual fibrils within the ligament begin to fail contributing to accumulated damage, which finally ruptures the complete structure.
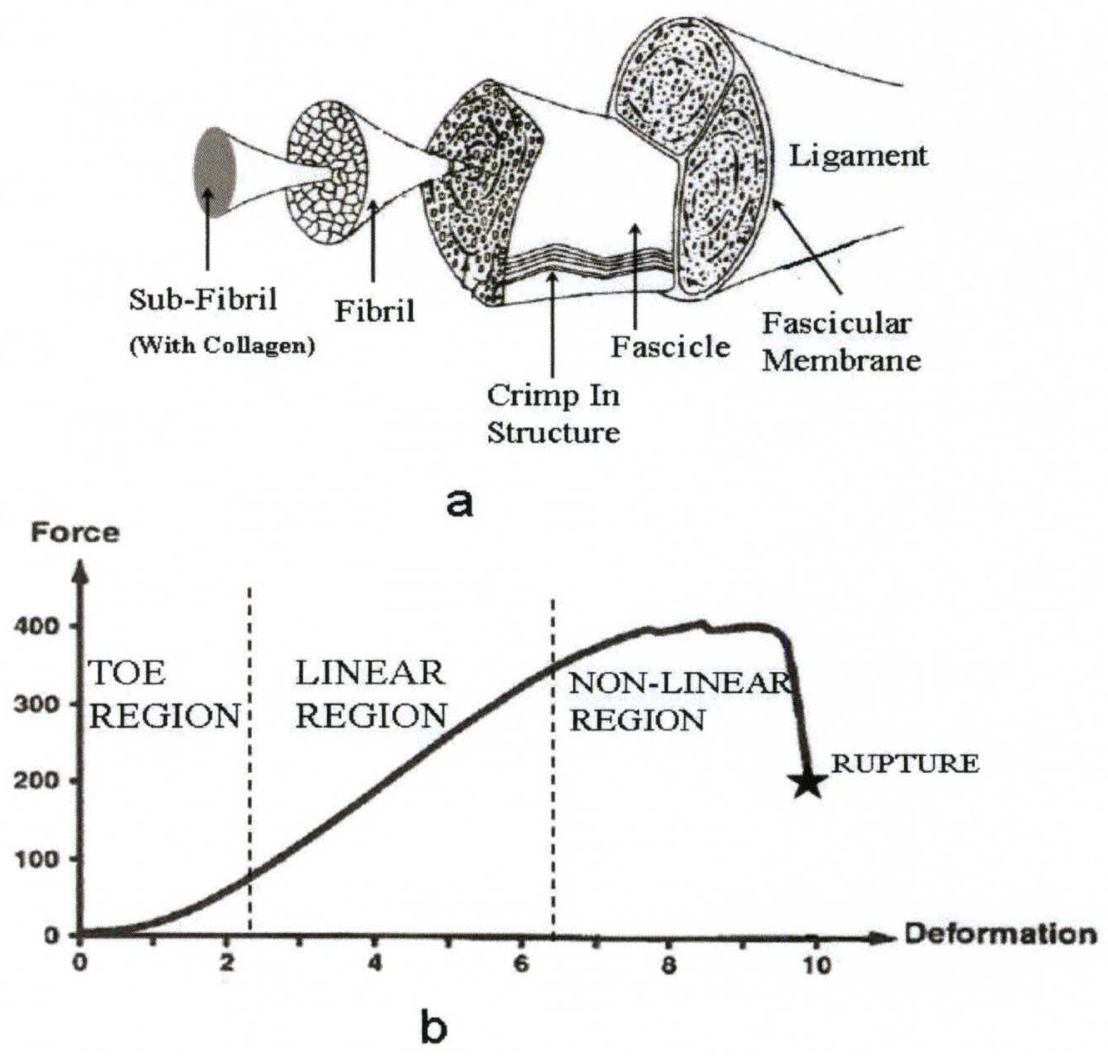

Figure 4. [a]. Ligament hierarchical structure complete with fibrils and collagen showing the crimp in fascicles or fibril bundles. [b]. Load deformation curve showing the nonlinear behavior from an in-vitro rabbit ligament experiment.

Source. http.//www.engin.umich.edu/class/bme456/ligten/ligten.htm 


\subsection{Characteristics of ACL Injury}

The ACL is most commonly damaged in noncontact sporting maneuvers such as sprinting, side-cutting and jumping (Section 1). For example when someone tries to rapidly change direction with the leading leg out, the ACL is either fully or partially

extended ${ }^{33,47,58}$ to counteract twisting of the knee. If the knee is locked, and the leg is firmly planted, the ACL is most likely to be strained to the point of yielding.

The study primarily focuses on the three most common factors that influence ACL loads during the execution of a drop-jump, as mentioned in Chapter 1. These factors include decreased knee flexion, increased knee valgus and decreased knee flexor to extensor muscle recruitment and are:

Low magnitude of knee flexion at high loads. This causes high moments and compressive forces in the knee. The combination results in higher energy being absorbed by the knee.

Large angle of valgus of the knee. This causes outward motion of the lower leg away from the medial plane. With valgus, there generally is extreme abduction of the lower leg accompanied by extreme eversion of the foot where both lower leg and foot move away from the body's medial plane. As McLean et sl., 2005, pointed out only sagittal plane knee motion does not explain ACL injury ${ }^{44}$. 
Low knee flexor/extensor recruitment ratio. This implies that the activations of the knee flexor muscles are low compared to those of the extensor muscles. More often conditions such as neurological imbalances cause low activation in the flexor muscles. If at the same time the extensor muscles have normal function, an imbalance of torques happen at the knee joint affecting both ACL and PCL.

\subsection{Kinematics}

Kinematics is the study of motion of particle(s) and their time derivates, velocity and acceleration. In human movement, segments and joints are considered a system of particles that form a rigid body and undergo both translational and rotational motion ${ }^{43,66 \text {, }}$ 76, 77 . The rule that all particles in a rigid body undergo the same rotational motion is an important feature of rigid body kinematics. Anatomical kinematic data is presented according to a right handed (RH) reference coordinate system called the global coordinate system as shown in Figure 5[a]. In the RH system the direction of positive rotation is anticlockwise around the positive axis normal to the plane of rotation. Figure $5[\mathrm{~b}]$ shows the directions of rotation of individual segments at the joints mostly in the Sagittal plane, even though other directions are possible to some extent. 


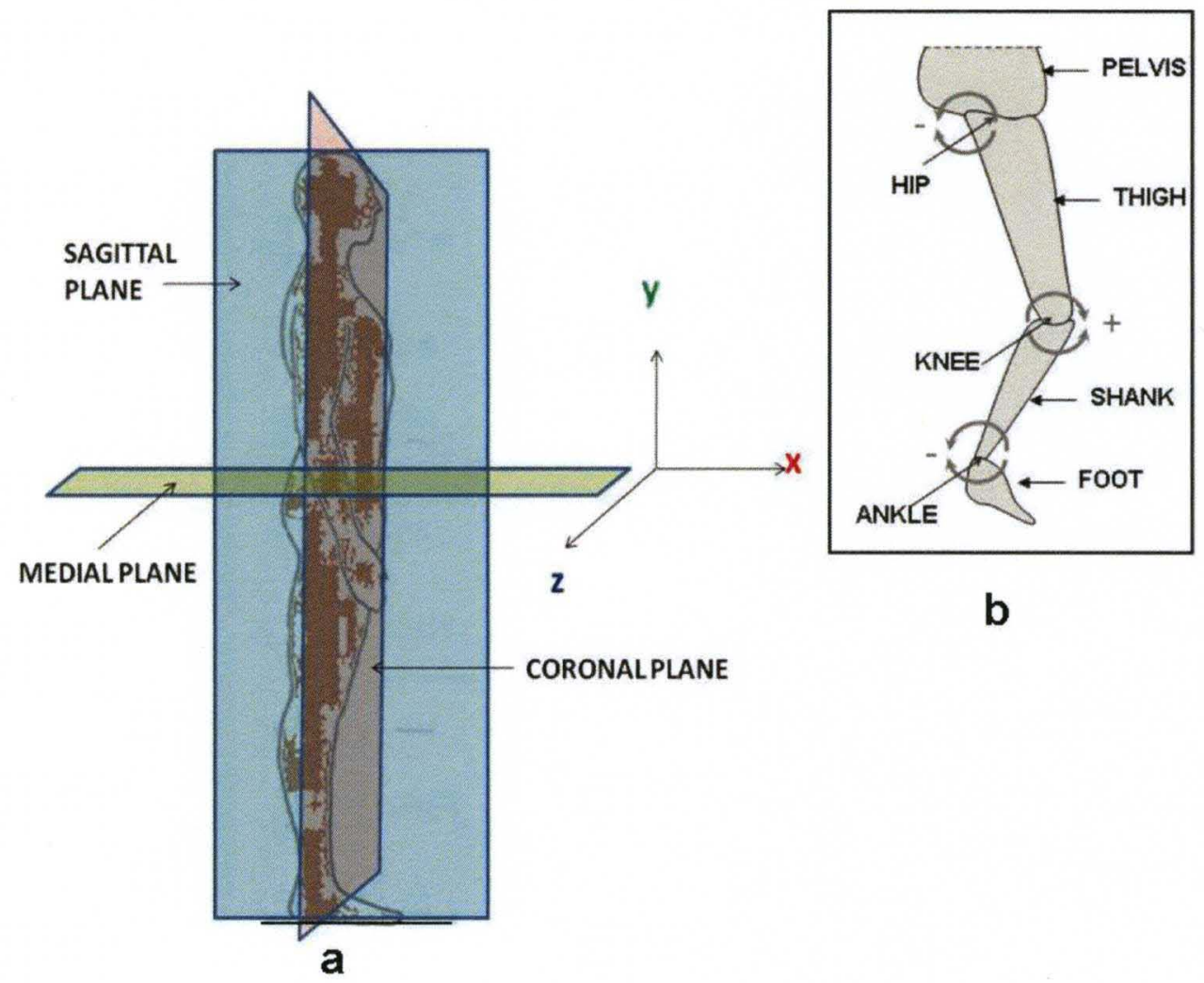

Figure 5. [a]: Orientation of the global reference coordinates system (GCS) with main directions of translation and main planes of rotation. [b]: Moments at the three main joints of the lower body. The moment directions depend on the direction of extension of the distal segment.

\subsection{Human Body Model}

To recreate ACL injury within a simulation environment, the first requirement was to develop an anatomical model of the human lower extremities. Modeling the lower extremities, the left and right legs, involve dividing each leg into three distinct segments. the thigh, the shank and the foot and its comprehensive details are given in Winter, 1990, also shown in Figure $6^{76}$. Description of the abbreviated labels used in Figure 6 is given in Appendix D. 


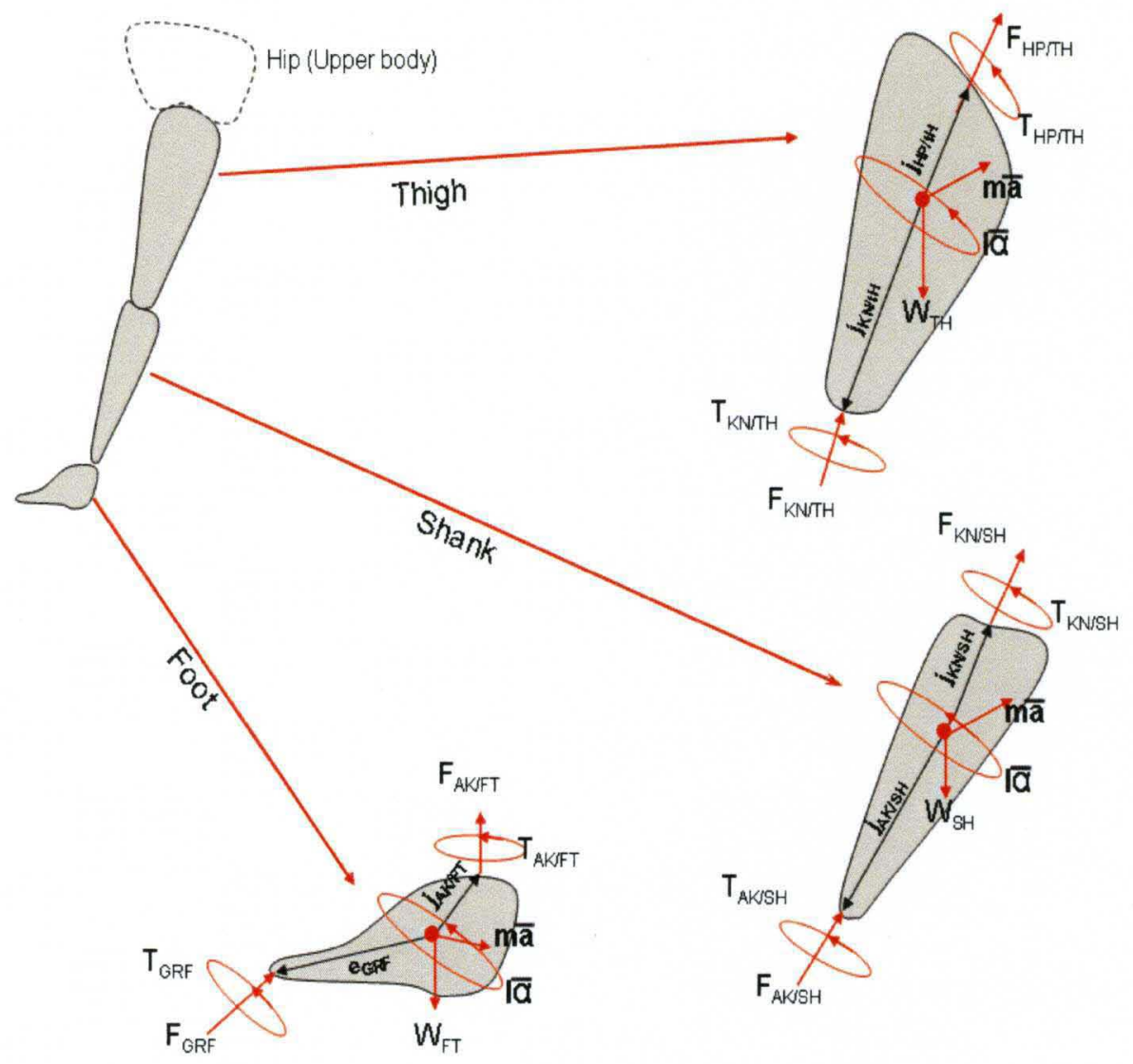

Figure 6. Three main segments of the lower extremities. Forces and moments are named according to the joint and segment names where they act. $\mathrm{F}_{\mathrm{GRF}}$ implies ground reaction force acting on the foot when it is in contact with the ground.

See Appendix D for a description of terms.

The upper body model that includes the torso, shoulders, back, head and arms have been left out of this discussion. The three segments per leg are linked segments and are held together by the hip, knee and ankle joints. The joints function to cause relative motion between the segments and also constrain such motion mostly (but not always) to rotation in a single plane. Forces and moments acting at each joint are named according to the joints and segments where they act. For example, the force acting on the ankle joint attached to the foot is termed $\mathrm{F}_{\mathrm{AK} / \mathrm{FT}}$ while the force acting on the ankle joint attached to 
the shank is termed $\mathrm{F}_{\mathrm{AK} / \mathrm{SH}}$. The weight of each segment at its center of mass (COM) is defined by the segment name, such as $\mathrm{W}_{\mathrm{FT}}, \mathrm{W}_{\mathrm{SH}}$ and $\mathrm{W}_{\mathrm{TH}}$. The ground reaction force $\left(\mathrm{F}_{\mathrm{GRF}}\right)$ and moment $\left(\mathrm{T}_{\mathrm{GRF}}\right)$ act on the foot segment only when there is transmission of force and moment between the foot and the ground during ground contact activities such as walking, running, and jumping.

Several important assumptions apply to the dynamic model of the lower extremities ${ }^{10,23,76}$ which are,

1. All segments are considered rigid bodies.

2. Each segment consists of a group of muscles and bones of constant mass all attached to hinged joints at the two ends of the segment.

3. The location of the COM for each segment, calculated from anthropometric data, remain fixed.

4. The length of the segment, defined by its longest bone, remains unchanged in motion. During activation, muscle length changes do not cause changes in the segment length.

5. The mass moment of inertia about the segment COM remains constant at all times during motion.

\subsection{Joint and Segment Kinematics}

The two segments connecting at a joint are called distal and proximal segments with the distal segment being closest to the ground. Joint motion is perceived as the motion of the distal segment relative to the proximal one. As the body moves, the relative positions 
between the two rigid body segments attached at the common joint change. With such motion segments and joints both translate and rotate.

One of the key features in joint kinematics is the orientation angles between the distal and the proximal segments. During motion, aside from translation, the change in orientation between the two segments or the orientation of a segment relative to its initial position is seen as a series of rotations referenced to a three dimensional co-ordinate system. The new orientation is quantified as the result of these sequential rotations about the three coordinate axes. Figure 7 shows the orientation of the unchanged reference frame and the three planes of rotations, and the final changed reference frame.
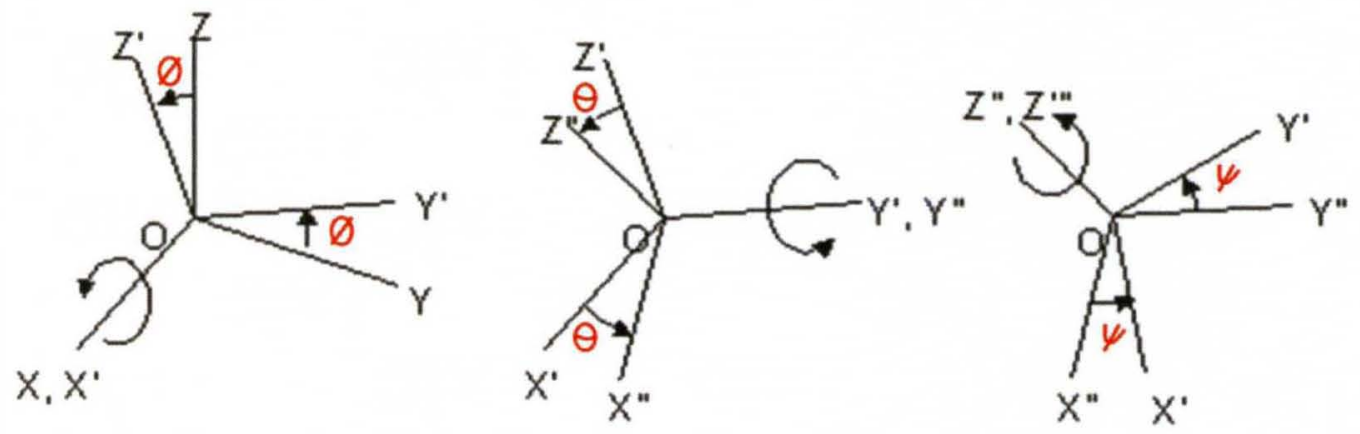

Figure 7. Transformation from the $[\mathrm{x} y \mathrm{z}]$ system to the $\left[\mathrm{x}^{\prime \prime \prime} \mathrm{y}^{\prime \prime \prime} \mathrm{z}^{\prime \prime \prime}\right]$ system using the Cardan system of rotation.

Out of several 3D sequences, the Cardan sequence of rotation is the most widely used in human movement analysis ${ }^{66,76,77}$. In a common Cardan sequence the first rotation is in the $y-z$ plane (flexion-extension). The second is in the $x-z$ plane (abduction/adduction). The third and final rotation happens in the $x-y$ plane (internal/external) rotation. Details 
on deriving the rotation matrix are given in Winter et al, $1990^{32}$. The Cardan sequence is most suitable for the purposes of this study since its planes of rotation are aligned with the usual human body planes (Figure $5 \mathrm{a}$ ). The $3 \times 3$ rotation matrix using the three angles of rotation is given by

$$
\left[\begin{array}{l}
X \\
Y^{-} \\
Z
\end{array}\right]=\left[\begin{array}{ccc}
c\left(\theta_{j}\right) c\left(\psi_{j}\right) & s\left(\varphi_{j}\right) s\left(\theta_{j}\right) c\left(\psi_{j}\right)+c\left(\varphi_{j}\right) c\left(\psi_{j}\right) & -c\left(\varphi_{j}\right) s\left(\theta_{j}\right) c\left(\psi_{j}\right)+s\left(\varphi_{j}\right) s\left(\psi_{j}\right) \\
-c\left(\theta_{j}\right) s\left(\psi_{j}\right) & -s\left(\varphi_{j}\right) s\left(\theta_{j}\right) s\left(\psi_{j}\right)+c\left(\varphi_{j}\right) c\left(\psi_{j}\right) & c\left(\varphi_{j}\right) s\left(\theta_{j}\right) s\left(\psi_{j}\right)+s\left(\varphi_{j}\right) c\left(\psi_{j}\right) \\
s\left(\theta_{j}\right) & -s\left(\varphi_{j}\right) c\left(\theta_{j}\right) & c\left(\varphi_{j}\right) c\left(\theta_{j}\right)
\end{array}\right]\left[\begin{array}{l}
X \\
Y \\
Z
\end{array}\right]
$$

where the proximal $\left[\begin{array}{lll}x^{\prime} & y^{\prime} z^{\prime}\end{array}\right]$ coordinate system is rotated into the distal $\left[x^{\prime \prime \prime} y^{\prime \prime \prime} z^{\prime \prime}\right]$ coordinate system.

\subsection{Collection of three Dimensional Kinematic Data}

A common method of acquiring three dimensional kinematic data is by using a multicamera setup. Using two dimensional position data captured by each camera and a camera calibration matrix, the planar image data is reconstructed into three dimensional spatial data. Several reflective markers are placed at strategic points on the subject under study. When a marker is detected by a minimum of two cameras, the digitized positional data are sent to a data acquisition system, where they are then reconstructed into spatial coordinates.

Shapiro et al. in $19788^{46}$, Dapena et al. in $1982^{14}$ and Hollister, et al., $1993^{38}$ are among the pioneers who developed the technique for three dimensional reconstruction from two dimensional cine images. A brief description of this reconstruction technique called Direct Linear Transformation (DLT) method is given in the following paragraph. 
The purpose of DLT is to convert two dimensional image plane data (from the cameras) into three dimensional objects space data by the use of a three dimensional conversion matrix. The matrix, which contains eleven independent parameters, is constructed using a minimum set of six non co-linear points identified by the cameras. Naturally, all control points have pre-determined locations in three dimensions. When more than six points are used, the redundant points are incorporated into the DLT calculations by using a least squares method. Once DLT parameters are determined, positions of markers placed on subjects are ready to be converted into three dimensional data. In 1988 Hatze et al. proposed a high precision method of three dimensional object space reconstruction called the Modified DLT method ${ }^{32}$. The motion acquisition application, Evart ${ }^{\mathrm{TM}} 5.0$, made by Motion Analysis Corporation, Santa Rosa, CA, used for this study utilizes the modified DLT method.

\subsection{Kinetics}

Fundamental to the analysis of human dynamic systems are the computations of forces, moments, energy flows, stresses and strains. Katz et al., 1939, followed by Spoor et al., 1980 and Winter et al., 1990 were among the first to develop methods for computing rigid body dynamics for the human body ${ }^{10,41,66,75,76,77}$. These kinetic data are generally computed at the segment COM and the joints. At a particular segment COM, the net force and moment are considered internal. In addition, external forces like ground reaction forces and segment weight as well as external moments are applied at the joints or some part of the segment. For the joints, the net force and moment are the summation of forces and moments applied by muscles, ligaments and tendons in the distal and 
proximal segments as well as external forces such as gravitational and ground reaction forces. Following the computation of the net force, other kinetic data like moments, energy, power, stresses and strains may be computed ${ }^{35,36}$.

All kinetic data, including forces, are presented according to the GCS shown in Figure $5[\mathrm{a}]$. Figure $5[\mathrm{~b}]$ shows the convention for moment direction at each joint. The direction of moment on a joint is defined by the direction of extensor moment acting on its distal segment. For example the clockwise moment direction of the ankle joint is according to the extensor moment acting on its distal segment, the feet.

It is of vital importance to correctly process external and internal forces and moments acting on a rigid body. In order to estimate the net force acting on a segment COM, one must correctly estimate all external forces acting on the segment. External forces like weight are estimated using body measurements, weight of subject and anthropometric tables. Other external forces like the GRF is estimated with the help of transducers placed on the ground. The method of measuring GRF is detailed in the following section.

\subsection{Ground Reaction Force}

For a rigid body under non-contact motion like walking, running and jumping there are only two main sources of external forces. One is the body weight and the second is the ground reaction force in interaction with the ground which comes in contact with the

foot $41,66,75,76,77$. In the laboratory, GRF is normally measured by a force-plate device composed of two metallic plates coupled together with transducers as shown in Figure 8. 
The figure shows a (strain gage based) force plate from Bertec Corporation, Columbus,

Ohio, used in this study. It was considered that the force plate's local coordinate system $\left(x_{f} y_{f} Z_{f}\right)$ did not coincide with the laboratory or global coordinate system $\left(X_{L} Y_{L} Z_{L}\right)$. Hence a transformation matrix was used to orient the forces and moments with the second global system. The transformation matrix was both rotational and translational. The rotational matrix consisted of a $3 \mathrm{X} 3$ matrix with components $\theta i j$ where $i=1 . .3$ and $j=1 . .3$. The translational vector $\boldsymbol{r}=\left\{r_{1} r_{2} r_{3}\right\}$ consisted of the components of displacement between the two coordinate systems.

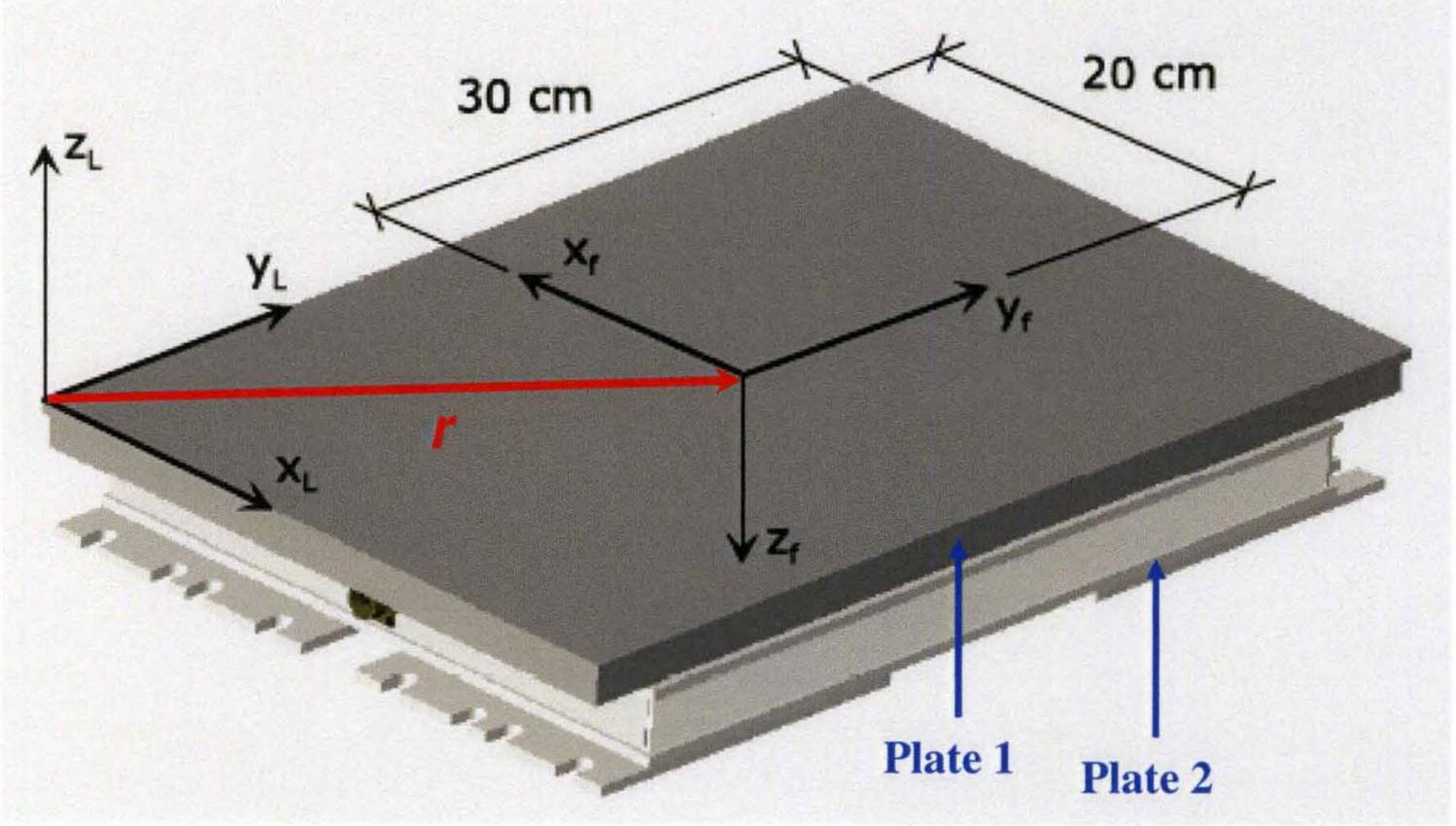

Figure 8. Typical force plate showing the force plate's local coordinate system and the motion analysis global coordinate system. The ground reaction load is measured in the force plate's local coordinate system denoted by the subscript " $\mathrm{f}$ ". Then the components of the force and moment vectors are transferred to the laboratory coordinate system indicated by the subscript " $L$ ".

Source. bertec.com/uploads/pdfs/manuals/ForcePlateManual.pdf. 
This force-plate has embedded strain gages between its two plates (not shown in Figure 8) that reflect the force acting between the foot and the ground. The feedback from the gages gave the ground reaction force and moment at the center of pressure. If multiple force plates are used, the resultant ground reaction force and moment of force about the global origin at the combined center of pressure need to be obtained.

\subsection{Inverse Dynamics}

Inverse dynamics is the process by which kinematic data and external force data (e.g., force plate) are used for calculating kinetic variables at a given anatomical location such as a joint or segment COM. Inverse dynamics also requires anthropometric data matched to individual subjects so that center of mass (COM) and moment of inertia (MOI) are correctly determined for body segments.

Using the orientation angles given in Section 2.5, all known kinematic and kinetic data are first converted to an appropriate fixed coordinate system called the anatomical reference system. The known quantities are segment center, moment of inertia, distal forces and moments. The only unknowns are the proximal forces and moments which are obtained from a set of equations combining the known and unknown data. A brief description of how the knee joint data is calculated using a chain of equations is given below. It begins with equations for Newton's laws of motion for a system of forces and moments given by

$$
\sum \bar{F}=m \tilde{a} \quad \sum \bar{M}=(\dot{H})
$$


Where $\sum \bar{F}$ is net force acting on the system,

$m \tilde{a}$ is mass times system acceleration,

$\sum M$ is the net moment acting on the system, and

$\dot{H}$ is rate of change in angular momentum.

The foot is the first segment for which the unknown kinetic quantities are solved. For the foot segment in Figure 6, the balance of forces and moments are given are given by

$$
\begin{aligned}
& \sum \bar{F}_{F T}=F_{G R F}+F_{A K / F T}+W_{F T}=m \tilde{a}_{F T} \\
& \sum \bar{M}_{F T}=e_{G R F} F_{G R F}+j_{A K / F T} F_{A K / F T}+T_{G R F}=\dot{H}_{F T}
\end{aligned}
$$

Moving on to the shank segment, the balance of forces and moments are given by

$$
\begin{aligned}
& \sum \bar{F}_{S H}=F_{K N / S H}+F_{A K / S H}+W_{S H}=m \tilde{a}_{S H} \\
& \sum \bar{M}_{S H}=j_{K N / S H} F_{K N / S H}+T_{K N / S H}+j_{A K / S H} F_{A K / S H}+T_{A K / S H}=\dot{H}_{S H}
\end{aligned}
$$

Descriptions of all terms used in equations 3-6, as well as in Figure 6 are given in Appendix D. Following equations (5) and (6), the joint force and joint moment at the knee are now to be calculated. Calculations of knee power and energy absorbed are also possible ${ }^{75}$. Knee power is the dot product of knee torque and angular velocity given by

$$
P_{K N}=T_{K N} \cdot \omega_{K N}=T_{X, K N} \cdot \omega_{X, K N}+T_{Y, K N} \cdot \omega_{Y, K N}+T_{Z, K N} \cdot \omega_{Z, K N}
$$


Again, a description of terms in the equations are given in Appendix D. Energy absorbed and generated by the knee is then calculated by knee power integrated over time. However all freshly calculated force and moment data are still in the anatomical local reference system and need to converted back into global reference system ${ }^{66,}{ }^{76}$.

\subsection{Electromyography}

Electromyography (EMG) is a method for detecting the activation of muscles when

electrical potentials are sent from the peripheral neurons into the muscle cells ${ }^{13,14,22}$. Muscle tissue at rest is normally electrically inactive, with the exception of the area of the synapse-muscle junction, which is generally electrically active. There are two kinds of EMG. The first type is non-invasive surface (skin) mounted and the second is an intramuscular (inserted) type. When a muscle is voluntarily contracted, action potential starts developing. As the action potential rises, the strength of the muscle contraction further increases.

For the purposes of this study, surface electrodes were used. Further detail on electrode placement and EMG data acquisition, filtering and smoothing is given in Chapter 3.

\subsection{EMG Data Filtering}

Butterworth filters are some of the most commonly used digital filters in motion analysis, mainly because they can precisely control the frequency components of signals. Digital Butterworth filters vary between low-pass, high pass, and band-pass. The band-pass type of filter is especially useful since the random errors at very high and low frequencies, in 
raw position data, are rejected. In addition, a notch Butterworth filter assures that all common building electrical noise between $60-62 \mathrm{~Hz}$ is rejected.

The gain $\mathrm{G}(\omega)$ of an $n$-order Butterworth low pass filter, given in terms of the transfer function $\mathrm{H}(\mathrm{s})$, is given by

$G^{2}(\omega)=|H(j \omega)|^{2}=\frac{G_{0}^{2}}{1+\left(\frac{\omega}{\omega_{c}}\right)^{2 n}}$

where $\mathrm{n}=$ order of filter, $\omega_{\mathrm{c}}=$ cutoff frequency.

In general it is seen that as $n$ approaches infinity, the gain characteristic becomes a rectangular function and in a low-pass filter, frequencies below cutoff will be passed with gain $\mathrm{G}_{0}$, the $\mathrm{DC}$ gain, while frequencies above will be suppressed. For smaller values of $n$, the cutoff will be less sharp as shown in Figure 9. The working of a high-pass filter is similar but opposite and a band-pass filters is a combination of the two.

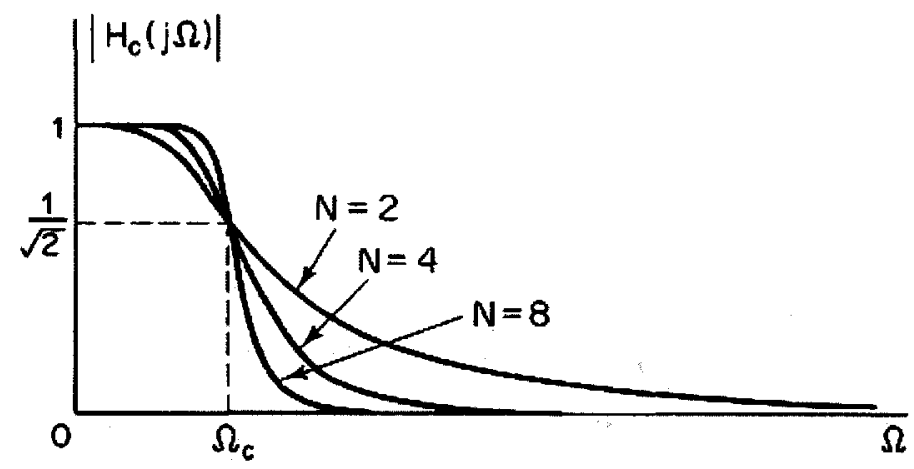

Figure 9. Low pass Butterworth filter showing gain $\mathrm{H}_{\mathrm{c}}$ at different poles of $\mathrm{N}=2,4$, and 8 . The cutoff frequency is given by $\Omega_{\mathrm{c}}$.

Source. Penheim, A.V., \& Schafer, R.W.. Discrete time signal processing. Englewood Cliffs, NJ. Prentice Hall. 1989. 


\subsection{Joint and Segment Position from Inverse Kinematics}

Once experimental data is collected, inverse dynamics is applied to obtain both kinematic and kinetic data including forces, moments and energies. Most authors present their data in the GCS system, co-incidental with the force plate axis system, a convention first used by Winter et al in $19833^{75}$.

The first step in Inverse Kinematics (IK) is calculating a set of joint angles and positions for the model that yields a motion trajectory from the experimental data. In each time step (frame) IK computes generalized coordinates and their derivatives which positions the model in a pose matching experimental (marker) position. These data, however, only describe joint kinematics. No details of connective tissue mechanics (muscle, ligaments and tendons) are available from IK. With IK the position, speed and acceleration for a knee joint at an instance in time are found but not the details of ACL mechanics.

\subsection{Musculoskeletal Model}

In order to analyze the ACL, a musculoskeletal model was required that included connective tissues like the ACL and other muscles surrounding the knee joint, subject to the same knee joint kinematics. A musculoskeletal model is used when the combined dynamics of bones, muscles and other bodily soft tissues, e.g., tendons and ligaments, produce optimal motion $5,7,11,15,16,17,19,21,25,28,68,69,71,72,73,78$. A musculoskeletal model composed of both rigid body segments (and joints) and soft tissue like ligaments, tendons and muscles was developed for this study. A complete body model was constructed, except for the arms, as shown in Figure 10. The model was developed in OpenSim 
(where generic prototypes already exist) $49,50,51,52,53,54,55,56,57,61$. The musculoskeletal model was composed of 13 body segments, 12 joints, 54 muscles, 23 rotational constraints, three translational constraints (actuators) and importantly, the left and right ACL. Muscle dynamics were computed from the Hill-Huxley ${ }^{37}$ model, consisting of both active and passive forces. The force-length-velocity relationship in muscle fibers is explained briefly in Appendix $B^{41}$. The right and left ACL was built from a Hill-Huxley model with non-existent active force. Figure 10 over the page also shows details of the lower extremity muscles and ACL.

\subsection{Static Scaling}

Scaling is performed based on the measured distances between marker locations and manually-specified scale factors. The marker locations are usually obtained from static trials. The non-scaled model has a set of virtual markers placed in the same anatomical locations as the experimental markers. The dimensions of each segment in the model are scaled so that the distances between the virtual markers match the distances between the experimental markers. Manual scale factors, which may come from other anthropometric analyses, can also used as an alternative to the measurement-based scaling for any type of body anatomy. 


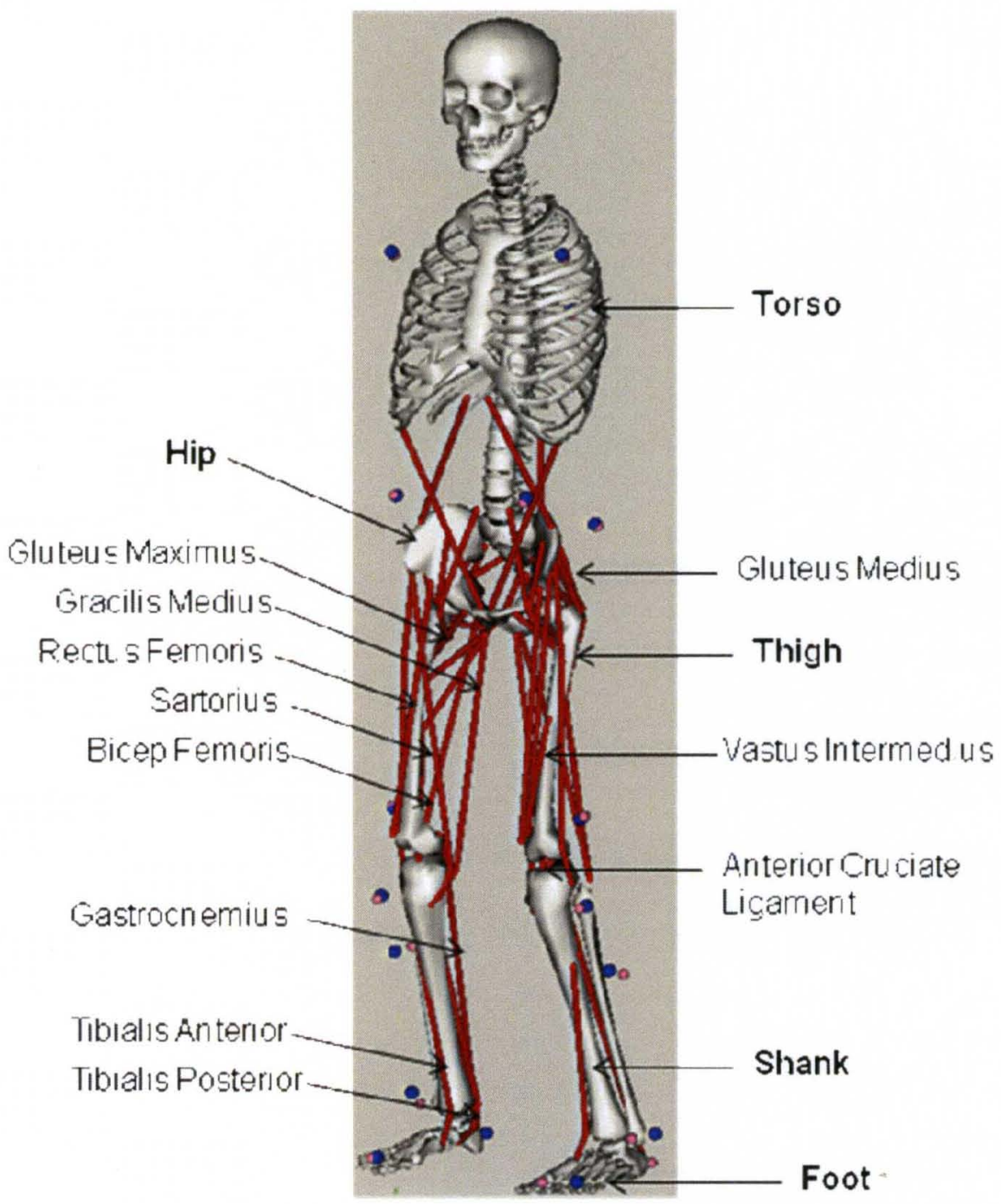

Figure 10. Musculoskeletal model showing main body segments (in bold) and muscles that contribute towards knee motion.

\subsection{Residual Reduction}

In some instances the overall force balance according to Newton's law (equation 3) becomes non ideal. In such a case, a residual force appears due to the unbalanced equation of forces. This error was rectified by adding a weight equivalent to the residual force to the segment where it occurred ${ }^{66,76}$. Residual Reduction (RR) is an optimization process for altering the torso center of mass for a dynamic model. This prevents biased 
leaning of the torso in the left-right and fore-aft directions. It also ensures the kinematics of the model to be more consistent with the ground reaction data. The equation for Residual Reduction is given by

$F_{x}+F_{\text {residual }}=m \tilde{a}_{x}$

$\mathrm{F}_{\text {residual }}=\mathrm{gc}_{\mathrm{c}} \cdot \mathrm{m}_{\text {additional }} \quad$ and $\quad \mathrm{m}_{\text {segment, } \mathrm{n}}=\mathrm{m}_{\text {additional }}+\mathrm{m}_{\text {segment, } \mathrm{n}-1}$

where $F_{X}$ is the force and $a_{x}$ is the acceleration acting in direction $x$,

$\mathrm{m}_{\text {segment, } \mathrm{n}}$ is the mass of segment $\mathrm{n}, \mathrm{m}_{\text {additional }}$ is the additional mass due to residual force.

In Residual Reduction, there are no muscles to apply forces to the skeleton. Instead, the model has torque actuators at each joint to apply forces to the various segments. For the purposes of this study, a whole body simulation with 23 actuators (1 degree of freedom per actuator) for 10 rigid body segments and 6 ground reaction forces is proposed. This way, a dynamic skeletal model is made to follow a prescribed motion.

\subsection{Computed Muscle Control (CMC)}

The basic concept of Computed Muscle Control (CMC) applies to most modern day machinery (with multiple components) whose performance must be controlled with the help of an integrated feedback system ${ }^{4,5}$. In human biomechanics, the purpose of CMC is to compute a set of muscle excitations (controls) that will drive a musculoskeletal model to perform a specific set of kinematics $1,2,3,4,16,72,73$. The first step in CMC is to compute a set of accelerations, $\bar{q}$, which drives the model coordinate toward the experimental coordinates, $\vec{q}_{\text {exp }}$. The desired accelerations are computed using a forwardly 
computed 5th order Runge-Kutta-Feldberg ${ }^{72}$ critically damped PD control law, whose equation is given by

$$
\ddot{\vec{q}}(t+T)=\ddot{\vec{q}}_{e x p}(t)+k_{v}\left(\dot{\vec{q}}_{e x p}(t)-\dot{\vec{q}}(t)\right)+k_{p}\left(\vec{q}_{e x p}(t)-\vec{q}(t)\right)
$$

where $\ddot{\vec{q}}, \dot{\vec{q}}$ and $\vec{q}$ are the model's position vectors and their derivatives, $k_{v}$ and $k_{p}$ are the velocity and positional gains chosen to make the motion critically damped.

The next step in CMC is computation of the individual muscle controls, $\mathrm{x}_{\mathrm{i}}$, also known as "excitations". This is achieved through an optimization process called Sequential Optimization Programming, SQP ${ }^{73}$. Using SQP, the load at every joint is distributed across (joint) muscles contracting in synergy. The optimization procedure is implemented once joint coordinates and their derivatives are computed (from equation 10). The equations for determining the joint forces and torques $15,16,17,27,29,73$ are given by

$$
\vec{M}(\vec{q}) \cdot \ddot{\vec{q}}+\bar{C}(\vec{q}, \dot{\vec{q}})+\bar{G}(\vec{q})=\vec{R}_{m}(\vec{q}) \cdot \vec{f}_{m}+\ddot{R}_{g r f}(\vec{q}) \cdot \vec{f}_{g r f}
$$

where $\vec{M}(\vec{q})$ is the segment mass matrix, $\vec{C}$ is the combined Coriolis and Centrifugal velocities, $\vec{G}(\vec{q})$ is the gravitational force and $\vec{R}_{m}(\vec{q}) \cdot \vec{f}_{m}+\vec{R}_{g r f}(\vec{q}) \cdot \vec{f}_{g r f} \quad$ are the combined muscle forces (per unit muscle length) and GRF forces, and

$\tau_{j}=\sum_{i}^{N} \vec{R}_{m i}\left(\vec{q}_{i}\right) \cdot \vec{f}_{m i}$

where $\vec{f}_{m i}$ is the muscle force, $\vec{R}_{m i}$ is the moment arm and $\tau_{j}$ is the joint torque. 
The method by which muscle forces contribute to the joint torque forms the core of the CMC optimization process. First the muscle forces are determined by excitationactivation based neuromuscular modeling. This model, predicting the shortening dynamics of muscles, is known as the Hill-Huxley model, more details of which is given in Appendix C. The linear Hill-Huxley model used for estimating contribution from each muscle is given by

$f_{m}^{*}=\left\{a_{m}^{*} \cdot f_{h}\left(l_{m}^{*}, i_{m}^{*}\right)+f_{\text {passive }}\left(l_{m}^{*}\right)\right\} \cos \left(\alpha_{m}^{*}\right)$

where

$f_{m}^{*}=$ Steady state muscle force after contraction equlibriates

$a_{m}^{*}=$ Muscle activation when fiber velocity equals musculotendon velocity

$f_{k}\left(l_{m}^{\star}, i_{m}^{*}\right)=$ Muscle force from force - length - velocity surface curve

$f_{\text {passive }}\left(l_{m}^{*}\right)=$ Muscle passive force

$\alpha_{m}^{*}=$ Pennation angle

The final force from each muscle is then calculated by minimizing a cost function ${ }^{16,17,18,72,73}$ equation given by

$$
J=\sum_{i=1}^{n x} x_{i}^{2}
$$

where $\mathrm{J}$ is a performance criterion and $\mathrm{x}_{\mathbf{i}}$ are muscle excitations. Equation 14 in turn is constrained by:

$$
\begin{aligned}
& \dot{a}=(x-a)\left(\frac{x}{t_{a c t}}-\frac{(1-x)}{t_{\text {derac }}}\right) \quad x \geq a \\
& \dot{a}=\frac{(x-a)}{t_{\text {deart }}} \quad x<a
\end{aligned}
$$


where $a$ and $\stackrel{\circ}{a}$ are muscle activation and it's derivative directly representing muscle fiber calcium concentrations ${ }^{16,73}, x$ is the excitation and $t_{a c t}=10 \mathrm{~ms}$ and $t_{\text {deact }}=40 \mathrm{~ms}$ are activation and deactivation times.

To reduce the performance criteria $\mathrm{J}$, two important constraints are applied, $0<x<1$ and $0<a<1$. CMC formulation is also known as Static Optimization because the performance criterion is satisfied at a given instant in time during a simulation. A schematic of the CMC process is shown in Figure 11.

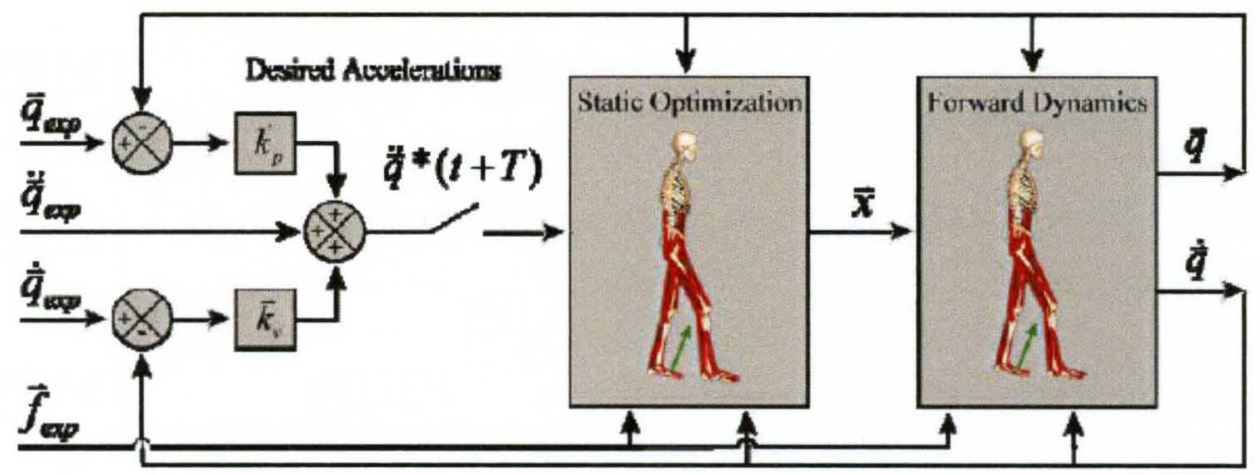

Figure 11. Schematic of close looped Computed Muscle Control (CMC) process and Forward Dynamic (FWD) process. Source: Thelen and Delp et al., 2003.

Sometimes due to inadequate forces supplied by the musculoskeletal models the entire joint torque is be supplied. To prevent the formulation from failing, a number of reserve actuators are added to a model that are able to make up for strength deficiencies in muscles. The reserve actuators have very low strength and therefore higher excitations are required to apply significant forces to the model. Since the optimization essentially reduces excitation, use of the (high excitation) reserve actuators is usually avoided. 


\subsection{Forward Dynamics Simulation (FWD)}

After muscle excitations are computed by CMC, Forward Dynamics (FWD) is used to drive a simulation where, for specific time intervals, detailed biomechanical data on muscle function are generated. The FWD uses the same model and actuator set used in $\mathrm{CMC}$, along with the initial states and controls computed during the CMC step. The goal of using FWD is to reproduce the same motion tracked by CMC and develop details of muscle biomechanics in a non-closed loop way. Joint states are found using a 5th order Runge-Kutta-Feldberg integrator along with the application of recorded actuator controls from $\mathrm{CMC}$ with no feedback or correction mechanisms. However the lack of feedback may causes the forward dynamic simulation to diverge from expected trajectory. This generally happen during longer simulations, where small differences accumulated over extended periods results in widely divergent trajectories.

The important benefit of FWD is that it allows the excitation of the ACL to be controlled. The ACL in this study is modeled as a fully passive tissue instead of the passive-active

Hill ${ }^{37,41}$ tissues of the other muscles. Hence for the ACL, excitation is ineffectual and may be completely shut down.

\subsection{Statistical Analysis}

Statistical analysis in this study was mainly for the purposes of establishing a relationship between important dynamic variables such as force-displacement and moment-angle correlations. Importantly, correlations were calculated for validating Forward Dynamics results against kinematics data from Inverse Kinematics for knee flexion and valgus 
angles. It was also used for generating the correlation between experimental EMG and muscle activation from simulation. Pearson product-moment correlation coefficient (r) was used to quantify the above correlations, where the general equation for Pearson correlation coefficient is given by

$$
r_{x y}=\frac{\sum_{i=1}^{n}\left(x_{i}-\bar{x}\right)\left(y_{i}-\bar{y}\right)}{\sqrt{\sum_{i=1}^{n}\left(x_{i}-\bar{x}\right)^{2} \sum_{i=1}^{n}\left(y_{i}-\bar{y}\right)^{2}}}
$$

where, $x_{i}$ and $y_{i}$ are the values for a series of $n$ measurements of $X$ and $Y, i=1,2, \ldots, n$ is the series index.

The sample correlation coefficient is then used to estimate the population Pearson correlation $\mathrm{r}$ between $\mathrm{X}$ and $\mathrm{Y}, \mathrm{r}_{\mathrm{xy}}$, as presented in Rodgers et al ${ }^{60}$. The Coefficient of determination $\left(\mathbf{r}^{2}\right)$ that measures the proportion of variability between data sets was also calculated by squaring the r-value, assuming linear relationship between the data sets. In addition, Pearson-p value, $95 \%$ confidence intervals were also calculated for the above dynamic variables. 


\section{CHAPTER III}

\section{EXPERIMENTAL METHOD AND ANALYSIS}

The process of motion capture involves multiple steps that are co-dependent on each other. Hence planning and executing the steps in a chronologically coordinated order is of paramount importance. Tasks like obtaining the written consent of the subjects, preparing subjects for trials, and conducting static strength tests must happen before any dynamic data are collected. During dynamic data collection, three dimensional kinematic data were generated using a high-speed, multiple camera motions capture system coupled to a 2-D to 3-D mapping process. Subjects performed drop-jumps from various heights ranging from $30-50 \mathrm{~cm}$ in steps of $10 \mathrm{~cm}$. The changes in platform height served as variable for increasing task difficulty and possible loading on the ACL. After obtaining dynamic data, further post processing involving calculation of trajectories and data smoothing using Evart's built-in Butterworth filters was performed. Finally, simulation of individual musculoskeletal models was performed and ACL loading and strains were analyzed. A flow chart showing the sequence of events is given in Figure 12 over the page.

Sections that follow outline the experimental setup and procedures for preparing the subjects for trial, data acquisition for static and dynamic strength tests, and finally details for post-processing and data analysis. 


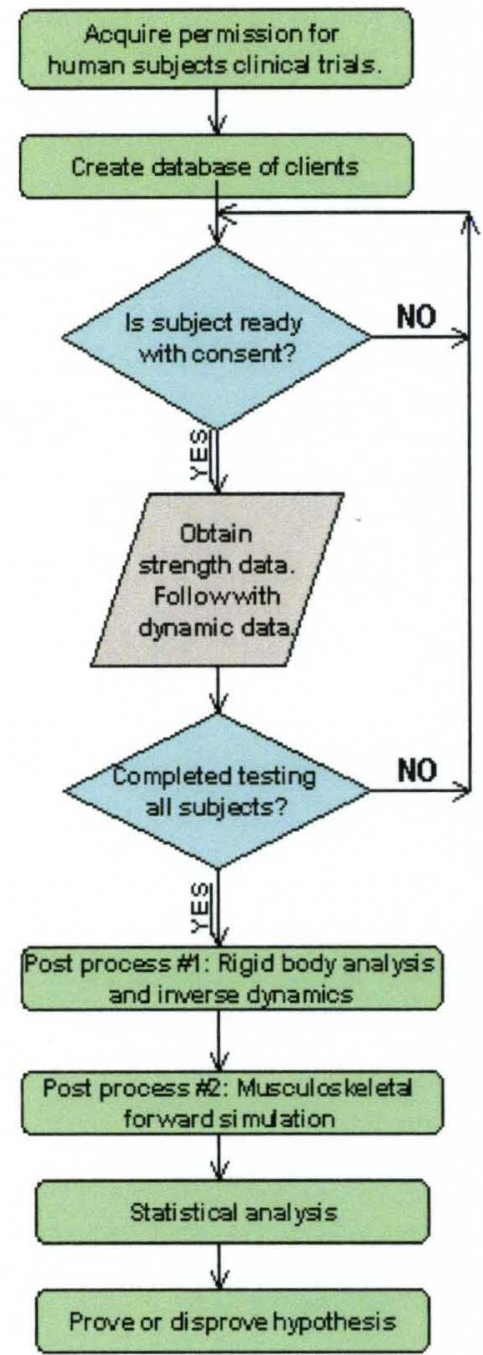

Figure 12. Flow chart of sequence of tasks for the project.

\subsection{Experimental Setup}

Drop jump trials were carried out on 13 subjects ( 2 without EMG). Motion analysis data were recorded using $8 \mathrm{Hawk}$ high speed cameras at $100 \mathrm{~Hz}$, sent through an eight channel hub into the real time Evart motion tracking system. Similarly, two six channel digital amplifiers sent force plate analog data to A/D convertors, which were then sent to the running motion capture system. Data was collected for both left and right feet on landing. 
The subjects, fitted with twenty reflective markers and eight EMG sensors each, conducted drop landings from three different elevations of $30 \mathrm{~cm}, 40 \mathrm{~cm}$ and $50 \mathrm{~cm}$. Kinematic data were edited and smoothed using built-in Butterworth filters in Evart prior to musculoskeletal analysis.

\subsection{Preparing the Subject}

Subjects recruited were healthy adolescent and young adult women who were capable of participating in drop-jump activities from a height of $50 \mathrm{~cm}$ or more. In the beginning each subject signed a consent form stating their intention to participate in the trials. For each subject, a number of basic anatomical measurements were then acquired. These measurements were body mass, height and knee (joint under study) width. The knee width was defined as the distance between the lateral and medial femoral condyles. The subjects were then fitted with twenty-four markers, as shown in Figure 13[a], in preparation for the static trial. Once the static trials were completed, the subjects were fitted with twenty markers, positions of which are in Figure 13[b]. They were also fitted with eight EMG sensors each on their legs, placed approximately on the left and quadriceps, vastus femoris, hamstring and gastrocnemius muscles, as shown in Figure 14. 


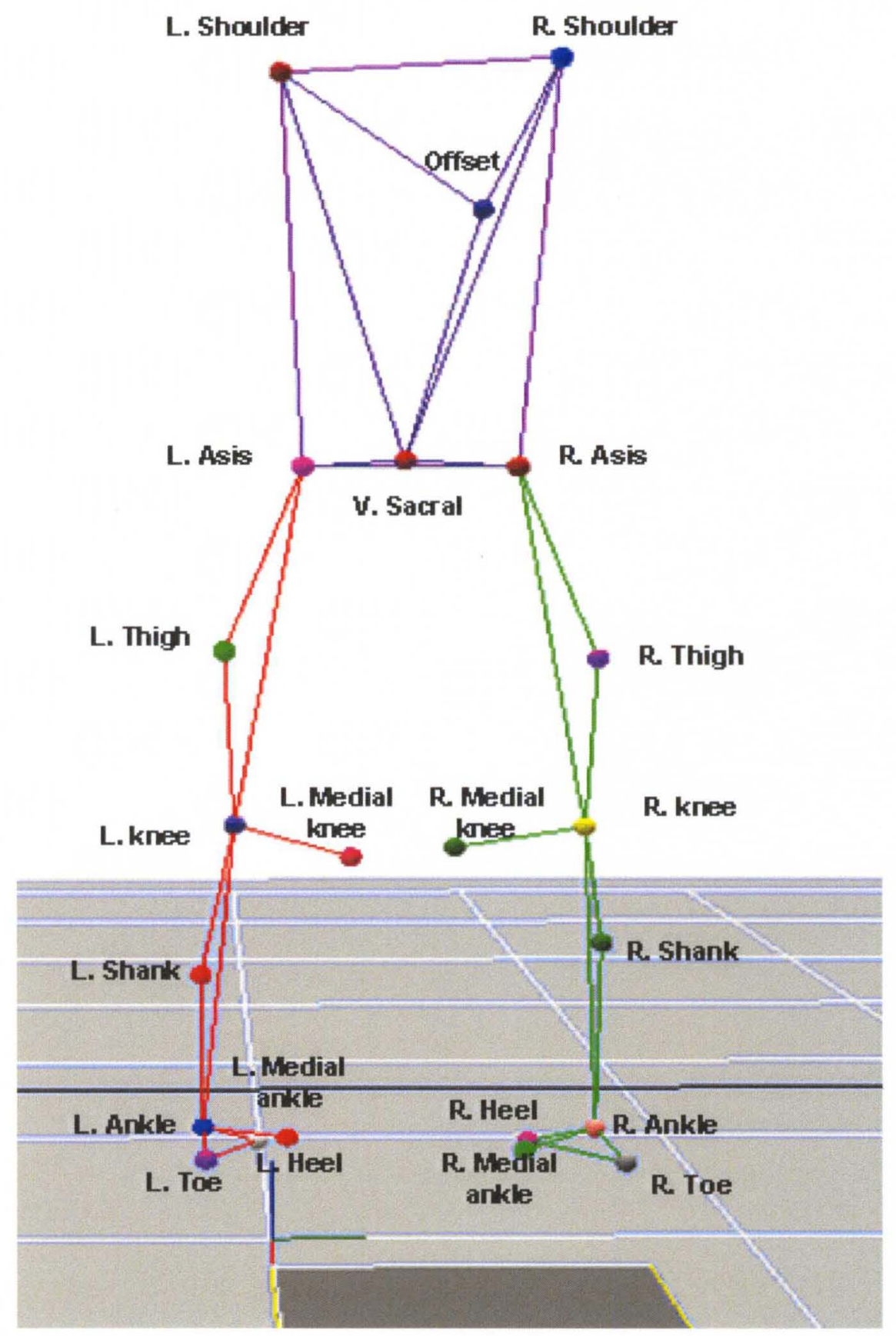

Figure 13. [a]. Marker placements on the whole body for static trials. The GCS coordinate axes is given by the triad of red, blue and green arrows at the left upper corner of the force plate. 


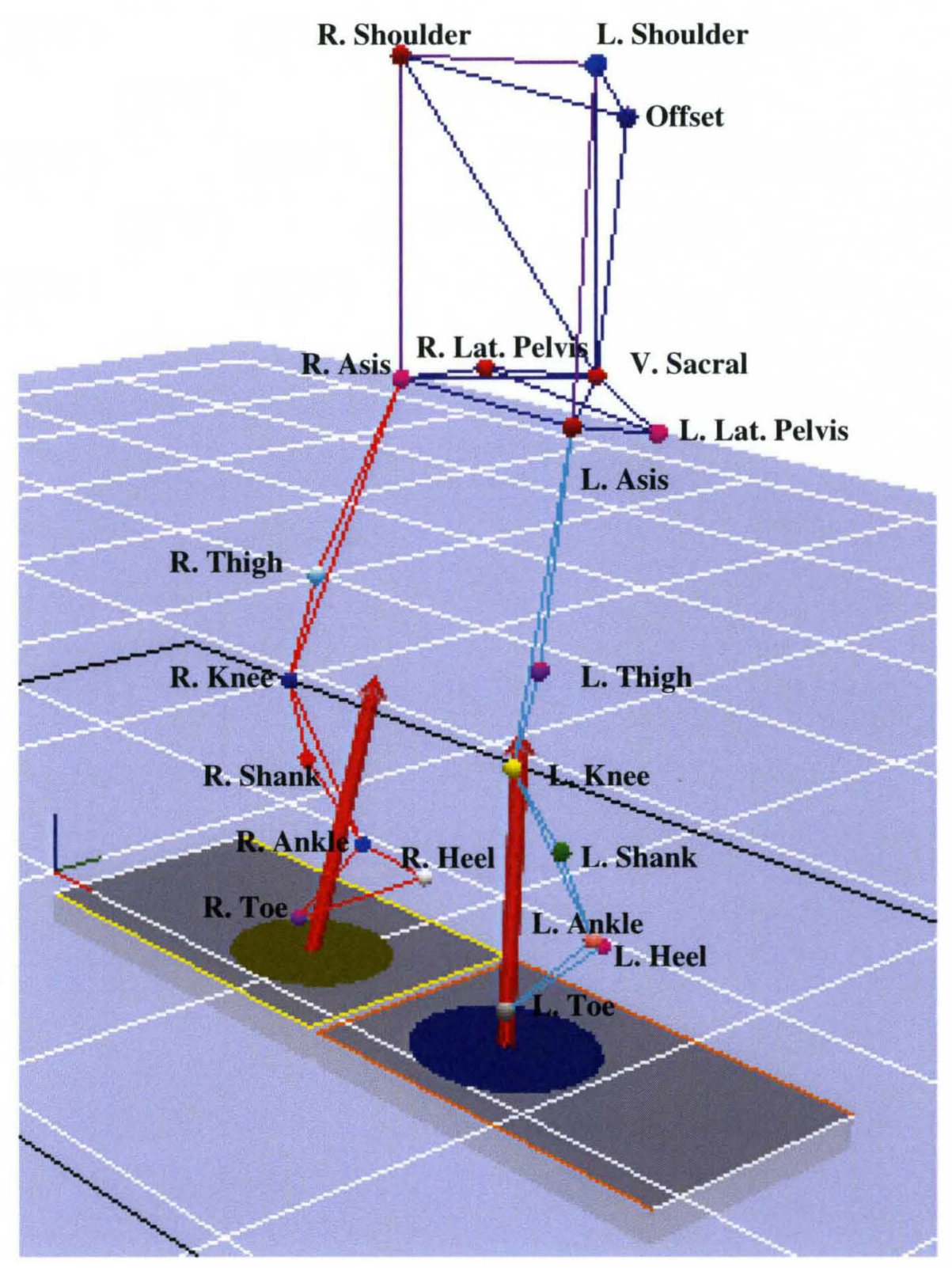

Figure 13. [b]. Marker placement on the whole body for dynamic trials. The red arrow shows the ground reaction force acting on the foot in contact with the ground. The two force plates are shown in grey. Circles on force plate indicate ground reaction moments. 


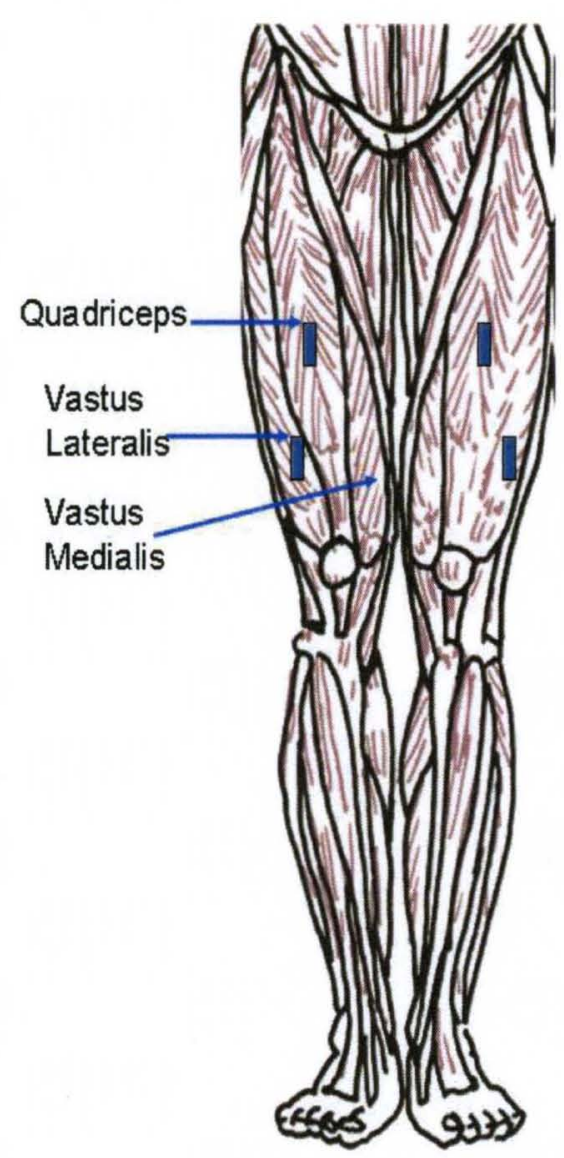

FRONT LOWER EXTREMITY MUSCLES

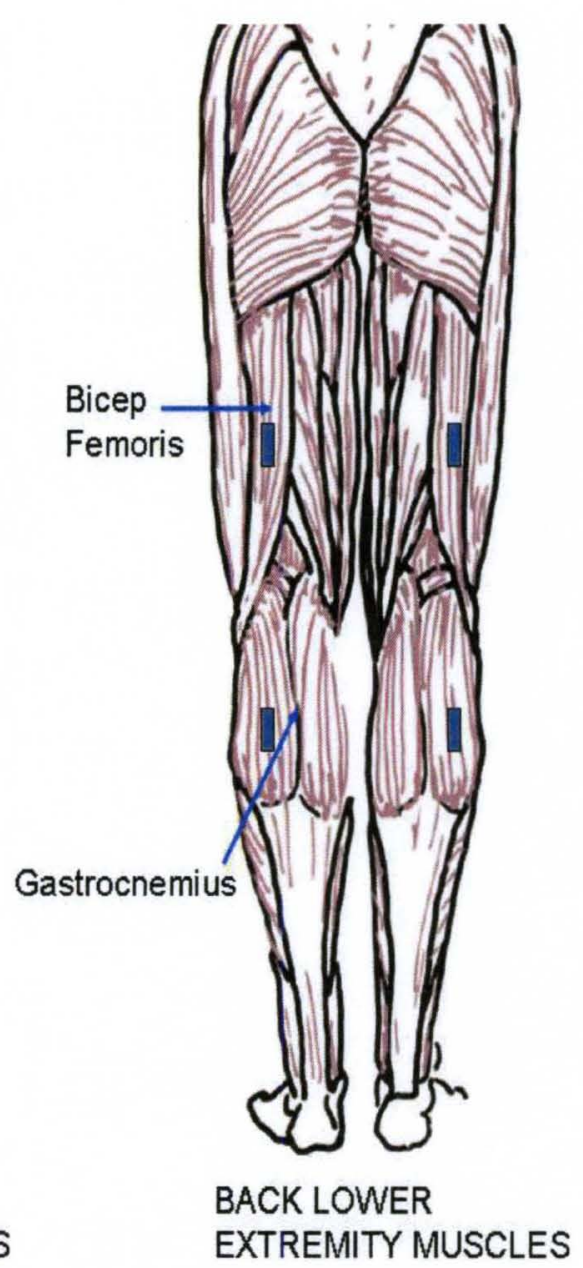

EXTREMITY MUSCLES

Figure 14. Placement of EMG sensors on muscles of the lower extremity. Front and back of the human leg muscular system showing the quadriceps femoris, vastus, bicep femoris and gastrocnemius muscles, used during this study.

\subsection{Isokinetic Strength Test}

In sports physiology and medical rehabilitation, isokinetic tests are performed for safe analysis of muscle strength ${ }^{17}$. The procedure used was to have the subjects stand with knees slightly flexed and pull on a dyna-band attached to the ground whose un-stretched length was no more than knee height. As they pulled and exerted force on the muscles mentioned in previous Sections, the eight EMG sensors sent signals back to the data 
acquisition and motion analysis systems. Details of EMG signals sent to the motion capture system are given in Section 3.6. With isokinetic testing, a baseline for maximum exertion of a particular muscle was established, which was then used for normalizing the muscle EMG data from the drop-jump tests.

\subsection{Acquisition of Static Reference Data}

Obtaining each subject's static global reference frame (Figure 5a) is the starting point of data acquisition. It is required to find the relative orientation of body segments to a common reference frame at any point in time. Local segment coordinate systems are embedded within the global reference frame. The combination of local segment

coordinate systems provides a baseline anatomical frame for the subject ${ }^{46,75}$. This provides a reference for the segment and joint coordinate systems themselves and its change in orientation from the anatomical reference frame is easily established during kinematic analysis. During the static trial, subjects were fitted with markers as shown in Figure $13[\mathrm{a}]$ and asked to stand with feet slightly apart at zero degrees of flexion and valgus. The duration of the static trial is generally limited to five seconds. This established the anatomical reference frame for the subject.

\subsection{Acquisition of Drop-jump Data}

The jumping activity for each subject consisted of drop-jumps heights of $30 \mathrm{~cm}, 40 \mathrm{~cm}$ and $50 \mathrm{~cm}$. First, each trial subject was fitted with reflective markers and EMG sensors as described in previous sections and in figure $13[\mathrm{~b}]$. The difference in marker placement between static and drop-jump trials is that markers located at the medial knee and ankle 
were removed. These markers are necessary at the static trial for constructing the anatomical reference frames; however during intense motion, there is possibility of interference with the detection of other knee and ankle markers. The remaining marker locations were unchanged.

A total of eight EMG sensors were used to measure action potential of muscles directly connected to and spanning the knee as detailed earlier, and in Figure 14. The purpose of their placement is to obtain extensor to flexor muscle ratios, one of the important variables linked to ACL damage. These values are then compared to those obtained from musculoskeletal simulations. The particular muscle ratios of interest to this study were the hamstring quadriceps (HQ) and the gastrocnemius quadriceps (GQ) ratios.

Starting with an elevation of $30 \mathrm{~cm}$, subjects performed three consecutive drop-jumps landing with both feet. The platform elevation was then increased by $10 \mathrm{~cm}$ and the exercise repeated until the maximum elevation of $50 \mathrm{~cm}$ was reached. EMG data collected during the trials were normalized as percentage of peak EMG obtained during the isokinetic tests.

\subsection{EMG Data Collection}

A wireless EMG collection system, from Delsys Inc., Columbus, $\mathrm{OH}$, was used for collecting EMG data from a total of eight muscle sites (Figure 14). Figure 15 shows the complete setup for obtaining EMG data using the Delsys Bagnoli EMG acquisition system. The individual electrodes strapped to muscle surfaces (above skin) send signals 
to amplifier modules through very thin flexible cables connected to a monitoring unit strapped to the subject's body. The monitor, called 'Myomonitor', communicates wirelessly with the host PC via a Local Area Network (LAN) connection. The LAN signals are intercepted by a host module through specific ports in the above PC, amplified and finally sent to the running motion analysis software (also in the host PC) via a National Instruments Data Acquisition (DAQ) system. Signal delay time ranges between $30-120 \mathrm{~ms}$ with a mean at $50 \mathrm{~ms}$.

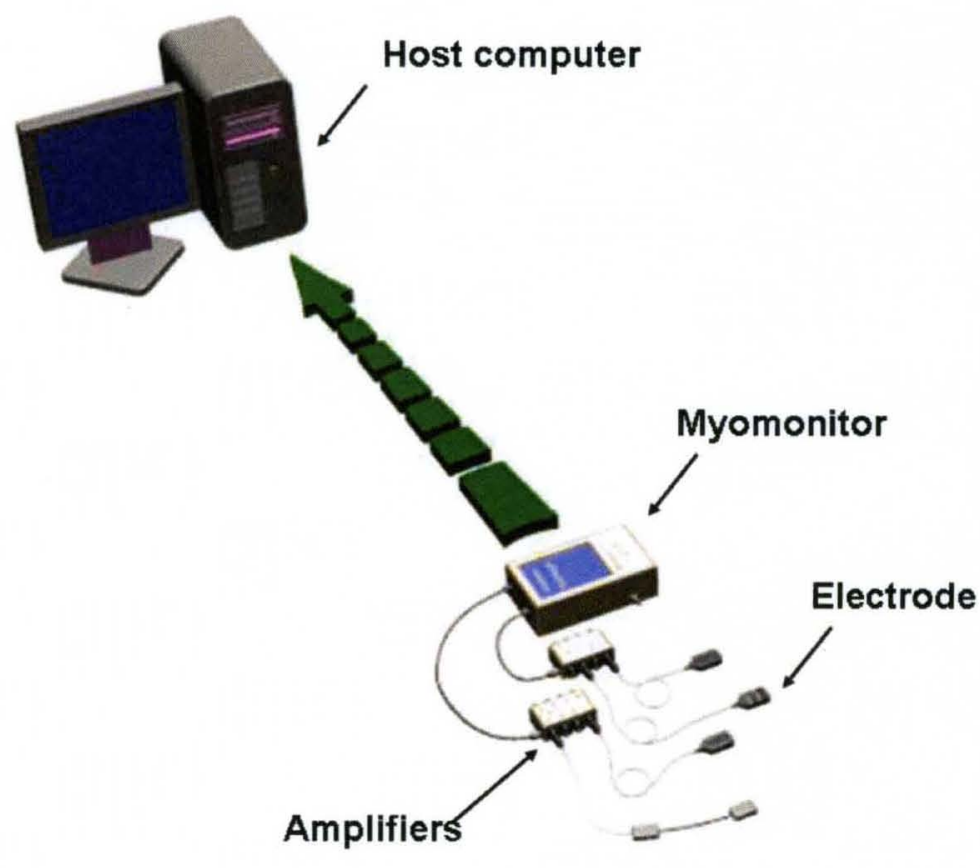

Figure 15. EMG arrangement with electrodes, amplifiers, hooked-up cables and hand (hip) held monitor with wireless onnection to the host.

Source. delsys.com/KnowledgeCenter/Practicum.html 


\subsection{A brief description of electrode function}

The electrode (Figure 16) housing is constructed from a waterproof polycarbonate plastic case, which is internally shielded to reject ambient electrical noise. The electrode contacts are made from $99.9 \%$ pure silver bars measuring $10 \mathrm{~mm}$ in length, $1 \mathrm{~mm}$ in diameter and spaced $10 \mathrm{~mm}$ apart.

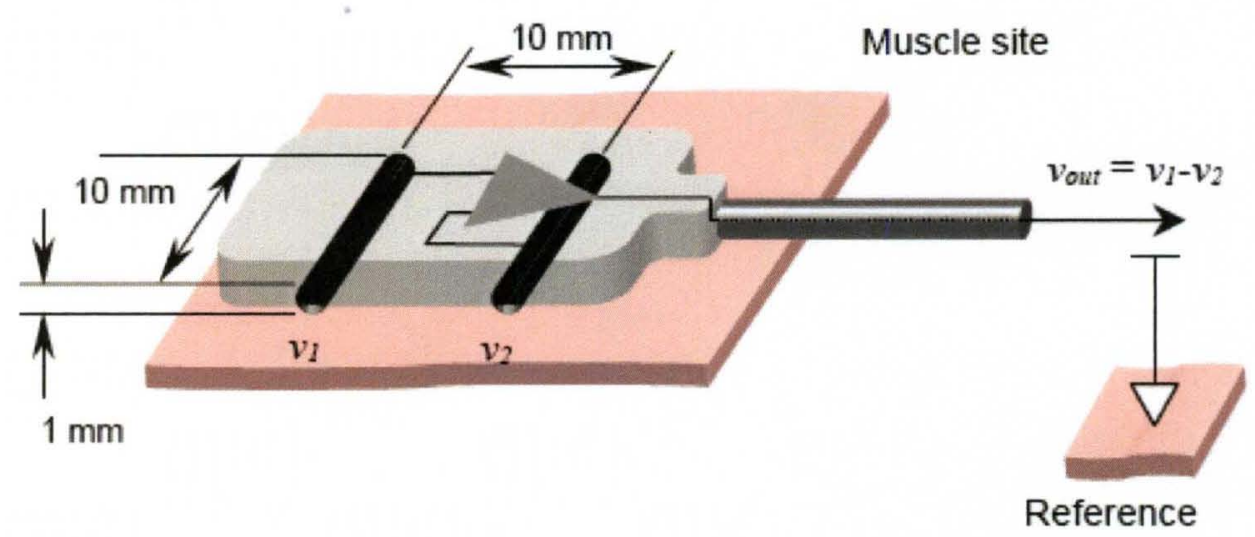

Figure 16. Delsys differential electrode placed on muscle tissue (pink). EMG signal is the result of the potential difference between the electrodes.

Source. delsys.com/KnowledgeCenter/Practicum.html

When placed directly in contact with the skin above an active muscle, these electrodes detect the EMG potential as the differential signal between them. The EMG potentials are always measured with respect to the electric potential of a neutral inactive site located away from the EMG muscle source. The electric potential of this neutral site is commonly termed "reference" potential, and is usually placed at a bony location of the subject, such as the sternum. 


\subsection{Post-Processing of Acquired Data}

After generating preliminary motion analysis data, kinematic and kinetic factors that influence knee joint motion were calculated. This required application of inverse dynamics to positional variables of the knee and their time derivatives. Inverse kinematic and kinetic processing were carried out with OpenSim. The list of variables related to possible ACL damage, detailed results of which are given in a later Chapter, is outlined in Table 1.

\begin{tabular}{|l|l|}
\hline \multicolumn{1}{|c|}{ Motion Property } & \multicolumn{1}{c|}{ Description } \\
\hline Angle $_{\text {knee flexion }}$ & $\begin{array}{l}\text { Time rate of change in knee flexion angle from T.D. to } \\
\text { recovery. }\end{array}$ \\
\hline Angle $_{\text {knee valgus }}$ & $\begin{array}{l}\text { Time rate of change in knee varus/valgus rotation angle } \\
\text { from T.D. to recovery. }\end{array}$ \\
\hline ACL Force $_{|c|} \mathrm{d}\left(\mathrm{ACL}_{\mathrm{strain}}\right)$ & ACL fiber forces between TD and recovery. \\
\hline ACL $_{\text {strain }} \mathrm{dt}$ & $\begin{array}{l}\text { ACL strain and time rate of change in ACL strain from } \\
\text { T.D. to recovery. }\end{array}$ \\
\hline Activation $_{\text {lexir }}$ & Flexor over extensor muscle activation ratio. \\
\hline Activation $_{\text {extensor }}$ & $\begin{array}{l}\text { Knee flexion and valgus (internal rotation) moment from } \\
\text { T.D. to recovery. }\end{array}$ \\
\hline Moment $_{\text {knee flexion }}$, Moment $_{\text {knee valgus }}$ & $\begin{array}{l}\text { Maximum power absorbed by knee between T.D. and } \\
\text { recovery. }\end{array}$ \\
\hline Power $_{\text {knee }}$
\end{tabular}

Table 1: Kinematic and kinetic factors of knee behavior closely associated with possible increase in ACL loading. The temporal changes in these factors were recorded while subjects perform the drop jump. Here T.D. refers to toe touch down. Recovery is when the ground reaction force is approximately equal to a person's weight. 


\subsection{Musculoskeletal Simulation}

Musculoskeletal models created for individual subjects were used for recreating the dropjump trials within the simulation environment. The first step of the simulation was to transfer dynamic marker data from the motion analysis domain (Section 4.6), through coordinate transformation, to the simulation domain of OpenSim. The description of the sequence of simulation steps, computation, optimization and others is given in section later in this Chapter. A summary of the steps within the simulation used for this study is shown in Figure 17.

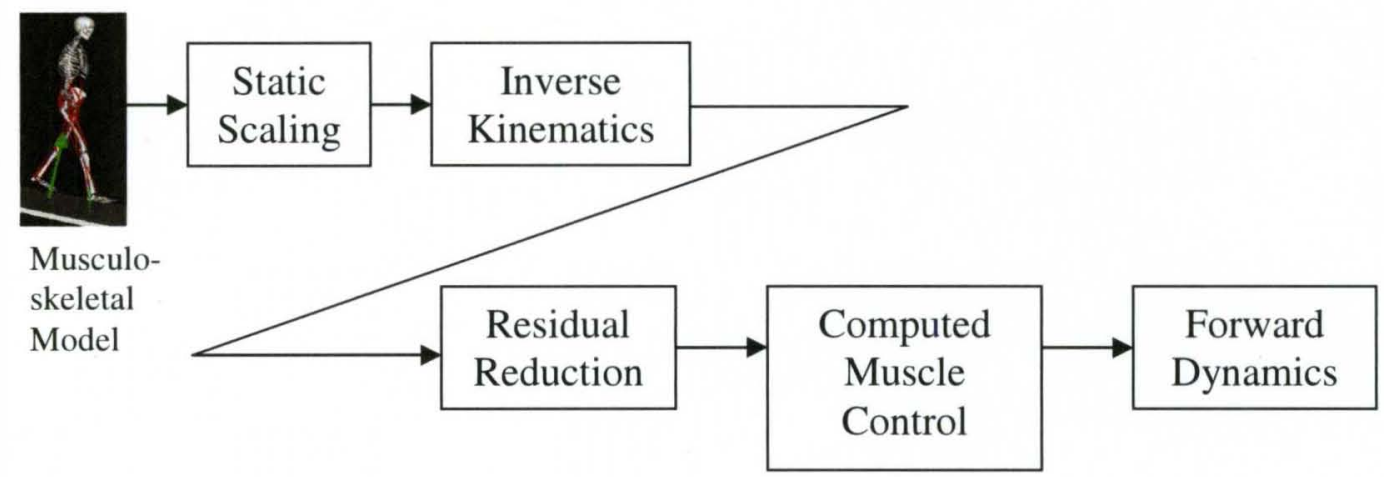

Figure 17. Flow chart showing sequence of tasks for dynamic simulation of the musculoskeletal model.

Figure 18 (over the page) shows the musculoskeletal model and the model's degrees of freedom that were used for this study. There were twenty-two rotational and three translational degrees of freedom. 

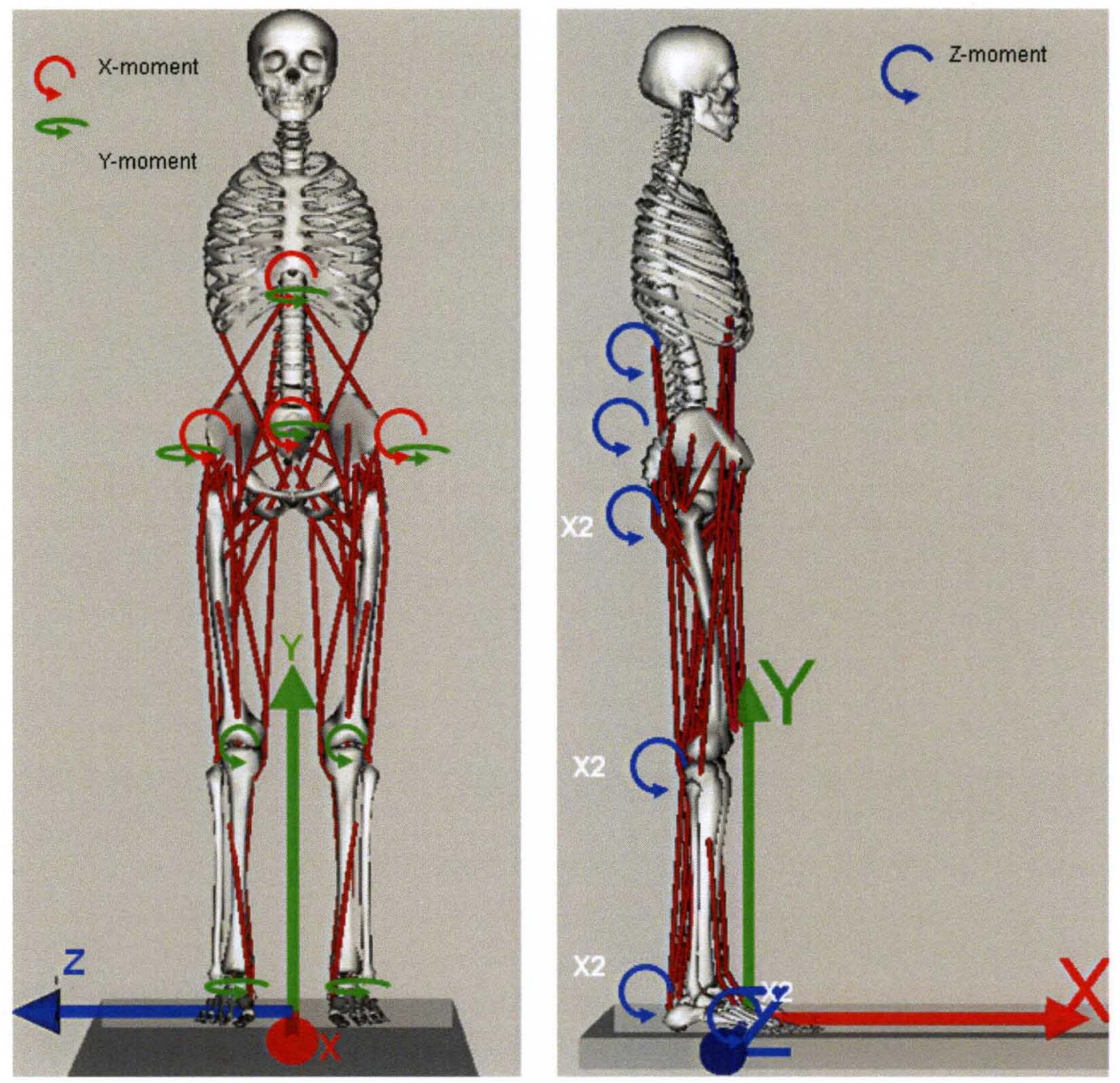

Figure 18. Musculoskeletal model showing the 22 rotational degrees of freedom and 3 translational degrees of freedom.

Figure 19 illustrates various position of the model after impact during the drop-jump trajectory for the between times toes-down (TD) to full recovery. Here full recovery implies the position where GRF returns to the subject's body weight. 


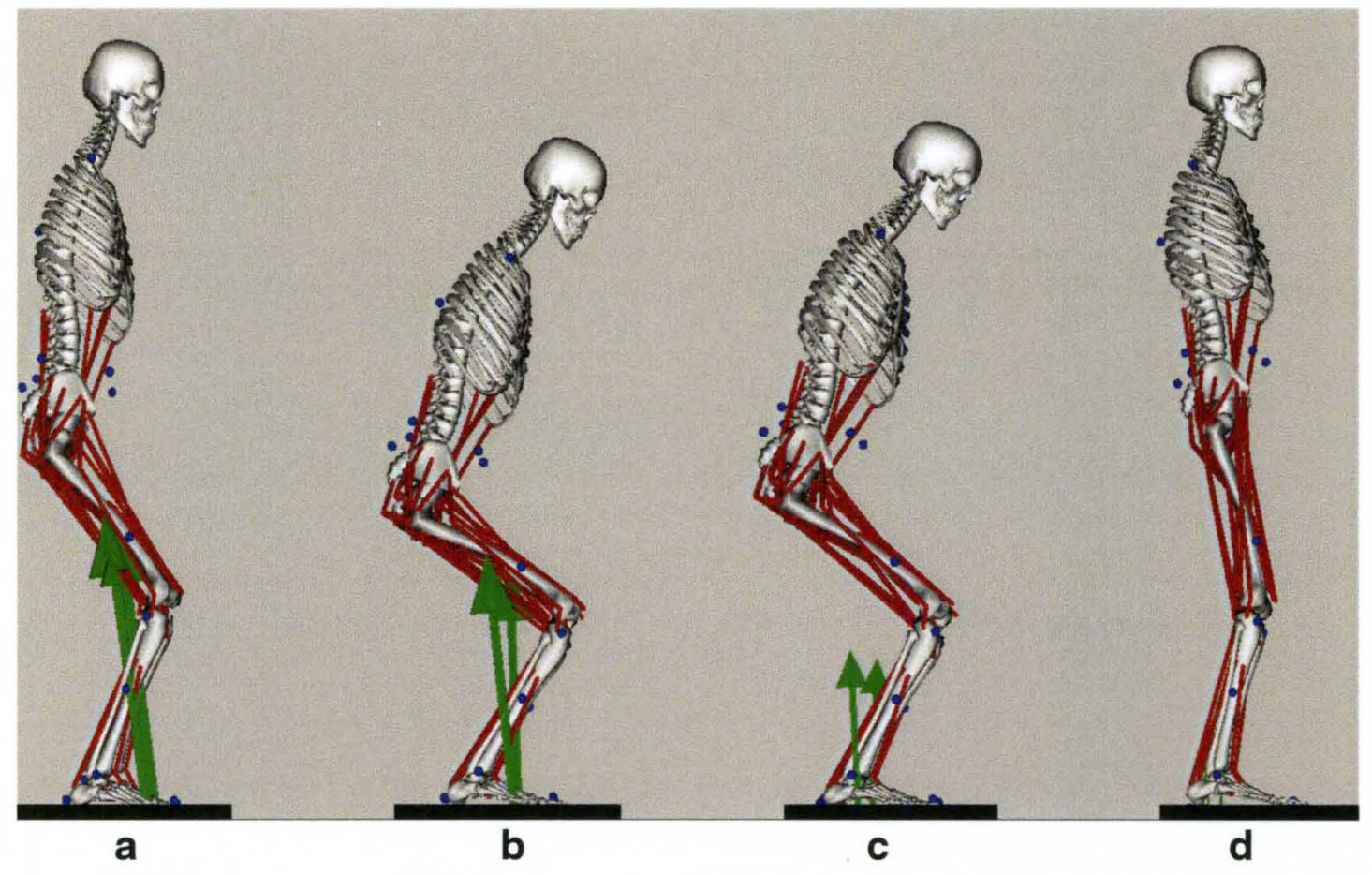

Figure 19. Force plate contact dynamics of a jump trajectory (30 $\mathrm{cm}$ jump height) using OpenSim 2.20 graphical interface showing ground reaction force (green) and various stances in chronological sequence, [a]: touch down, [b]: maximum crouch, [c]: recovering and [d]: recovered. Muscles are shown in red, body segments in grey, markers in blue and force plate in black.

\subsection{ACL Modeling}

The ACL insertion points are shown in Figure 20. The top part goes into the depths of the inter-condyloid part of the femur while the lower part is attached to the front meniscules of the tibia. 

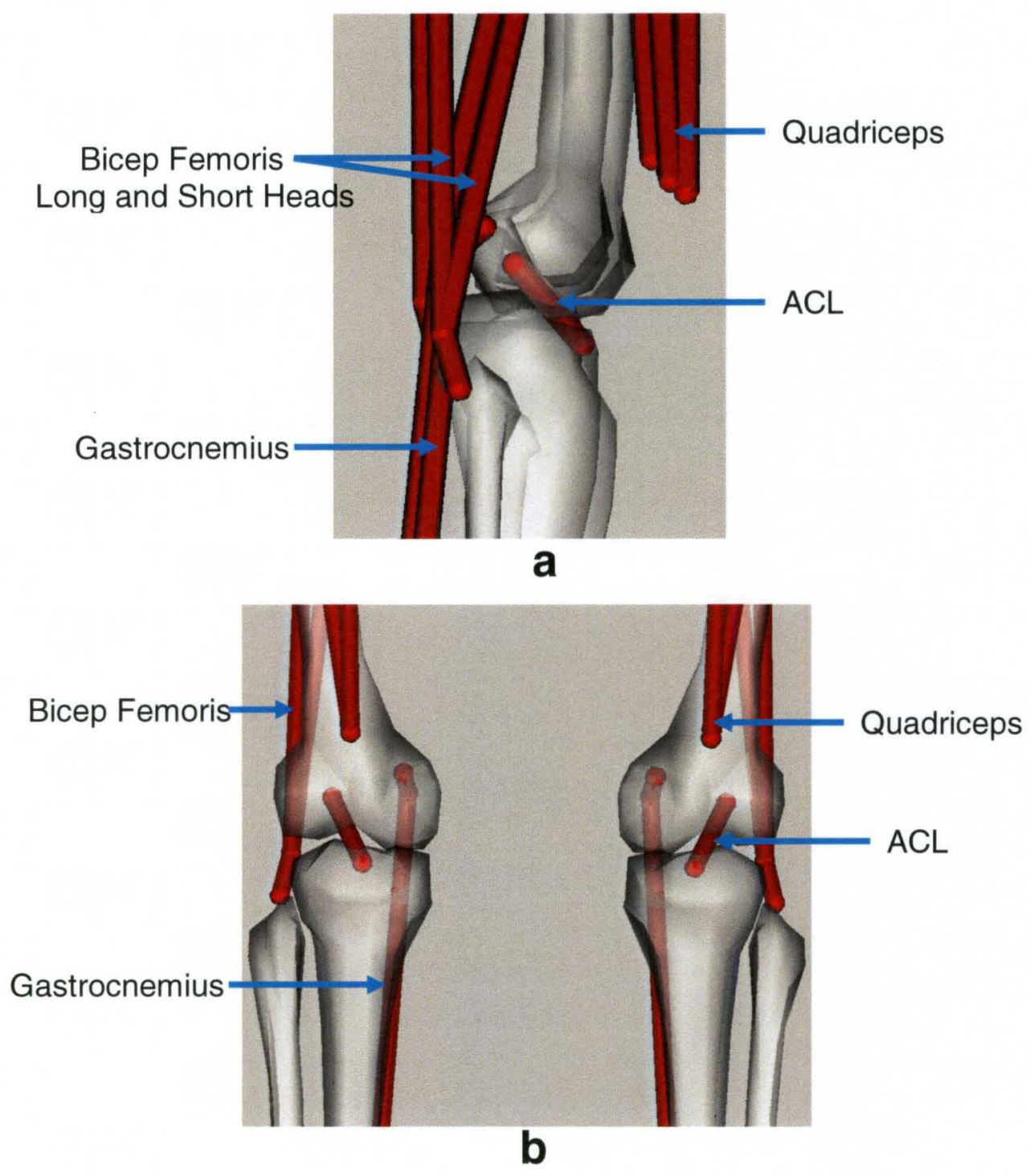

Figure 20. [a]: Frontal view of the ACL with insertion points into the inter-condyloidic eminence (femur) and the front meniscules (tibia). Muscles are shown in red and body segments in grey. [b]: Right sagittal view of the ACL.

\subsection{EMG Post-processing}

The simulated muscle activation data from OpenSim processing were pre-filtered with very high and low frequency noise etc. removed. However data collected from the Delsys system were raw. A filtering application written in $\mathrm{LabVIEW}^{\mathrm{TM}}, 2010$, from National 
Instruments, and using LabVIEW filtering sub-routines (called subVI) was used for processing the raw EMG data. The data were passed through a band-pass filter, allowing only a specified range of frequencies to pass. The bandwidth was set between $30-200 \mathrm{~Hz}$. It mainly removed low end electrical noise associated with wire sway and biological artifacts and high end tissue noise at the electrode site. A notch filter then removed 59-61 $\mathrm{Hz}$ electrical noise usually generated by base and other equipment and nearby electrical fixtures and appliances. Anti-aliasing was not used in the EMG filtering program since EMG modulating frequency was much higher at $1000 \mathrm{~Hz}$ than the frequency of interest $(30-200 \mathrm{~Hz})$. Figure 21 show the front panel of the LabVIEW filter module. The effectiveness of the bandpass and notch filters were verified by using spectral analysis which confirmed that post-filtered data was without any $60-62 \mathrm{~Hz}$ frequencies and were in the $30-200 \mathrm{~Hz}$ limit.

EMG data was then rectified and normalized with Maximum voluntary contraction (MVC) values from the same subject. The method to collect MVC was discussed earlier in section 3.3. Each subject performed three MVC exercises. RMS calculations recommended by DeLuca et al. in 2002, were then calculated on the peak MVC values and the highest of the three trials was used for normalizing ${ }^{22}$. Normalized EMG was obtained for left and right sides of each subject at each jump height and for the muscles shown in Figure 14. Finally Pearson correlation was used to establish the dependency between pairs of experimental and simulated activations. 


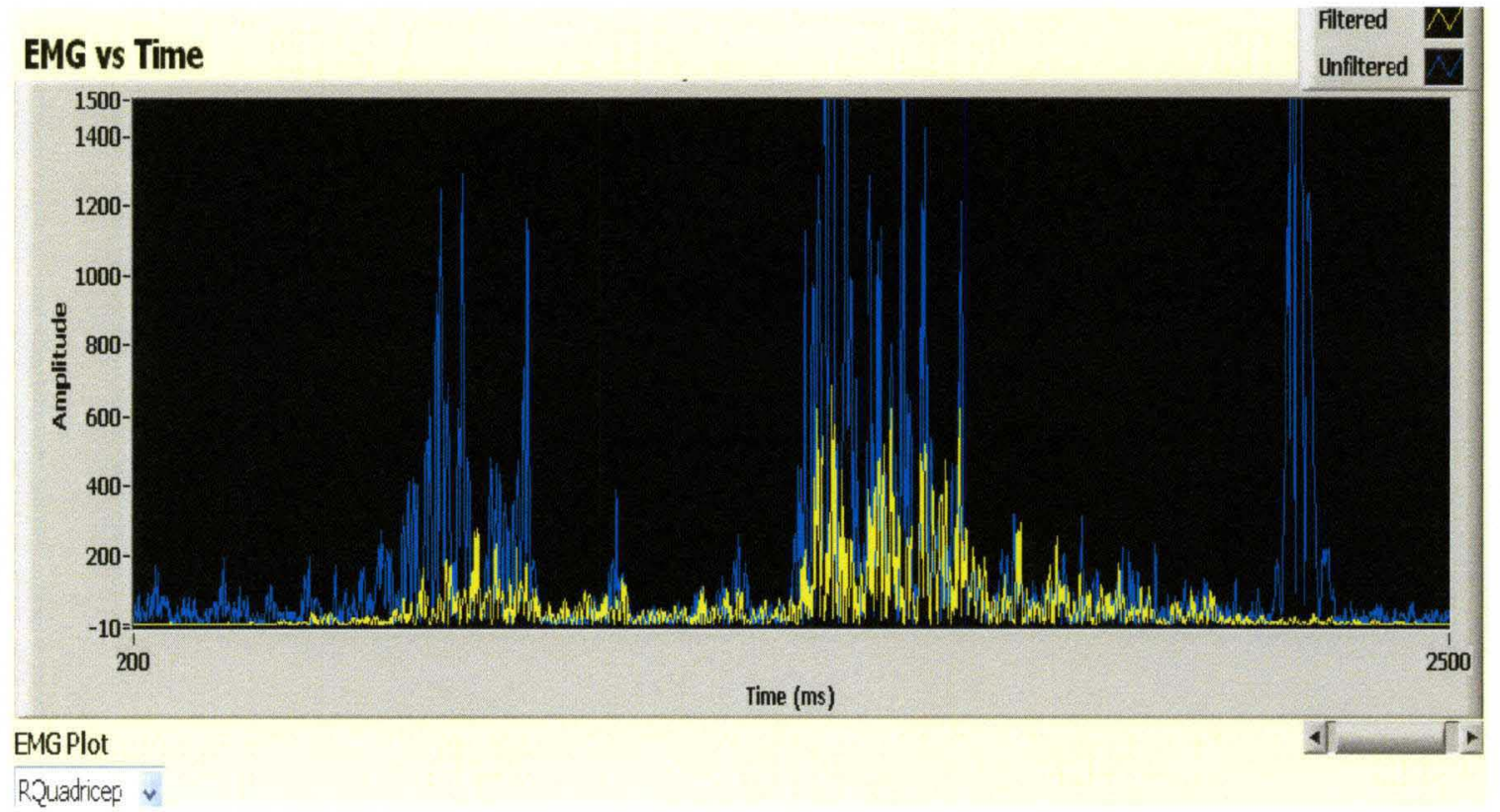

Figure 21. The front panel of National Instruments Labview ${ }^{\mathrm{TM}}$ VI for filtering data from experiments. The plot shows pre and post-filtered EMG for a single trial.

\subsection{Simulating increased knee valgus during landing}

The final step of this project was to study the effect of increasing knee valgus during landing, a convenient way to simulate the jumps with increased load on the ACL. The musculoskeletal models obtained for simulating the original jumps were used here as well. Valgus kinematics for the $50 \mathrm{~cm}$ jumps were changed by increasing the inward motion of the knee in the $\mathrm{x}$-direction as shown in Figure 22. The distance between the knees was decreased by fifteen percent from the original value. This amounted to approximately $20-25 \mathrm{~mm}$ of added knee displacement (inward) for both knees, for each (of the eleven) subject's $50 \mathrm{~cm}$ jump. 

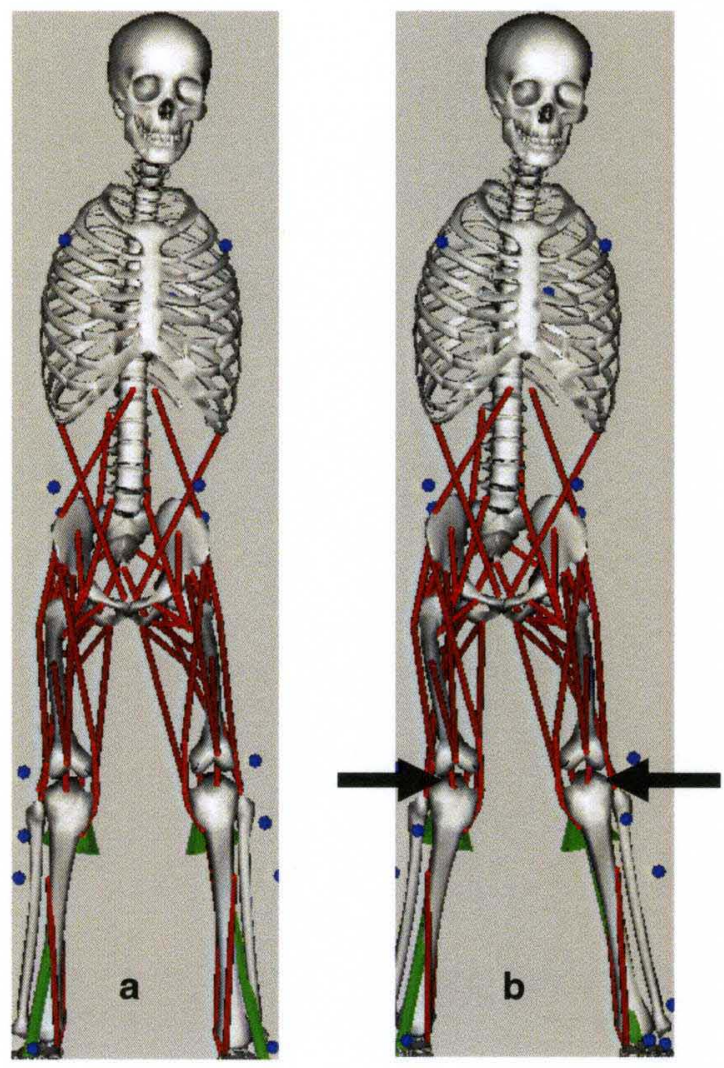

Figure 22. [a]. Original $50 \mathrm{~cm}$ stance and [b]. Valgus increase $50 \mathrm{~cm}$ stance by decreasing the horizontal distance between knees.

\subsection{Measurement of ACL Strain}

Studies published on ACL strains are generally varied in their techniques ${ }^{26,31,59,62}$. For example in a study published by Guoan et al. in 2005, using open-MRI, ACL strain is expressed as percentage of its length at full knee extension; that is when the tibiofemoral alignment is parallel (Figure 23) ${ }^{31}$. This study went on to show that at 90 degrees of flexion the ACL length decreased by $10 \%$ compared to its length at full extension. Butler et al. in 1986 showed ACL strain as percentage extension of shortest (slack) length. This in-vitro study showed that ACL rupture occurred around 13-14 \% strain of ACL slack length ${ }^{26}$. ACL strain calculations presented in the results are shown according to the later convention, where they are expressed as percentage extension of slack lengths. The 
reference or slack length for measuring strain was calculated when the model's knees were in a fully flexed (90 degrees) position.

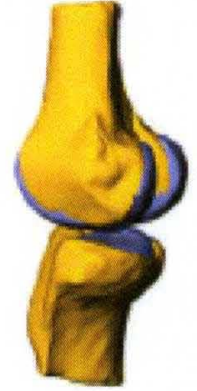

$0^{\circ}$

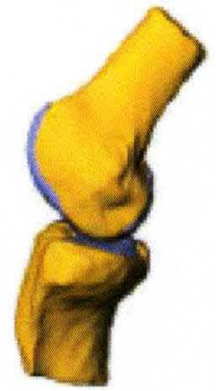

$30^{\circ}$

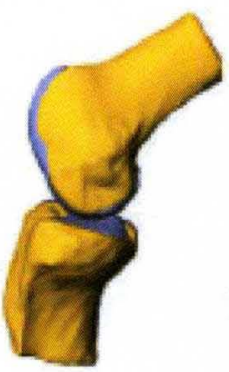

$60^{\circ}$

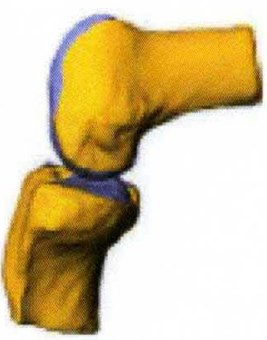

$90^{\circ}$

Figure 23. General positions of femur and tibia showing the direction in which knee is bent to determine changes in ACL length under tomography. ACL length is maximum at 0 degrees and minimum at 90 degrees.

Source. Guon et al., 2005.

\subsection{Validation Testing}

The Forward Dynamics generated kinematics were validated by correlating the experimentally obtained knee joint angles (flexion and valgus) for two of the musculoskeletal models to those knee angles obtained from Forward Dynamics simulation. The experimentally obtained knee angles (i.e. the knee joint angles from the subject's actual drop-jump) were calculated using the Inverse Kinematic tool of OpenSim. Pearson correlation, coefficient of determination and Pearson's P-values were calculated to validate the relationship between motion data obtained from actual dropjump performances and from Forward Dynamics simulation. 


\section{CHAPTER IV}

\section{RESULTS AND DISCUSSION}

With the steps and processes discussed in earlier Chapters, experiments were performed as detailed. Experimental data and simulation results are now presented, along with their analysis. First, examples of data from a single individual are shown, followed by kinematic and kinetic results from the simulations, generally averaged among all the eleven subjects. Most of the results presented are for variables at or around the knee, the ACL being the center of interest, along with variables that possibly affect it as listed in Table 1. Model validation test results are presented next. Finally, simulation results for added medial translation (valgus) for the $50 \mathrm{~cm}$ jump, for all eleven subjects, are presented and inferences are drawn.

It needs to be mentioned here at the outset that, in this Chapter, average peak values cited in text or tables are atemporal, averaged from the individual peaks of each of the eleven subjects, irrespective of their temporal position in the recovery period. Graphs (in Figures), on the other hand, are plotted with temporal averages among all eleven subjects, after positional scaling between TD and recovery (when the GRF returns to subject's body weight), and peaks in the graphs are just those, peaks of the temporal averages, rather than averages of the individual peaks. 


\subsection{Kinematic Analysis}

The Inverse Kinematics tool from OpenSim was used to obtain joint kinematics for the twenty-two rotational DOFs and three translational DOFs. Peak values for left and right knee flexion and abduction (valgus) angles (two DOFs for each knee) were obtained from all eleven subjects. An example of the temporal changes in knee flexion and valgus angles (of a single subject) during the trials is shown in Figures 24 and 25 respectively. The $\mathrm{x}$-axis values are percentage time based on the interval between TD and recovery, i.e., the point in time where GRF returns to body weight. These figures show that both valgus and flexion angles were much higher for the $50 \mathrm{~cm}$ jump.

An increasing trend with jump height was found for both, knee flexion and valgus angles, from peaks averaged among all eleven subjects, for each jump height. Average peak flexion angle for the $30 \mathrm{~cm}$ jump was $77.33 \pm 9.31^{\circ}$ for right knee and $76.63 \pm 8.90^{\circ}$ for left and it should be noted that all peak values in this Chapter are given with variance of one standard deviation. Average peak flexion angle for the $40 \mathrm{~cm}$ jump was $84.15 \pm 8.44^{\circ}$ for right knee and $84.16 \pm 8.18^{\circ}$ for left. Average peak flexion angle for the $50 \mathrm{~cm}$ jump was $83.80 \pm 7.35^{\circ}$ for right knee and $84.93 \pm 8.92^{\circ}$ for left. Average peak valgus angle for the $30 \mathrm{~cm}$ jump was $8.81 \pm 1.63^{\circ}$ for right knee and $9.29 \pm 1.46^{\circ}$ for left. Average peak valgus angle for the $40 \mathrm{~cm}$ jump was $9.67 \pm 1.07^{\circ}$ for right knee and $9.72 \pm 1.19^{\circ}$

for left. Average peak valgus angle for the $50 \mathrm{~cm}$ jump was $9.96 \pm 1.48^{\circ}$ for right knee and $10.21 \pm 1.04^{\circ}$ for left. Again, all these values were atemporal averages among eleven subjects. These values indicate that both average peak flexion and valgus angles generally increased with jump height. Close average peak values of the 40 and $50 \mathrm{~cm}$ 
flexion angles indicate that the knee flexion angle was reaching a threshold peak as the jump height increased.
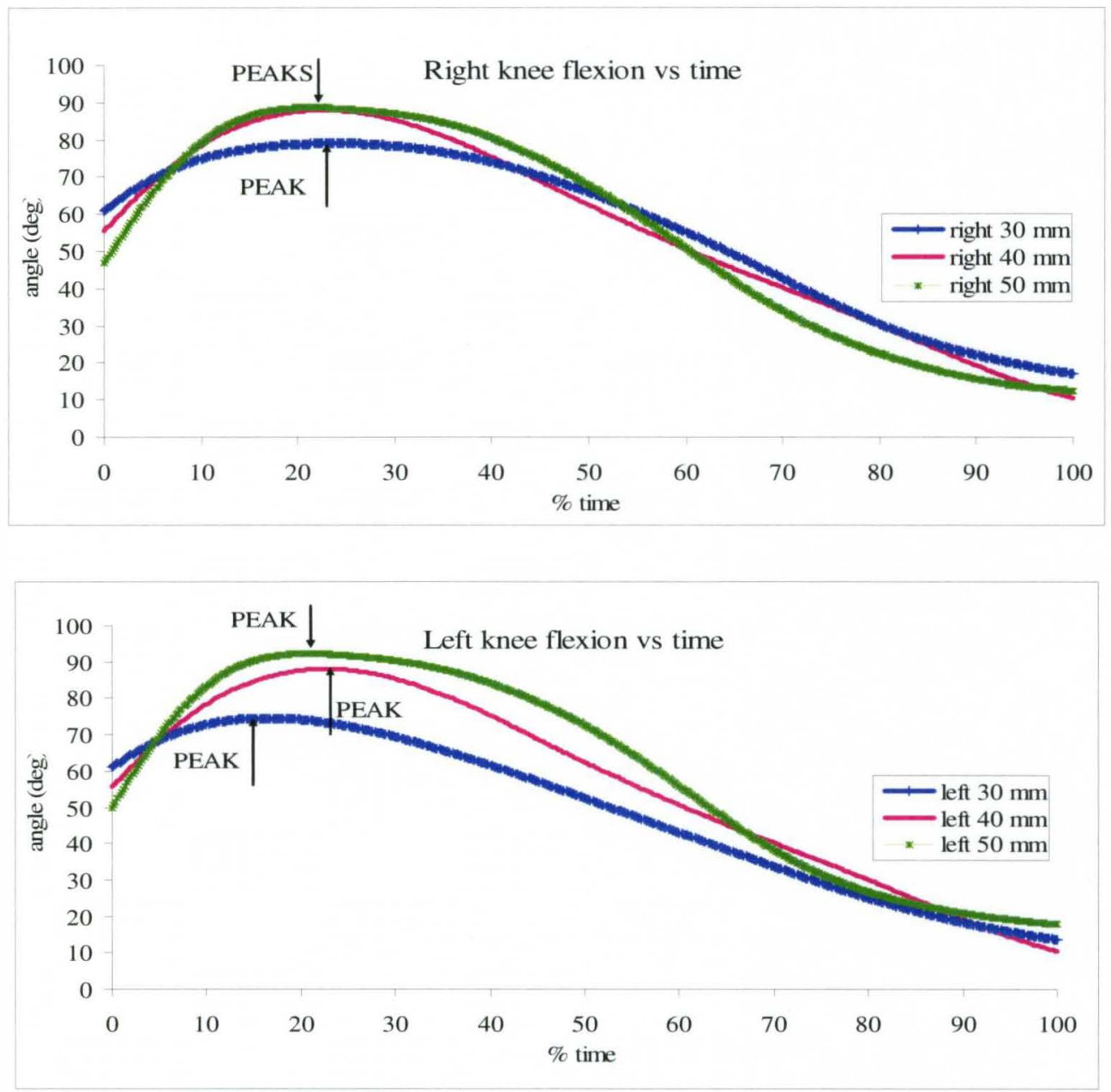

Figure 24. Right and left knee flexion angles against percentage time obtained for one subject for jump heights of $30 \mathrm{~cm}, 40 \mathrm{~cm}$ and $50 \mathrm{~cm}$. 

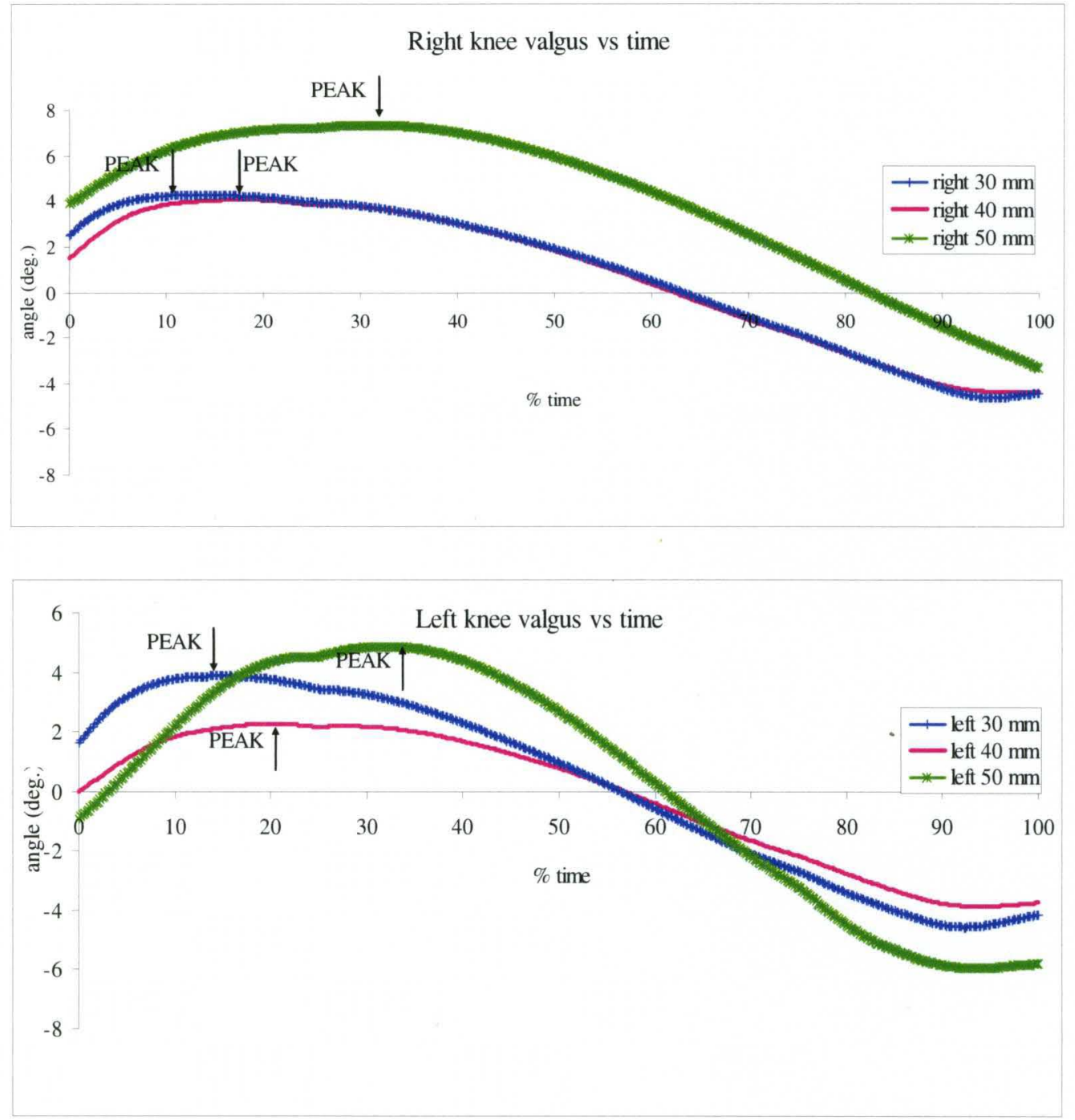

Figure 25. Right and left knee valgus angles against percentage time obtained for one subject for jump heights of $30 \mathrm{~cm}, 40 \mathrm{~cm}$ and $50 \mathrm{~cm}$.

\subsection{Ground Reaction Force}

Figure 26 shows temporal plots of the left and right vertical GRF $\left(F_{Y}\right)$ for a single subject. Temporal plots of GRF forces and ground reaction moments (GRM), averaged among all eleven subjects, for each of the three jump heights, are given in appendix E. 

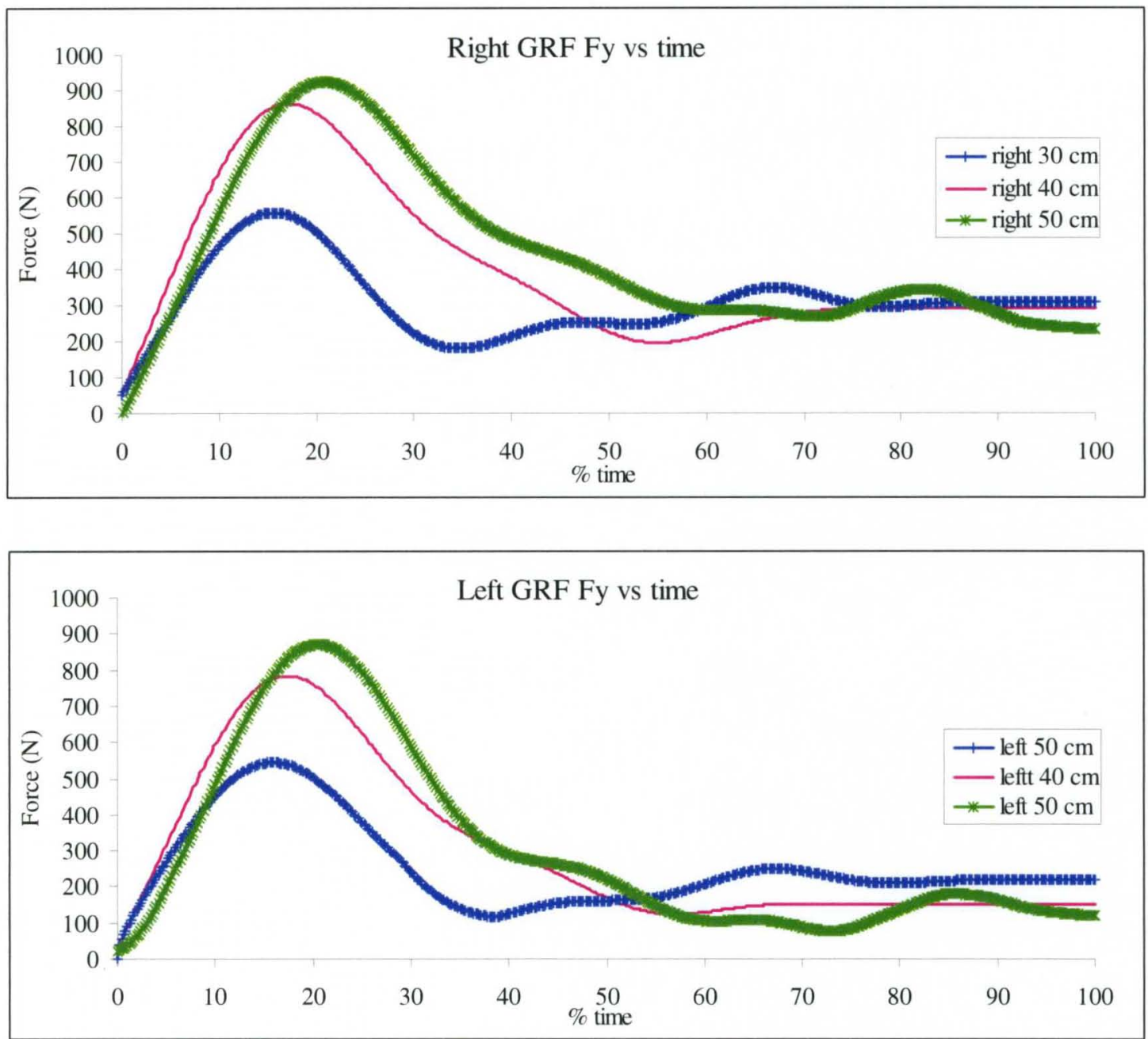

Figure 26. Right and left knee vertical (Fy) GRF forces against percentage time obtained for one subject for jump heights of $30 \mathrm{~cm}, 40 \mathrm{~cm}$ and $50 \mathrm{~cm}$.

Average peaks of GRF in the vertical ( $\mathrm{y}$-) direction and abduction (z-direction) GRM are given in Tables 2 and 3. The peaks were (atemporal) averages among all eleven subjects. It is seen that increases in average vertical GRF and abduction GRM were conservative. This observation is similar to the Burkhardt et al. 1990 study which showed that the increase in vertical GRF forces between 42 and $63 \mathrm{~cm}$ drop jump heights were small 
despite the nearly 1.5 times increase in jump height ${ }^{73}$. The figure from Burkhardt et al. is given in appendix $\mathrm{F}$.

\begin{tabular}{|c|c|c|}
\hline \multirow{2}{*}{$\begin{array}{c}\text { Jump Height } \\
(\mathrm{cm})\end{array}$} & \multicolumn{2}{|c|}{ Average Peak Vertical GRF (N) } \\
\cline { 2 - 3 } & Right & Left \\
\hline 30 & $657.30 \pm 94.12$ & $638.02 \pm 86.71$ \\
\hline 40 & $686.19 \pm 94.97$ & $661.24 \pm 94.53$ \\
\hline 50 & $712.70 \pm 93.77$ & $714.54 \pm 94.42$ \\
\hline
\end{tabular}

Table 2. Peak vertical ground reaction forces averaged among all eleven subjects.

\begin{tabular}{|c|c|c|}
\hline $\begin{array}{c}\text { Jump Height } \\
(\mathrm{cm})\end{array}$ & \multicolumn{2}{|c|}{$\begin{array}{c}\text { Average Peak GR Moment Mz } \\
(\mathrm{Nm})\end{array}$} \\
\hline & Right & Left \\
\hline 30 & $30.51 \pm 9.44$ & $-24.89 \pm 9.86$ \\
\hline 40 & $24.86 \pm 6.83$ & $-21.75 \pm 5.35$ \\
\hline 50 & $38.96 \pm 12.30$ & $-35.48 \pm 13.34$ \\
\hline
\end{tabular}

Table 3: Peak ground reaction abduction moments averaged among all eleven subjects.

\subsection{Musculoskeletal Simulation}

Musculoskeletal motion simulation was composed mainly of the static optimization, residual reduction and $\mathrm{CMC}$ steps. During the residual reduction step, body segment masses were adjusted for all eleven models so that segmental static residual forces could be minimized. Average adjusted body segment masses are presented in Table 4. 


\begin{tabular}{|c|c|}
\hline Body Segment & Average mass $(\mathrm{kg})$ \\
\hline Torso with head & $27.15 \pm 1.19$ \\
\hline Pelvis & $9.47 \pm 0.55$ \\
\hline Thigh (right) & $7.30 \pm 0.28$ \\
\hline Thigh (left) & $7.34 \pm 0.30$ \\
\hline Shank (right) & $2.98 \pm 0.20$ \\
\hline Shank (left) & $3.01 \pm 0.22$ \\
\hline Foot (right and left) & $1.20 \pm 0.04$ \\
\hline
\end{tabular}

Table 4. Average body segment masses from 11 subjects.

\subsection{EMG Data Processing}

Computing muscle activation (control) is the main funtion of CMC. This simulates flexor to extensor muscles ratios for HQ and GQ (Figure 14). To obtain the HQ ratios, normalized hamstring to quadriceps ratio for all subjects (from experiments and simulations) were calculated and temporal averages obtained. The same procedure was followed for obtaining GQ ratios. A general observation was that HQ ratios were higher and GQ ratios were lower in the simulation, compared to the experimental data, for all jump heights.

Pearson's correlations were obtained to determine the relation between average experimental and simulated $\mathrm{HQ}$ and GQ ratios as given in Table 5. Most of the correlations obtained were high, except for left HQ and GQ ratios for the $30 \mathrm{~cm}$ jump and the right $\mathrm{HQ}$ ratio for the $50 \mathrm{~cm}$ jump. Normally, positive correlations imply that strong associations exist between compared sets of values. In the Table, correlations marked with '*' have accompanying probability value $\mathrm{p}<0.05$, indicating that significant 
correlations exist between experiment and simulation for those jump height and ratio combinations.

\begin{tabular}{|c|c|c|c|}
\hline $\begin{array}{c}\text { Muscle } \\
\text { Ratio }\end{array}$ & $\begin{array}{c}\text { Jump height } \\
\text { (cm) }\end{array}$ & $\begin{array}{c}\text { Correlation } \\
\text { coefficient, } \mathbf{r}\end{array}$ & P-Value \\
\hline HQ (right) & 30 & $0.5268^{*}$ & $8.55 \mathrm{E}-03$ \\
\hline HQ (left) & 30 & -0.0584 & $2.30 \mathrm{E}-01$ \\
\hline GQ (right) & 30 & $0.4312^{\star}$ & $2.13 \mathrm{E}-02$ \\
\hline GQ (left) & 30 & -0.0996 & $2.09 \mathrm{E}-09$ \\
\hline HQ (right) & 40 & $0.4299^{*}$ & $2.00 \mathrm{E}-03$ \\
\hline HQ (left) & 40 & $0.2657^{*}$ & $2.71 \mathrm{E}-03$ \\
\hline GQ (right) & 40 & $0.3380^{*}$ & $2.89 \mathrm{E}-02$ \\
\hline GQ (left) & 40 & $0.3036^{*}$ & $3.62 \mathrm{E}-02$ \\
\hline HQ (right) & 50 & 0.0067 & $3.25 \mathrm{E}-02$ \\
\hline HQ (left) & 50 & $0.7026^{\star}$ & $4.67 \mathrm{E}-04$ \\
\hline GQ (right) & 50 & $0.3323^{*}$ & $1.79 \mathrm{E}-02$ \\
\hline GQ (left) & 50 & $0.8118^{*}$ & $1.76 \mathrm{E}-06$ \\
\hline
\end{tabular}

Table 5. Coefficients of correlation between experimental and simulated HQ and GQ ratios. * indicates significant positive correlations with $\mathrm{p}<0.05$.

\subsection{Forward Dynamics Simulation.}

Results of kinetic variables were obtained from the forward dynamic simulation of each drop-jump between T.D. and full recovery. ACL fiber lengths, strains and strain rates obtained from the forward simulation are shown in Figures 27-29. Reference ACL length for measuring strain was taken from each musculoskeletal model's ACL length at 90 degrees of knee flexion, as mentioned in section 3.13. The average slack length from the eleven subjects was $27.5 \mathrm{~cm}$. 

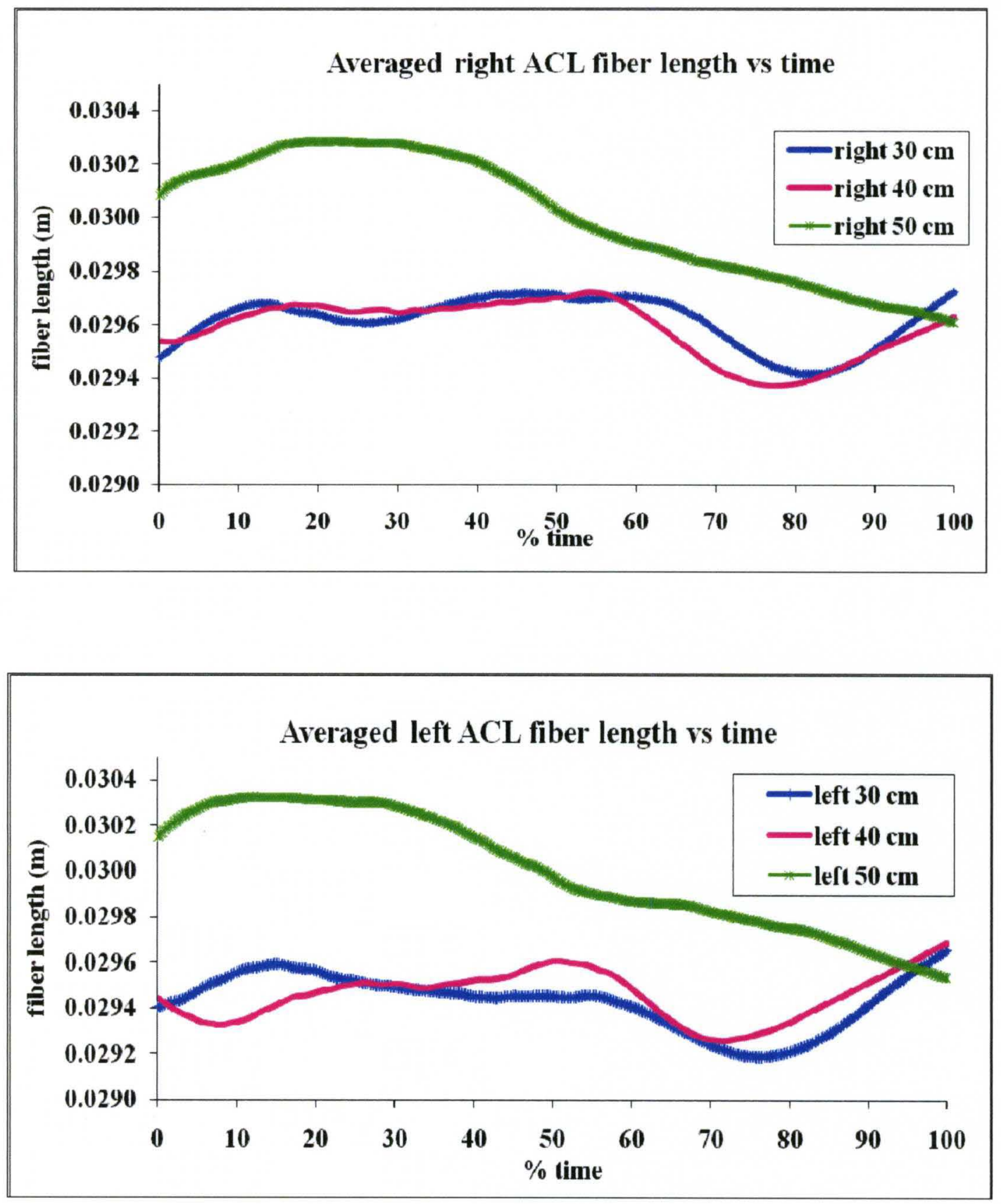

Figure 27. Average right and left ACL fiber lengths against percentage time obtained from eleven subjects participating in the drop-jump trials. The jump heights ranged from $30-50 \mathrm{~cm}$ in increments of $10 \mathrm{~cm}$. 

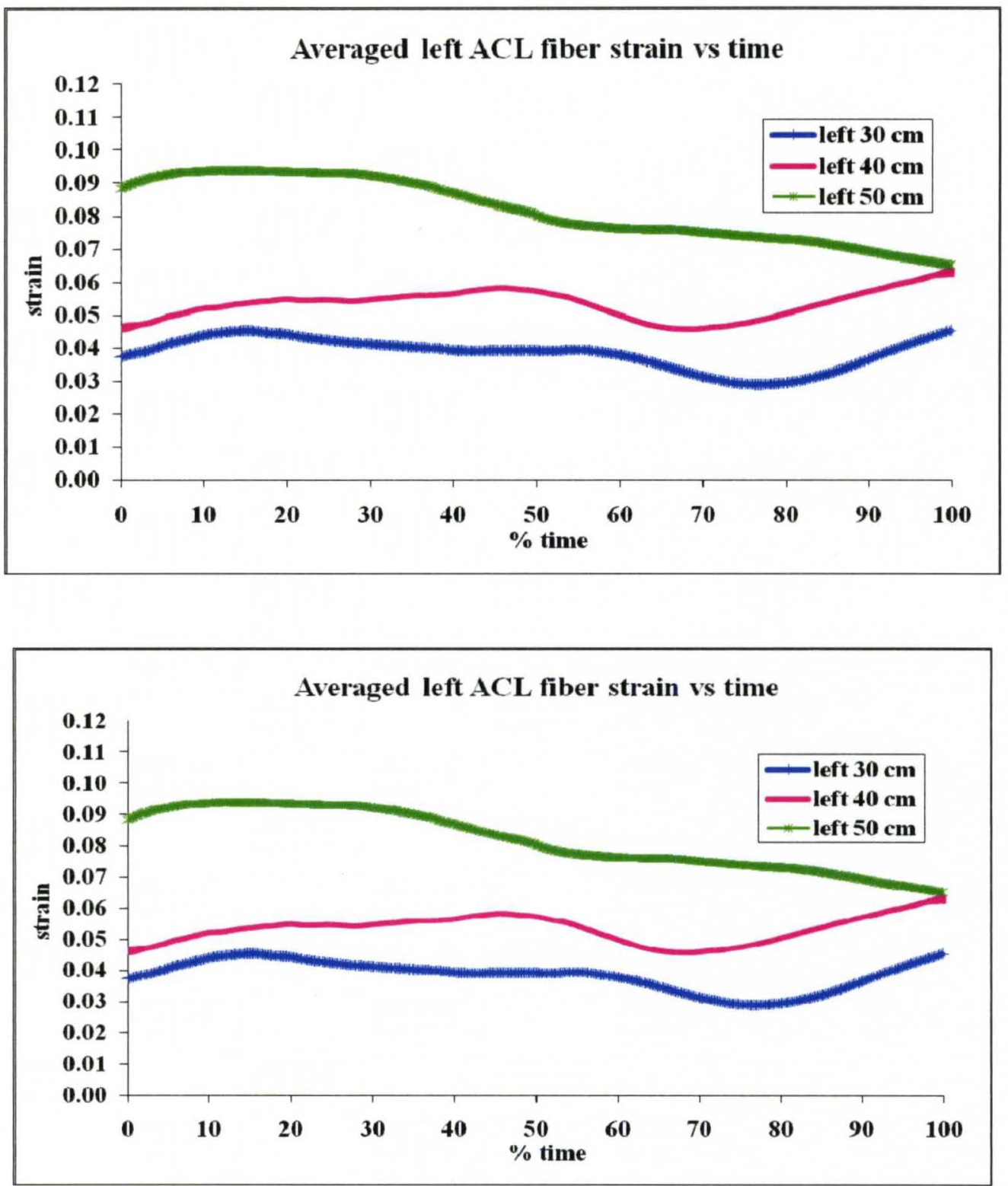

Figure 28. Average right and left ACL strains against percentage time obtained from eleven subjects participating in the drop-jump trials. The jump heights ranged from 30-50 $\mathrm{cm}$ in increments of $10 \mathrm{~cm}$. 

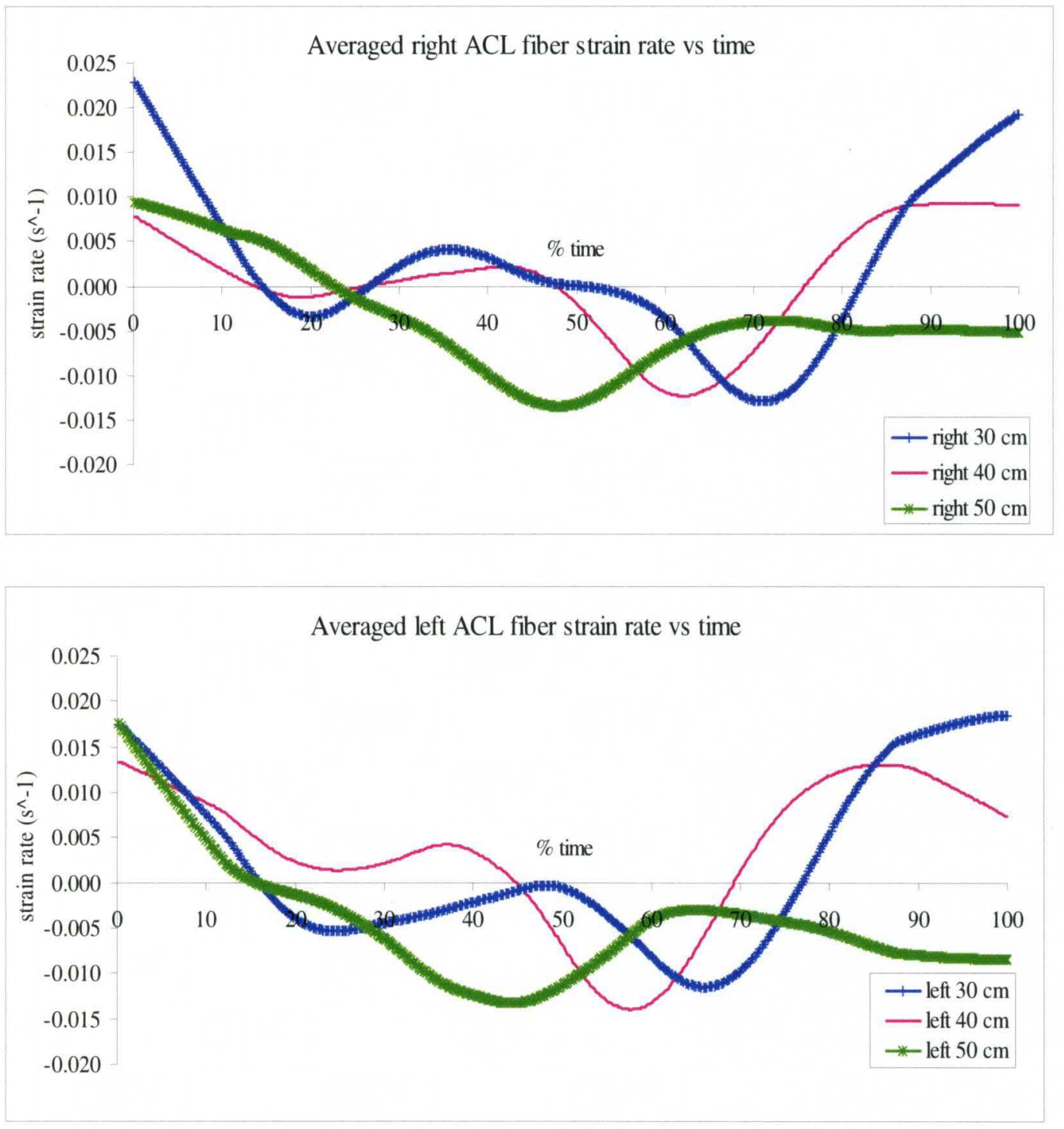

Figure 29. Average right and left ACL strain rates against percentage time obtained from eleven subjects participating in the drop-jump trials. The jump heights ranged from $30-50 \mathrm{~cm}$ in increments of $10 \mathrm{~cm}$.

From Figures 27 and 28, it can be seen that temporal average fiber length for the $50 \mathrm{~cm}$ trial was considerably higher than that at 30 and $40 \mathrm{~cm}$. Similarly there was increase in peak strain as jump height increased from 30 to $50 \mathrm{~cm}$, as shown in Table 6 . This indicates that while the ACL is expected to decrease in length when the knee is flexed 
with complete absence of valgus, it is not the case when valgus is present. Therefore risk of over-stretching the ACL exists if there is valgus. It should be noted that the peak fiber strains were averages of the peaks of eleven subjects, whereas graphs in Figure 28 show peaks of temporal average strains between TD and recovery for all subjects. The standard deviation provided in table 6 is indicative of the error limits for the entire trajectory (in Figure 28). Hence error indicators have not been provided for figures. This trend can be assumed for the remaining of this Chapter.

\begin{tabular}{|c|c|c|}
\hline $\begin{array}{c}\text { Jump } \\
\text { Height }(\mathrm{cm})\end{array}$ & \multicolumn{2}{|c|}{$\begin{array}{c}\text { Average Peak Percentage Fiber } \\
\text { Strain }\left(1 / 1_{0}\right)\end{array}$} \\
\hline & Right & Left \\
\hline 30 & $9.34 \pm 3.09$ & $8.03 \pm 2.80$ \\
\hline 40 & $9.21 \pm 3.24$ & $7.99 \pm 3.02$ \\
\hline 50 & $13.67 \pm 1.12$ & $13.38 \pm 2.04$ \\
\hline
\end{tabular}

Table 6. Average peak ACL fiber strain for 30,40 and $50 \mathrm{~cm}$ jump heights obtained from eleven subjects.

Figure 29 indicates that for 30 and $40 \mathrm{~cm}$ jump heights, the strain rates began as positive, became negative and went to positive again. The $50 \mathrm{~cm}$ jump strain rate ia different since it started as positive strain rate but ended as negative strain rate, and unlike the other two jump heights, did not go back to positive.

Temporal averaged ACL forces obtained from the forward dynamic simulation of the trials are given in Figure 30 . For the $50 \mathrm{~cm}$ jump, the forces that were expected to 
decrease due to contraction of the ACL (and negligible valgus), have actually gone up due to the presence of valgus.
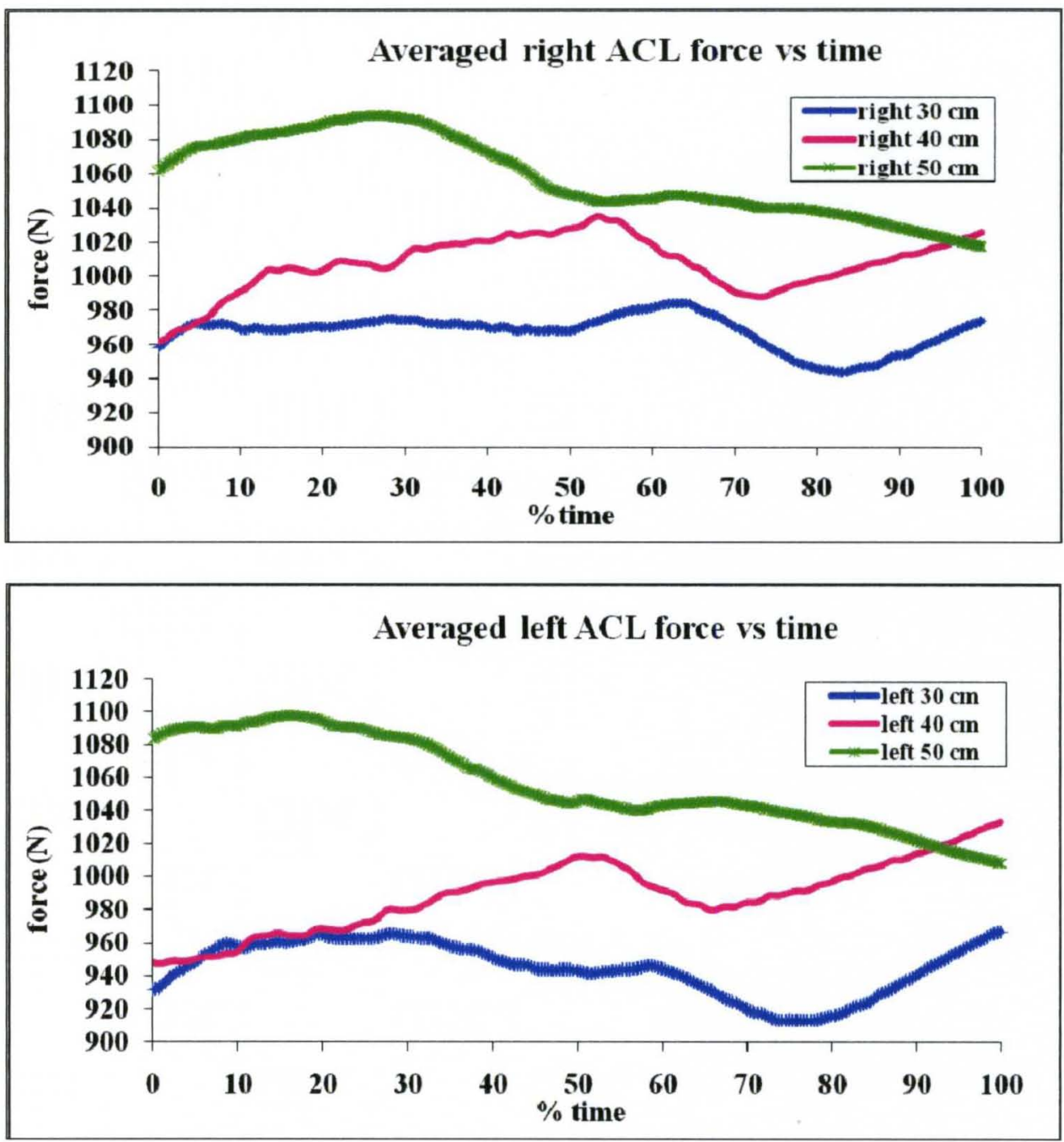

Figure 30. Average right and left ACL forces against percentage time obtained from eleven subjects participating in the drop-jump trials. The jump heights ranged from 30-50 $\mathrm{cm}$ in increments of $10 \mathrm{~cm}$.

Table 7 shows the atemporal average (among eleven subjects) peak fiber forces in the ACL for the three jump heights. An increase in peak average ACL forces is seen. Once 
again, it should be noted that peak fiber forces were averaged from the peaks of eleven subjects whereas in Figure 30 the peak is from the temporal average forces between TD and recovery for all subjects. Table 8 shows that strong correlations were found between temporal average ACL fiber force and displacement, which is typically expected from natural passive tissues.

\begin{tabular}{|c|c|c|}
\hline $\begin{array}{c}\text { Jump Ht } \\
(\mathrm{cm})\end{array}$ & \multicolumn{2}{|c|}{ Average Peak ACL Fiber Force (N) } \\
\cline { 2 - 3 } 30 & Right & Left \\
\hline 40 & $1056.09 \pm 71.39$ & $1043.09 \pm 61.33$ \\
\hline 50 & $1109.26 \pm 113.84$ & $1094.16 \pm 128.36$ \\
\hline
\end{tabular}

Table 7. Average peak fiber force for 30,40 and $50 \mathrm{~cm}$ jump obtained from eleven subjects.

\begin{tabular}{|c|c|c|}
\hline \multirow{2}{*}{$\begin{array}{c}\text { Jump Height } \\
(\mathrm{cm})\end{array}$} & \multicolumn{2}{|c|}{ Correlation Coefficient } \\
\cline { 2 - 3 } & Right & Left \\
\hline 30 & $0.86^{*}$ & $0.96^{*}$ \\
\hline 40 & $0.56^{*}$ & $0.59^{*}$ \\
\hline 50 & $0.98^{*}$ & $0.98^{*}$ \\
\hline
\end{tabular}

Table 8. Correlation coefficient between average fiber force and displacement for 30,40 and $50 \mathrm{~cm}$ jump heights. * indicates significant correlations with $\mathrm{p}<0.05$.

Presented in Figure 31 and 32 are the graphs of knee flexion and valgus moments, (temporal) average among the 11 subjects, for the 3 jump heights. 

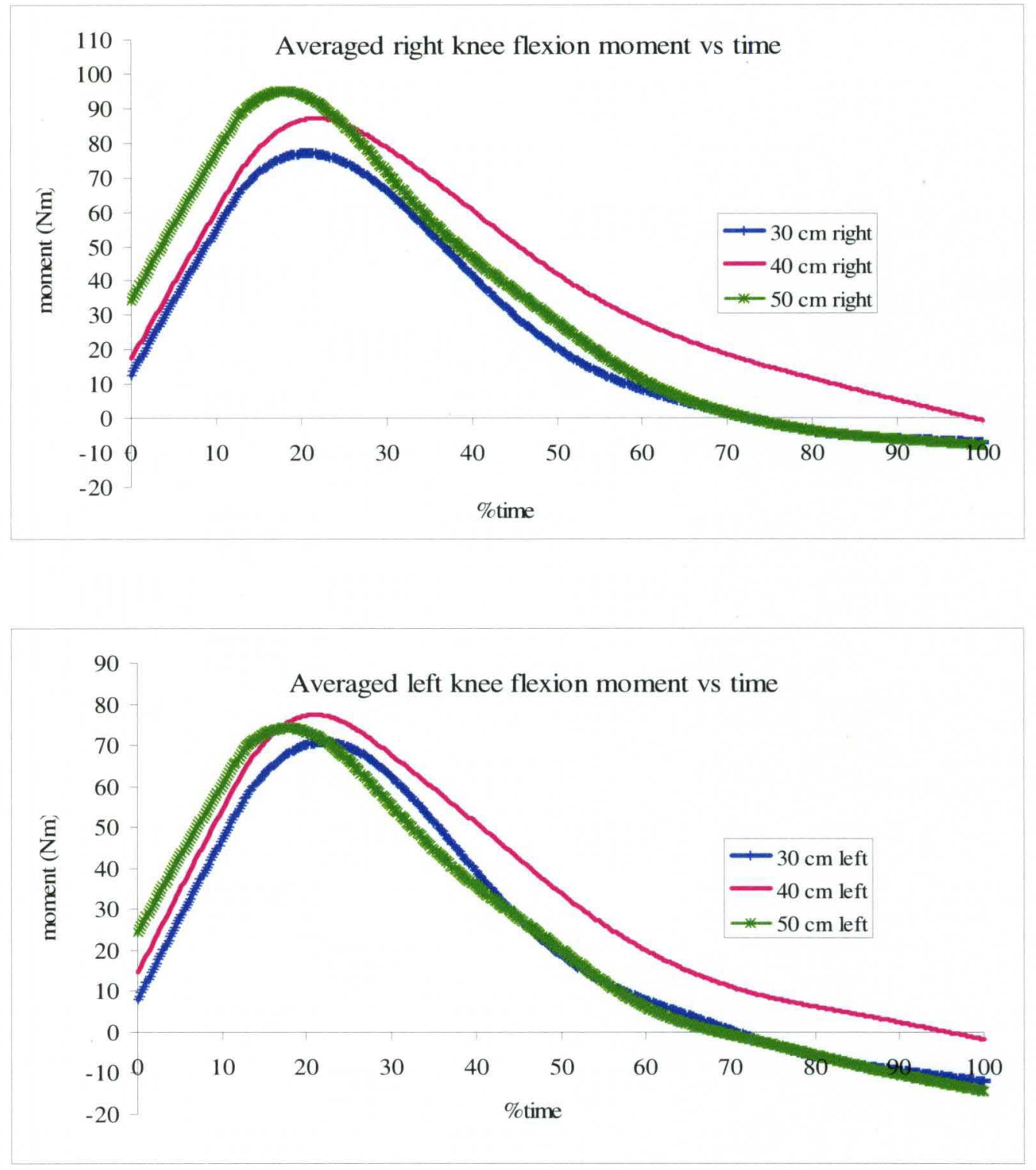

Figure 31. Average right and left knee flexion moments against percentage time obtained from eleven subjects participating in the drop-jump trials. The jump heights ranged from $30-50 \mathrm{~cm}$ in increments of $10 \mathrm{~cm}$. 

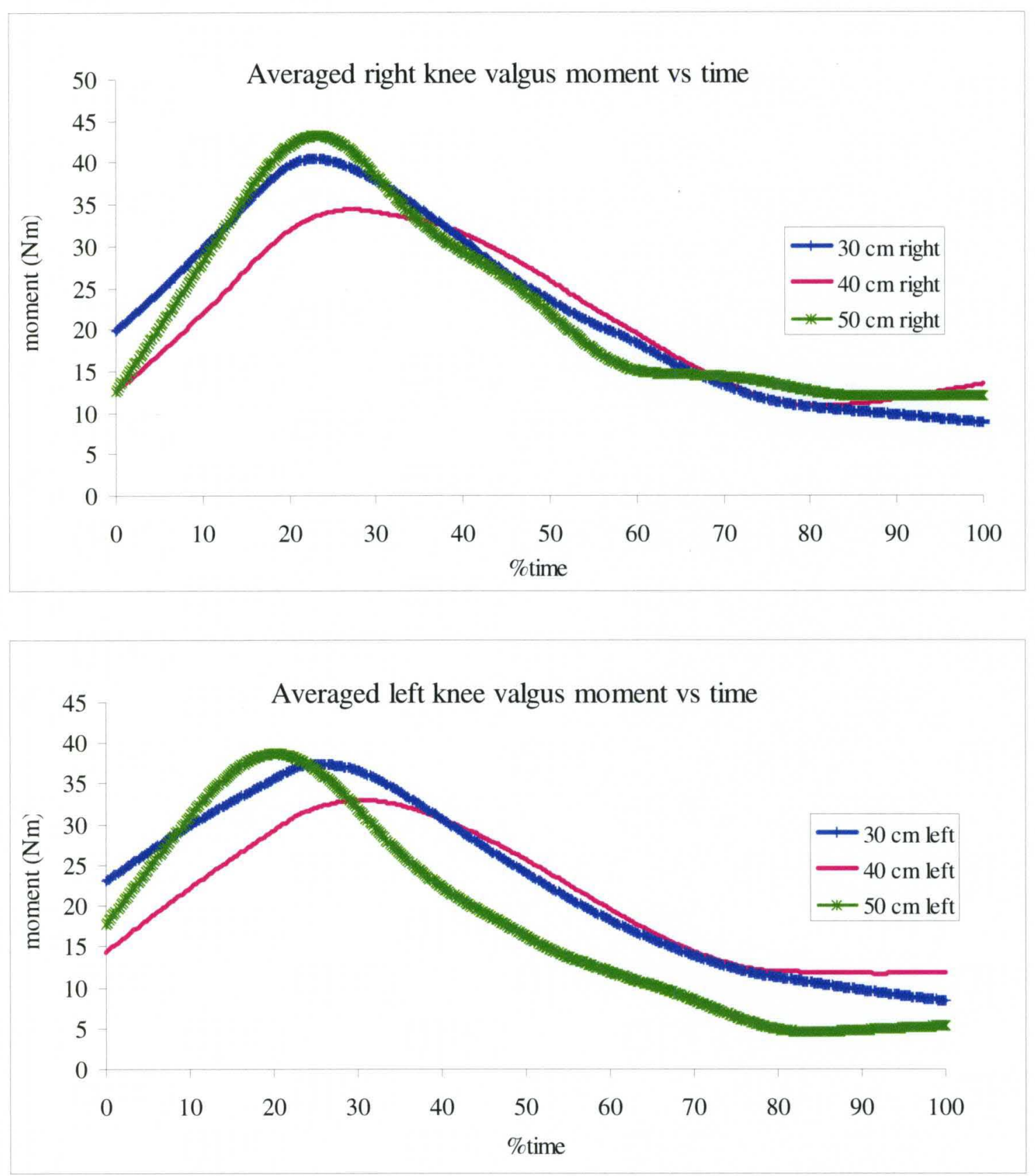

Figure 32. Average right and left knee valgus moments against percentage time obtained from eleven subjects participating in the drop-jump trials. The jump heights ranged from $30-50 \mathrm{~cm}$ in increments of $10 \mathrm{~cm}$.

Temporal average flexion and valgus knee moments obtained from the forward dynamic simulations are given in Figures 31 and 32. Increasing jump height did not produce large 
changes in valgus or flexion knee moments. A summary of average peak knee flexion and valgus moments for the three jump heights is given in Table 9. Like the average peak ACL forces and strains, the knee moments in Table 9 were atemporal averages from the peaks of eleven subjects. In contrast, the graphs in Figures 31 and 32 show peaks of temporal averages of moments between TD and recovery for all subjects.

\begin{tabular}{|c|c|c|c|}
\hline \multirow{2}{*}{$\begin{array}{c}\text { Jump } \\
\text { Ht }(\mathrm{cm})\end{array}$} & \multirow{2}{*}{ Direction } & \multicolumn{2}{|c|}{ Average Peak Knee Moment (Nm) } \\
\cline { 3 - 4 } & & Right & Left \\
\hline 30 & flexion & $91.04 \pm 16.68$ & $79.63 \pm 8.61$ \\
\hline 30 & valgus & $72.61 \pm 19.21$ & $67.81 \pm 25.15$ \\
\hline 40 & flexion & $106.09 \pm 15.17$ & $96.28 \pm 15.73$ \\
\hline 40 & valgus & $61.17 \pm 14.51$ & $45.85 \pm 9.89$ \\
\hline 50 & flexion & $110.00 \pm 17.96$ & $92.11 \pm 14.45$ \\
\hline 50 & valgus & $72.52 \pm 18.75$ & $75.15 \pm 28.3$ \\
\hline
\end{tabular}

Table 9. Average peak knee moments for 30,40 and $50 \mathrm{~cm}$ jump heights obtained from eleven subjects.

Table 10 shows the correlation coefficients between time average knee moments and knee angles for the three jump heights for both flexion and valgus. Significant correlations between knee moments and angles exist in each case. 


\begin{tabular}{|c|c|c|c|}
\hline \multirow{2}{*}{$\begin{array}{c}\text { Jump Height } \\
(\mathrm{cm})\end{array}$} & Direction & \multicolumn{2}{|c|}{ Correlation Coefficient } \\
\cline { 3 - 4 } & & Left & Right \\
\hline 30 & flexion & $0.91^{*}$ & $0.89^{*}$ \\
\hline 30 & valgus & $0.92^{*}$ & $0.93^{*}$ \\
\hline 40 & flexion & $0.94^{*}$ & $0.87^{*}$ \\
\hline 40 & valgus & $0.94^{*}$ & $0.92^{*}$ \\
\hline 50 & flexion & $0.93^{*}$ & $0.81^{*}$ \\
\hline 50 & valgus & $0.95^{*}$ & $0.92^{*}$ \\
\hline
\end{tabular}

Table 10. Correlation coefficients between knee moments and knee angles for 30, 40 and $50 \mathrm{~cm}$ jump heights. * indicates significant correlations where $\mathrm{p}<0.05$.

Figure 33 and 34 next show the temporal average power generated for the three jump heights. Both flexion and valgus powers were absorbed (negative) for the first $20 \%$ of total time but generated during the remaining $80 \%$ time. Both powers became zero towards the end of the recovery phase. This pattern was consistent in all jump heights.

Average peak knee flexion power (absorbed) for the $30 \mathrm{~cm}$ jump was $320.11 \pm 86.65 \mathrm{~W}$ and $266.22 \pm 49.26 \mathrm{~W}$ for right and left sides respectively. Average peak knee flexion power (absorbed) for the $40 \mathrm{~cm}$ jump was $411.93 \pm 57.45 \mathrm{~W}$ and $360.36 \pm 51.71 \mathrm{~W}$ for right and left sides respectively. Average peak knee flexion power (absorbed) for the 50 cm jump was $424.925 \pm 121.70 \mathrm{~W}$ for right side and $379.86 \pm 97.45 \mathrm{~W}$ for left. Average peak knee valgus power (generated) for the $30 \mathrm{~cm}$ jump was $44.36 \pm 14.14 \mathrm{~W}$ and 29.90 $\pm 13.66 \mathrm{~W}$ for right and left sides respectively. Average peak knee valgus power (generated) for the $40 \mathrm{~cm}$ jump was $49.39 \pm 5.70 \mathrm{~W}$ for right side and $29.15 \pm 12.05 \mathrm{~W}$ for left. Average peak knee valgus power (generated) for the $50 \mathrm{~cm}$ jump was $61.45 \pm$ 
$13.66 \mathrm{~W}$ for right side and $41.36 \pm 23.36 \mathrm{~W}$ for left. All these values, atemporal averages (among 11 subjects), indicate a small increase in peak power for both flexion and valgus.
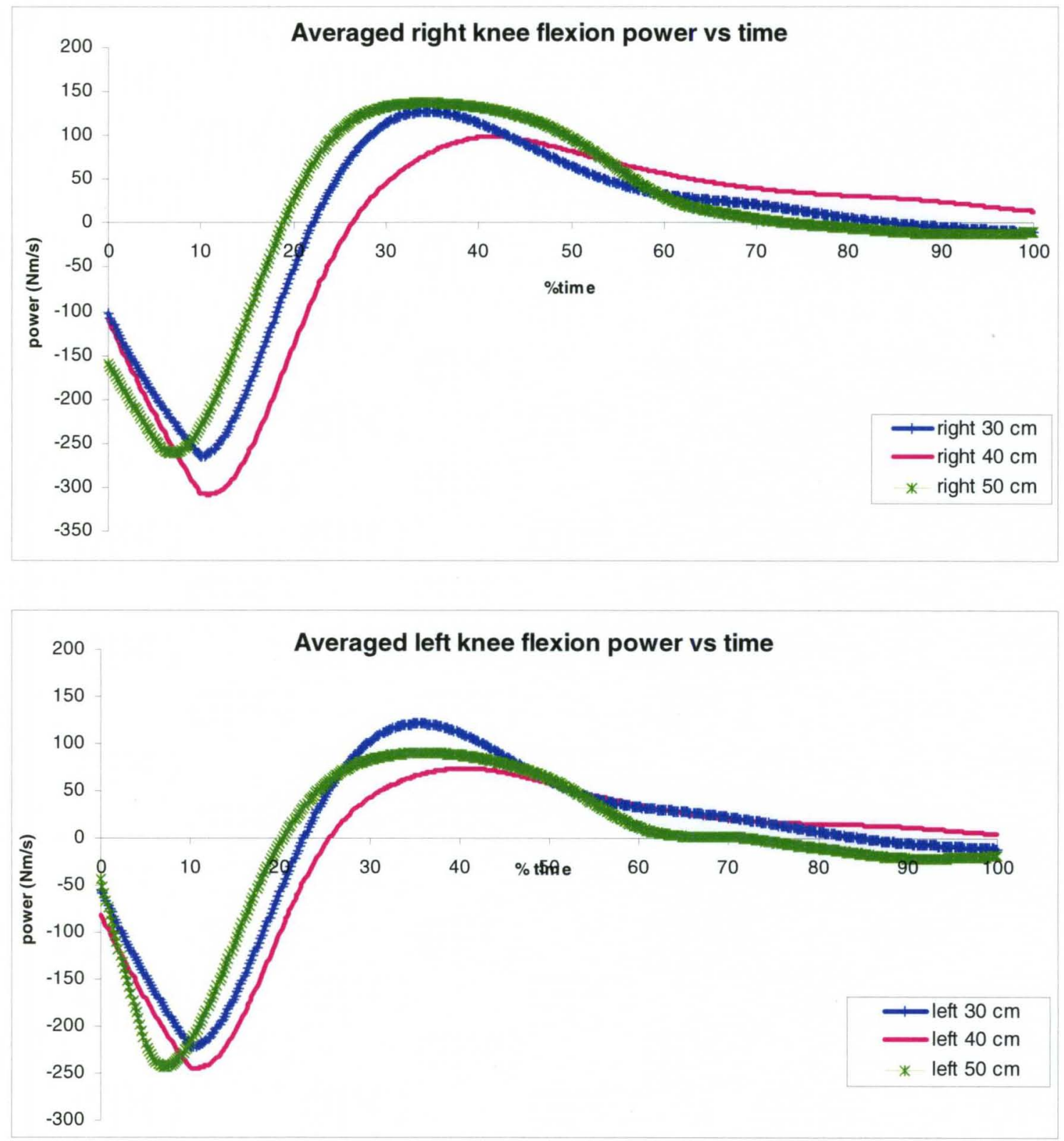

Figure 33. Average right and left knee flexion power against percentage time obtained from eleven subjects participating in the drop-jump trials. The jump heights ranged from $30-50 \mathrm{~cm}$ in increments of $10 \mathrm{~cm}$. 

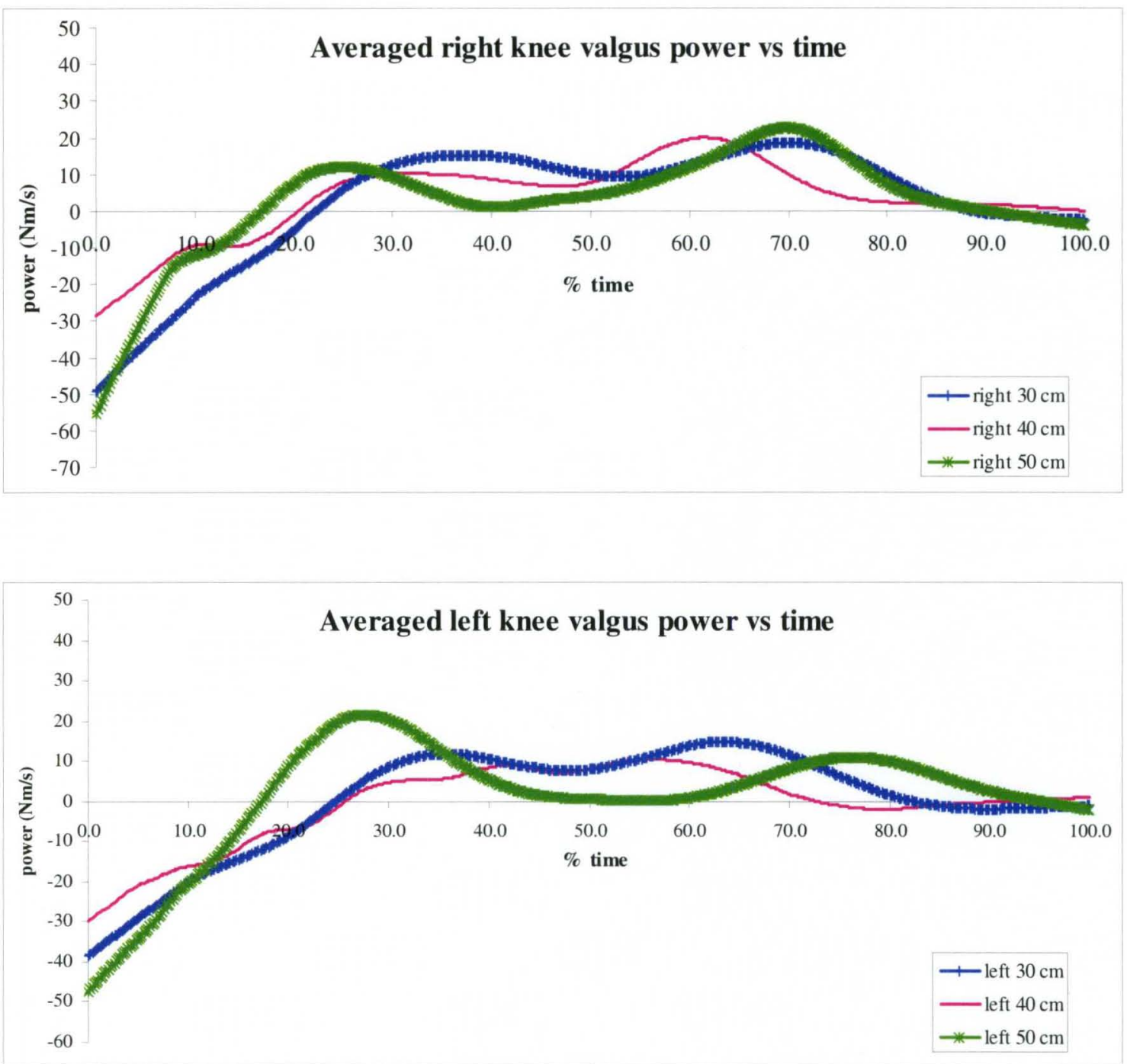

Figure 34. Average right and left knee valgus power against percentage time obtained from eleven subjects participating in the drop-jump trials. The jump heights ranged from $30-50 \mathrm{~cm}$ in increments of $10 \mathrm{~cm}$.

\subsection{Validation of Simulation Results}

The Forward Dynamics simulation results were validated by comparing knee joint angles (flexion and valgus) from the experimental data, and those computed with Inverse Kinematics, with the joint angles from Forward Dynamics analysis. Pearson's correlation coefficient, correlation of determination and Pearson's P-values were calculated for two 
subjects, for all jump height and both legs. Figures 35 and 36 show the flexion angles and Figure 37 shows the valgus angles at the $50 \mathrm{~cm}$ jump height, from both the subjects used for validation. It can be seen that subject $\mathrm{A}$ has higher and prolonged knee flexion and lower valgus than subject $\mathrm{B}$ and also that Forward Dynamics predicted knee kinematics from both subjects quite satisfactorily.
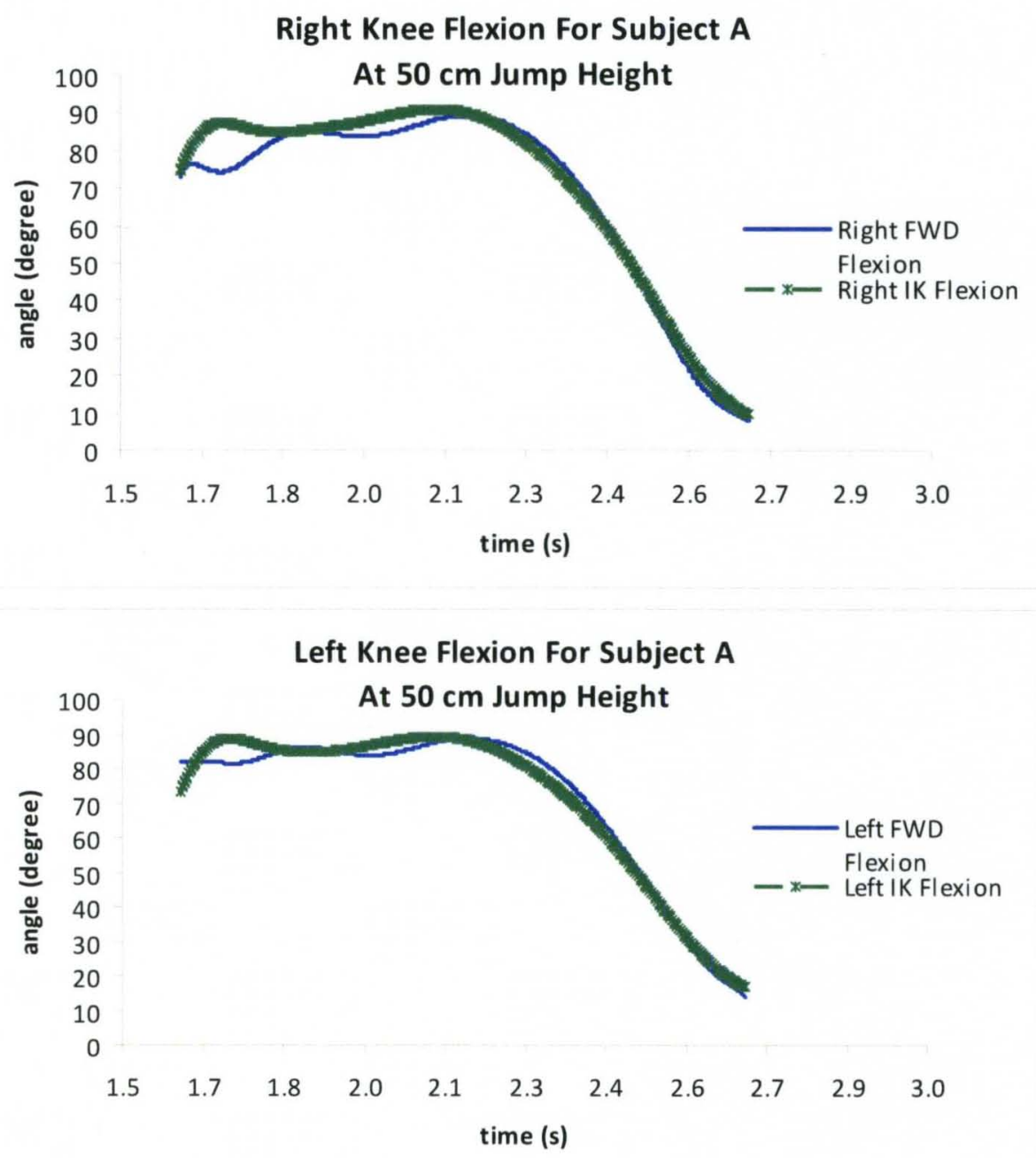

Figure 35. Right and left knee flexion vs time for subject A calculated with IK and Forward Dynamics analysis. 

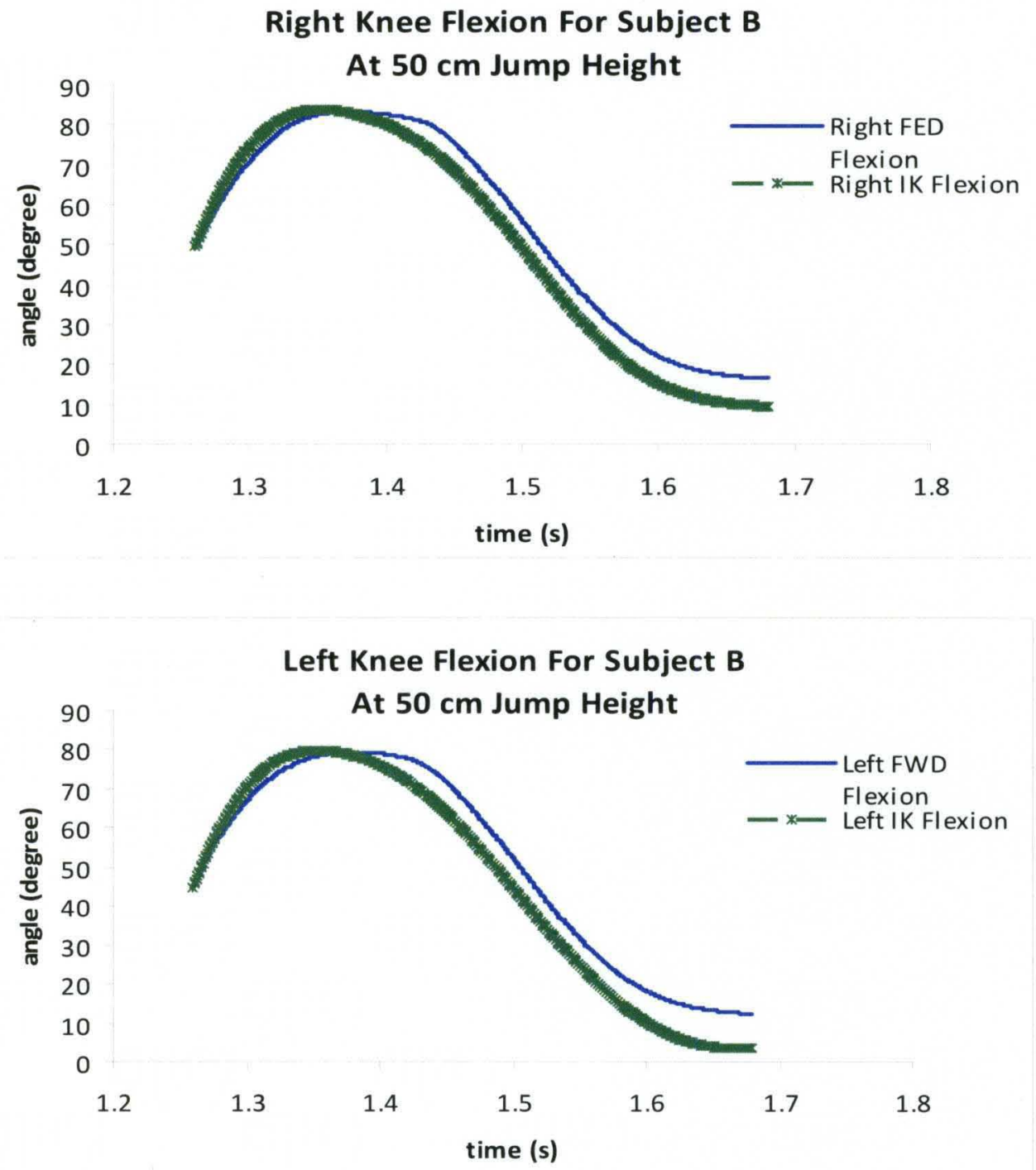

Figure 36. Right and left knee flexion vs time for subject B calculated with IK and Forward Dynamics analysis. 

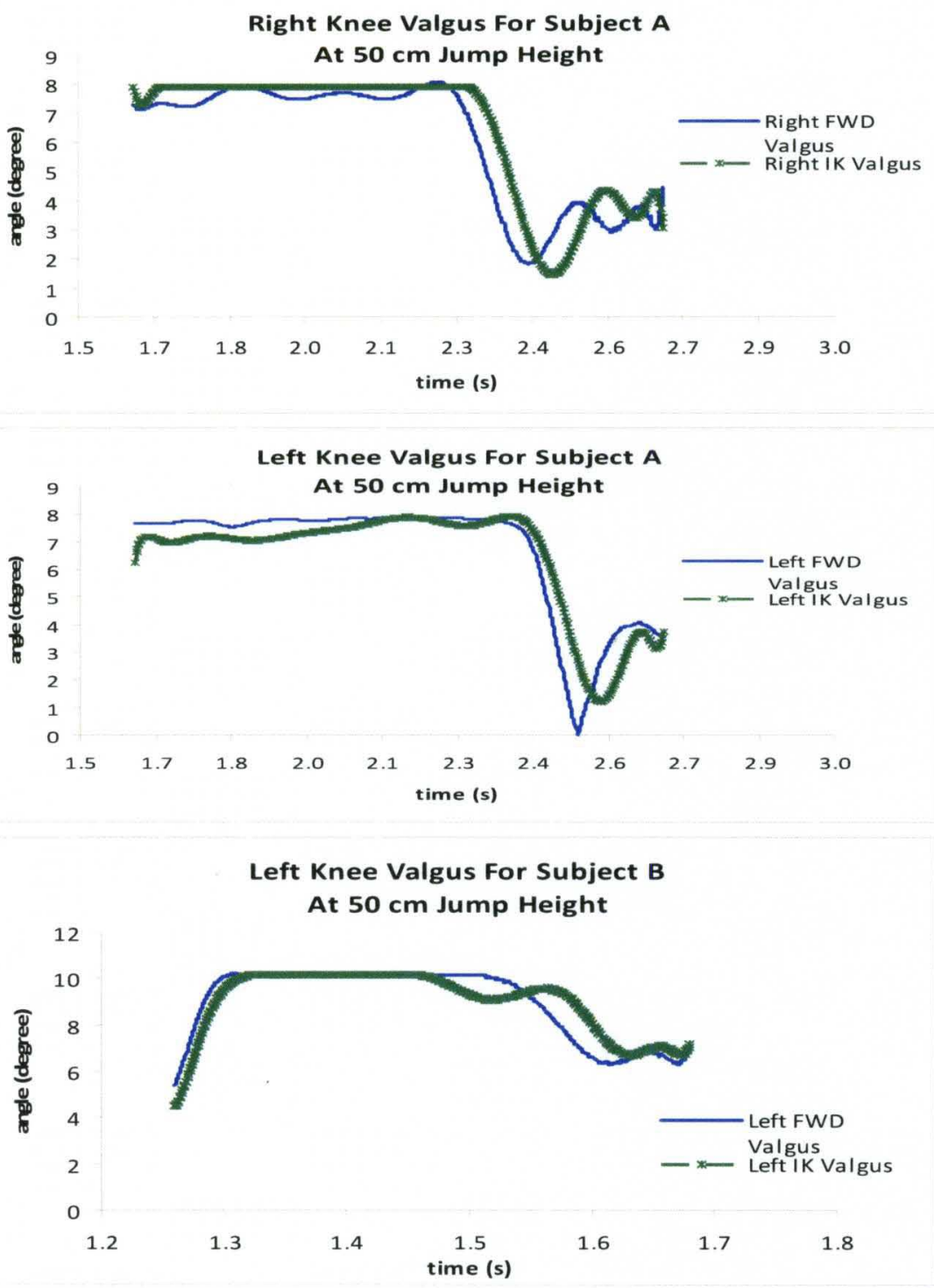

Right Knee Valgus For Subject B At $50 \mathrm{~cm}$ Jump Height

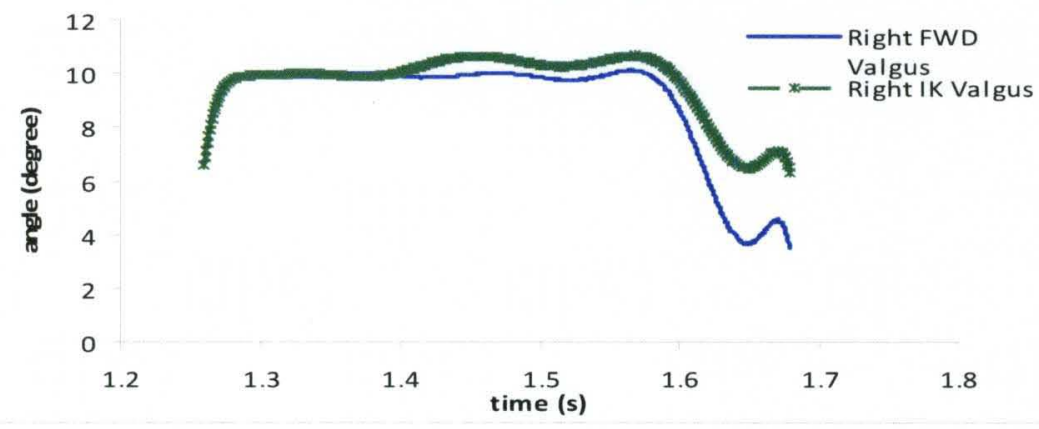

Figure 37. Right and left knee valgus vs time for subjects A and B calculated with IK and Forward Dynamics analysis. 
Table 11 displays the Correlation Coefficient and Coefficient of Determination from the joint angles comparison between Inverse Kinematics and Forward Dynamics.

\begin{tabular}{|c|c|c|c|c|c|}
\hline \multirow{2}{*}{$\begin{array}{c}\text { Jump } \\
\mathrm{Ht}(\mathrm{cm})\end{array}$} & Direction & \multicolumn{2}{|c|}{ Correlation Coeff. } & \multicolumn{2}{c|}{ Coeff. Of Determination } \\
\cline { 3 - 6 } & & $\mathrm{r}$ (right) & $\mathrm{r}(\mathrm{left})$ & $\mathrm{r}^{2}$ (right) & $\mathrm{r}^{2}$ (left) \\
\hline 30 & flexion & 0.9994 & 0.9830 & 0.9994 & 0.96626 \\
\hline 30 & valgus & 0.9722 & 0.9432 & 0.9451 & 0.8896 \\
\hline 40 & flexion & 0.9967 & 0.9946 & 0.9936 & 0.9893 \\
\hline 40 & valgus & 0.9178 & 0.9664 & 0.8424 & 0.9339 \\
\hline 50 & flexion & 0.9888 & 0.9900 & 0.9778 & 0.9803 \\
\hline 50 & valgus & 0.9330 & 0.9239 & 0.8706 & 0.8537 \\
\hline
\end{tabular}

[a]: Subject A

\begin{tabular}{|c|c|c|c|c|c|}
\hline \multirow{2}{*}{$\begin{array}{c}\text { Jump } \\
\text { Ht (cm) }\end{array}$} & \multirow{2}{*}{ irection } & \multicolumn{2}{|c|}{ Correlation Coeff. } & \multicolumn{2}{c|}{ Coeff. Of Determination } \\
\cline { 3 - 6 } & $\mathrm{r}$ (right) & $\mathrm{r}$ (left) & $\mathrm{r}^{2}$ (right) & $\mathrm{r}^{2}$ (left) \\
\hline 30 & flexion & 0.9986 & 0.9973 & 0.9984 & 0.9968 \\
\hline 30 & valgus & 0.9731 & 0.9468 & 0.9844 & 0.9690 \\
\hline 40 & flexion & 0.9821 & 0.9646 & 0.9818 & 0.9639 \\
\hline 40 & valgus & 0.8902 & 0.7925 & 0.8684 & 0.7541 \\
\hline 50 & flexion & 0.9499 & 0.9022 & 0.9544 & 0.9110 \\
\hline 50 & valgus & 0.9244 & 0.8546 & 0.8944 & 0.7999 \\
\hline
\end{tabular}

\section{[b]: Subject B}

Table 11. Pearson correlation coefficient and coefficient of determination for model validation by comparing flexion and valgus joint angles from Inverse Kinematics and Forward Dynamics. [a]: Results from subject A (anonymous) and [b]: Results from subject $B$ (anonymous) executing a perceptively unstable jump with high valgus. 
The table shows that values for $r$ and $r^{2}$ obtained were significantly high. P-values obtained for these correlations were $\mathrm{P}<1 \mathrm{E}-6$ which signify that the validation was successful. These values support the fact that Forward Dynamics analysis used during this study had predicted the joint kinematics correctly.

\subsection{Results from simulations with increased knee valgus}

Simulating effects of added valgus was carried out using the method outlined in Section 3.12. Comparison is made between the simulations, for the case of the $50 \mathrm{~cm}$ jump height, with and without added medial translation induced in the horizontal direction (Figure 19), which was approximately $20-25 \mathrm{~cm}$ depending on individual subjects. Inverse kinematics, computed muscle control, as well as forward dynamic simulations were carried out in OpenSim 2.20. Again, as stated in the beginning of this Chapter, average peaks in text or tables are atemporal averages of individual peaks among eleven subjects, whereas graphs show peaks of temporal averages among all eleven subjects, after positional scaling.

Figures 38 and 39 show temporal average flexion and valgus angles versus percentage time, for the $50 \mathrm{~cm}$ jump simulation with valgus added. Also included in these figures are the simulation results of the original $50 \mathrm{~cm}$ jump for purposes of comparison. 


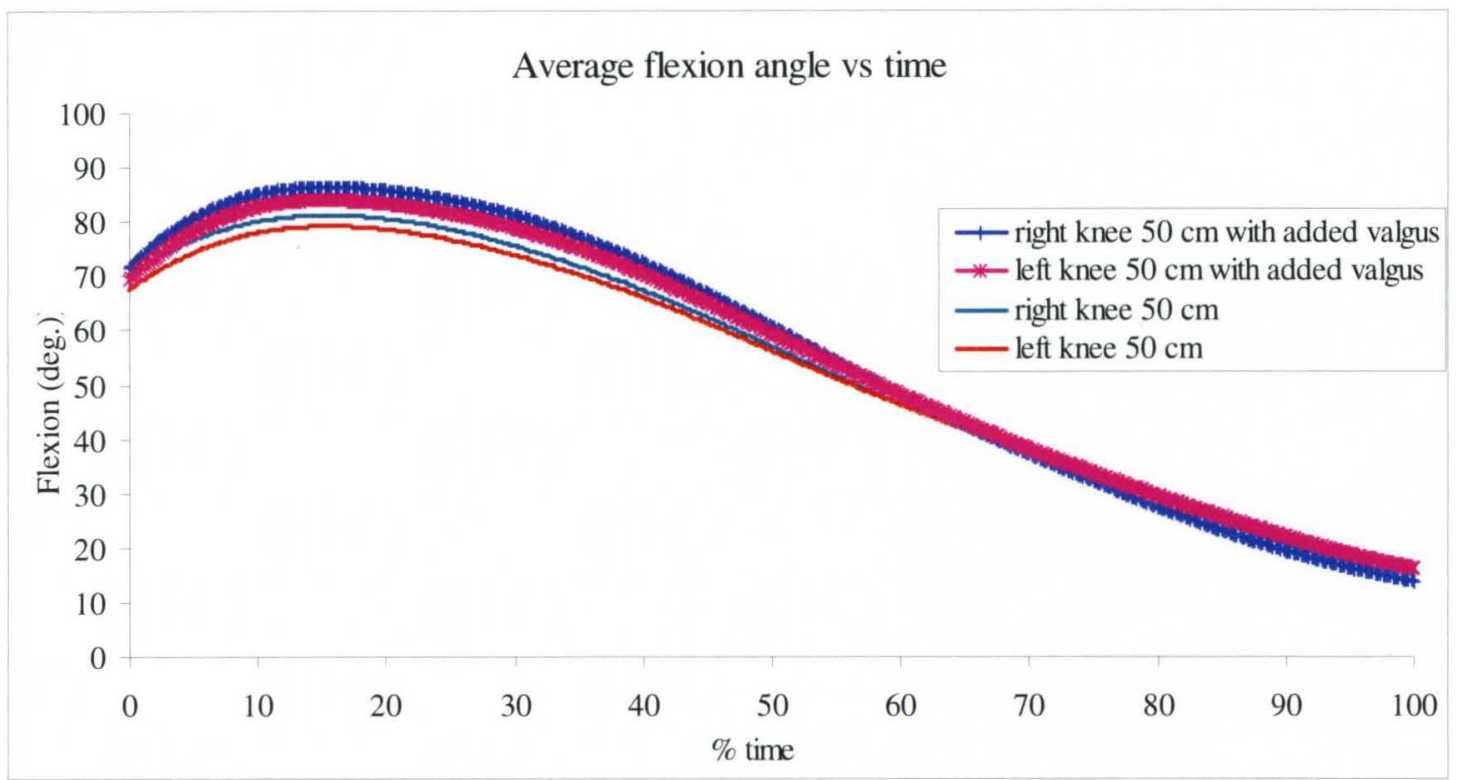

Figure 38. Average right and left knee flexion angles against percentage time obtained from simulations of the original $50 \mathrm{~cm}$ jump and $50 \mathrm{~cm}$ jump with added medial translation (valgus).

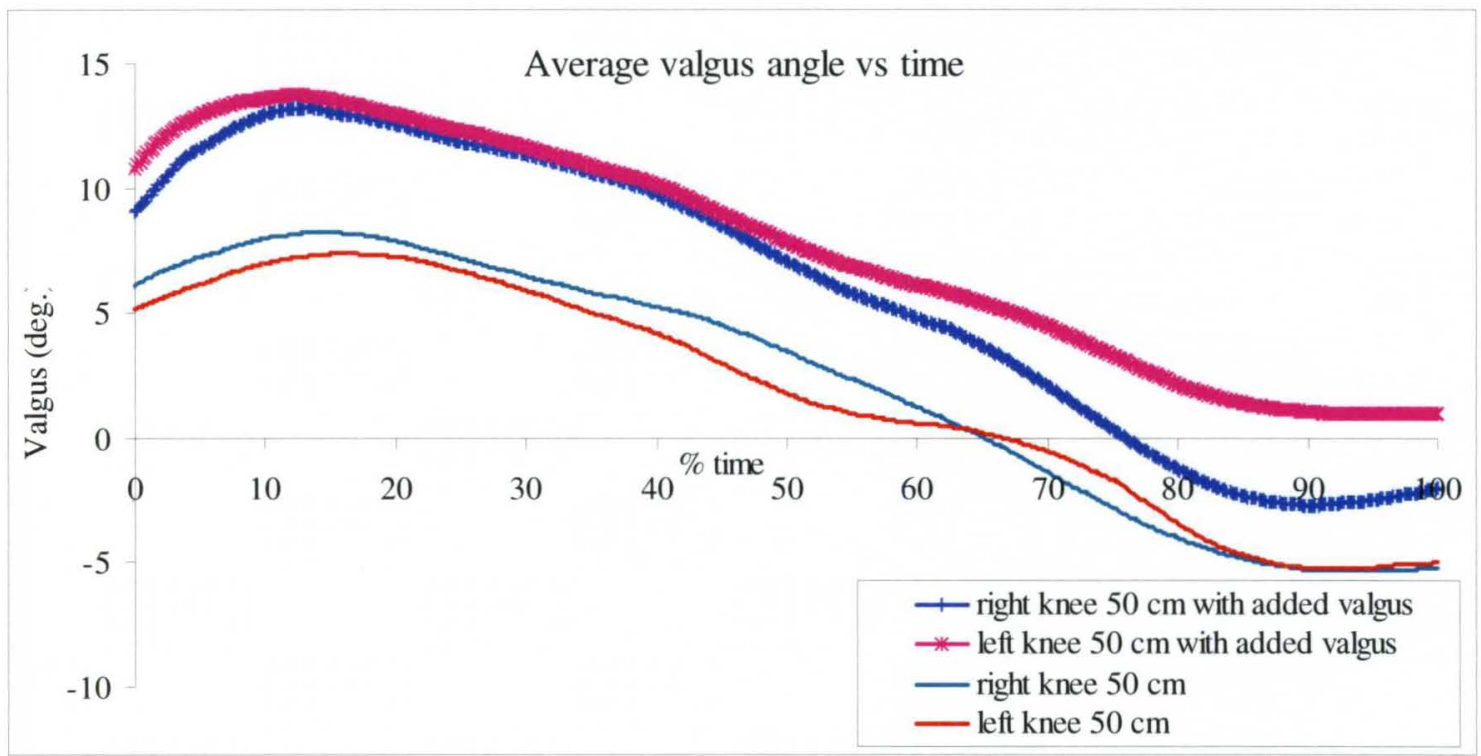

Figure 39. Average right and left knee valgus angles against percentage time obtained from simulations of the original $50 \mathrm{~cm}$ jump and $50 \mathrm{~cm}$ jump with added medial translation (valgus). 
Average peak flexion angles from the simulations with added medial translation were $88.07 \pm 4.71^{\circ}$ and $85.69 \pm 5.17^{\circ}$ for right and left knees respectively. These were significantly higher from the average peak flexion angles of the original $50 \mathrm{~cm}$ jump simulations at $82.0 \pm 6.94^{\circ}$ for right knee and $79.93 \pm 6.67^{\circ}$ for left. Average peak valgus angles for the added medial translation simulations were $13.72 \pm 2.06^{\circ}$ and $14.29 \pm 1.32^{\circ}$ for right and left sides respectively, which were also higher from the average peak valgus angle for the original $50 \mathrm{~cm}$ jump simulations, at $9.92 \pm 1.55^{\circ}$ for right knee and $10.2 \pm$ $1.09^{\circ}$ for the left. This observation is similar to that found by Hewett et al in their 2005 study of vertical drop jumps where they saw increase in peak flexion and valgus angles for ACL injured versus non-injured participants ${ }^{33}$.

Figure 40 shows the temporal averages of $\mathrm{HQ}$ and GQ ratios obtained from the simulated $50 \mathrm{~cm}$ jumps with and without added medial translation. The average peak HQ activation ratios for the added medial translation case were $0.84 \pm 0.06$ for right side and $0.85 \pm$ 0.05 for left, compared to $0.85 \pm 0.07$ for right and $0.81 \pm 0.05$ for left from simulations of the original $50 \mathrm{~cm}$ jumps. The average peak GQ activation ratios for the added medial translation case were $0.63 \pm 0.11$ for right and $0.57 \pm 0.06$ for left compared to $0.61 \pm$ 0.10 for right and $0.57 \pm 0.07$ for left from simulations of the original $50 \mathrm{~cm}$ jump. This implies that flexion-extension muscle activation ratios in the knee joint changed only slightly despite added medial translation. 

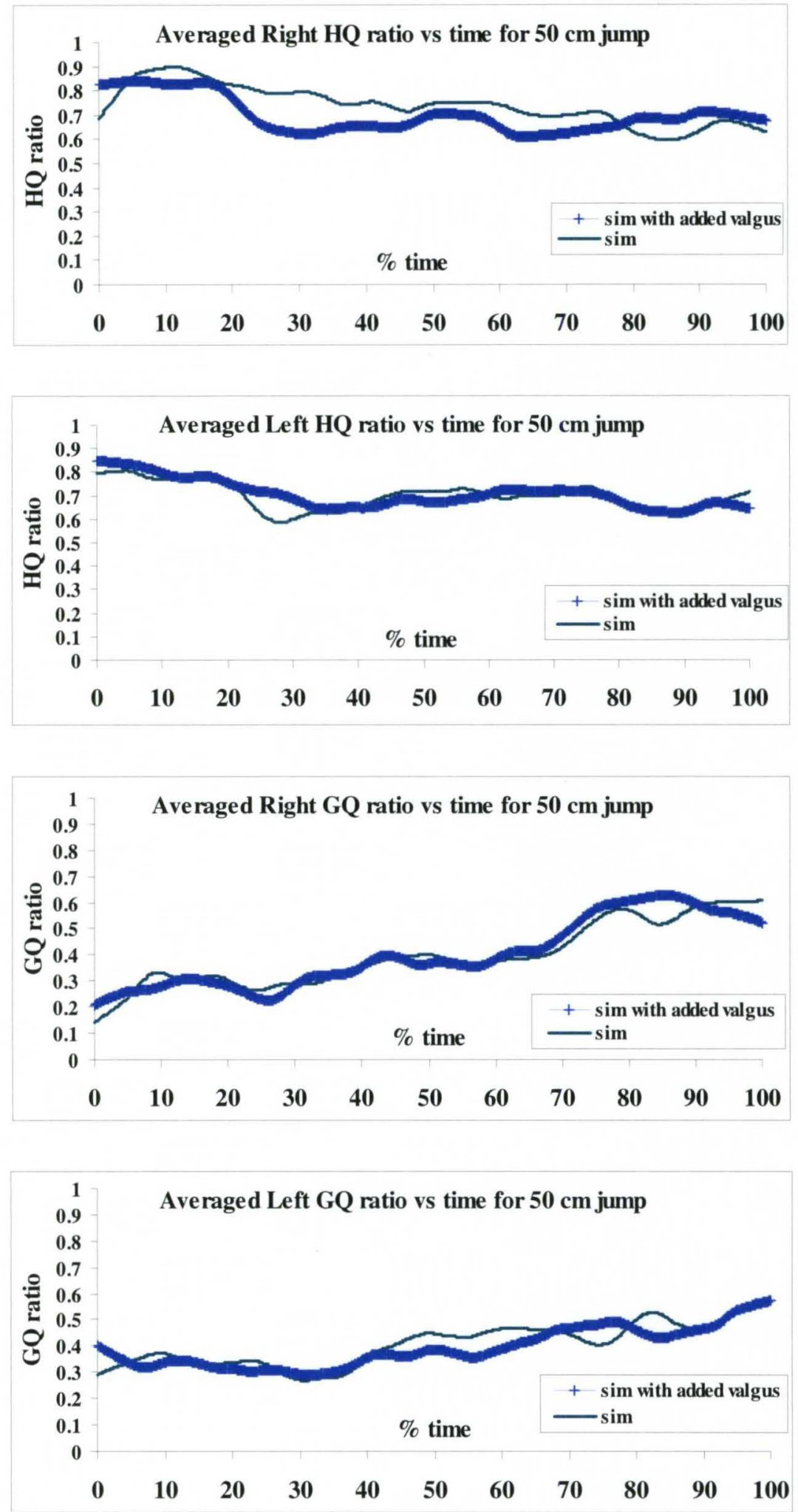

Figure 40. Average simulated $\mathrm{HQ}$ and $\mathrm{GQ}$ ratio from simulations of the original $50 \mathrm{~cm}$ jump and $50 \mathrm{~cm}$ jump with added medial translation (valgus). 
Figures 41-43 shows the effect of increased valgus on ACL fiber length, strain and strain rate. Temporal average ACL fiber lengths and strain increased with added medial translation as shown in Figures 41 and 42, in comparison to the strains obtained from the original $50 \mathrm{~cm}$ simulations. Average peak fiber strain for the added medial translation simulations were $16.27 \pm 5.84 \%$ for right ACL and $15.86 \pm 6.23 \%$ for left. These values were considerably higher from the average peak strains of the original $50 \mathrm{~cm}$ jump simulations which were $13.67 \pm 1.12 \%$ and $13.38 \pm 2.04 \%$ for right and left ACL respectively. Hence it may be concluded that ACL strain increases with valgus. Strain rates in Figure 43 indicate that they went from positive to negative for the added medial translation simulations and behaved similarly to the strain rates from the original $50 \mathrm{~cm}$ jump simulations.

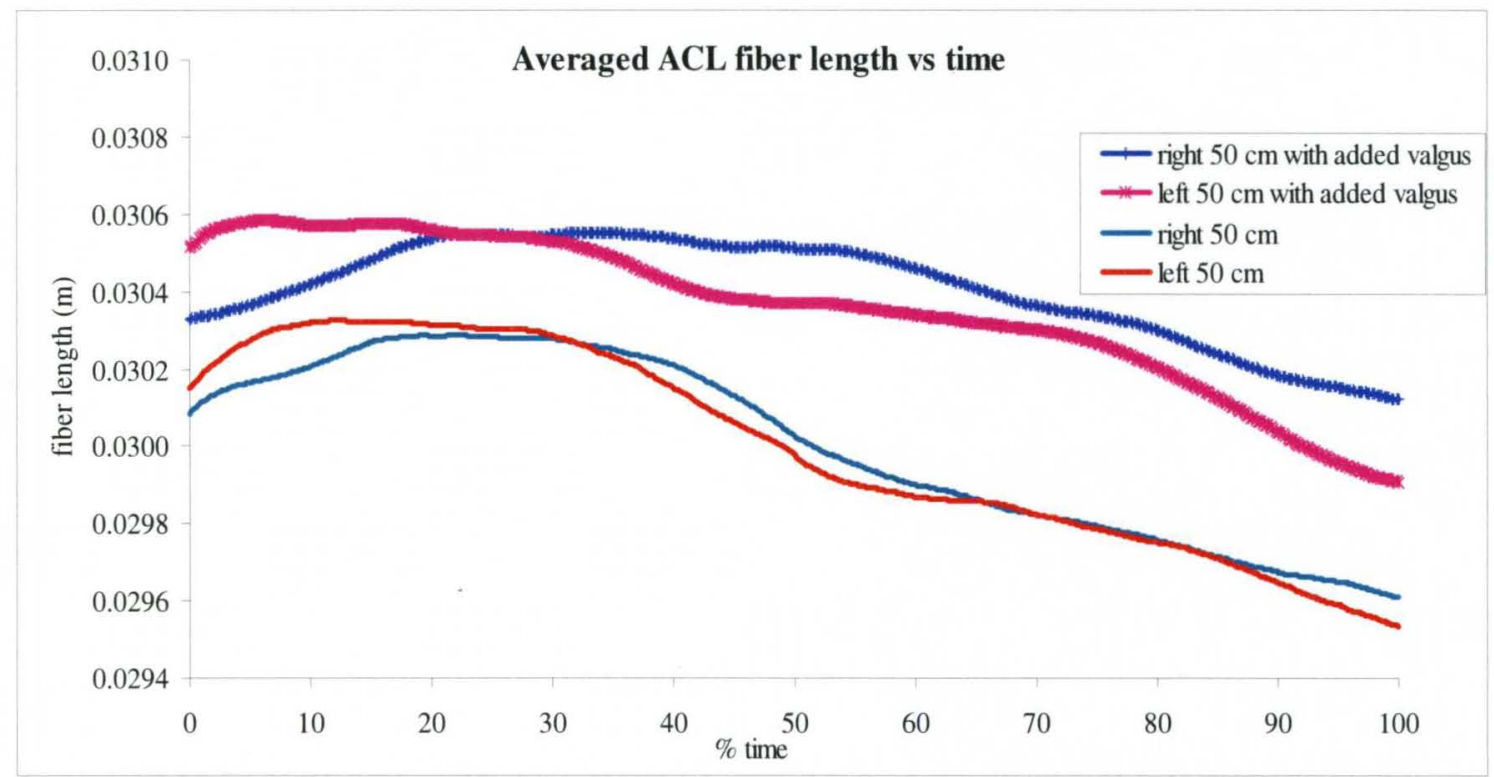

Figure 41. Average right and left ACL fiber length from simulations of the original 50 $\mathrm{cm}$ jump and $50 \mathrm{~cm}$ jump with added medial translation (valgus). 


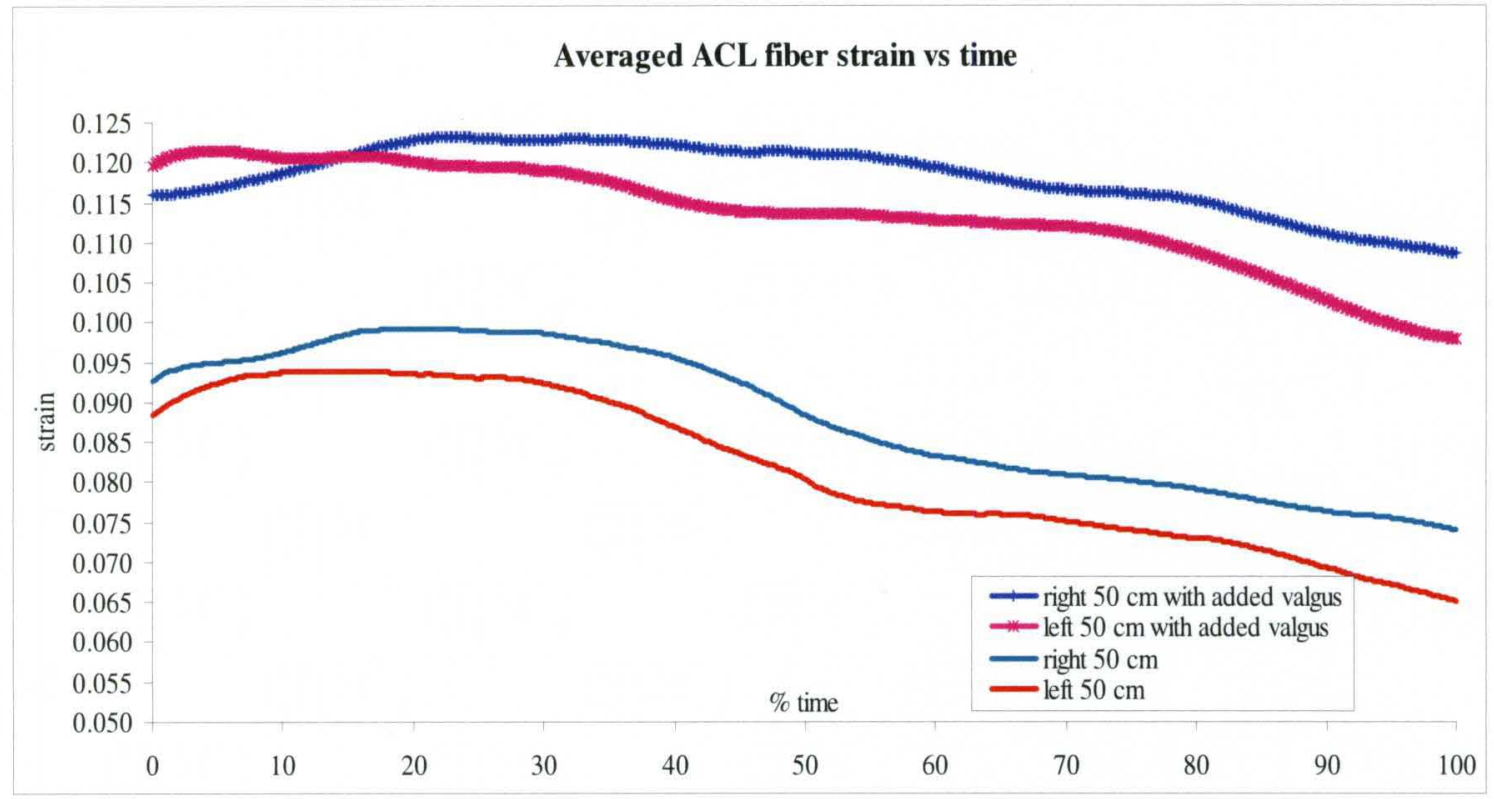

Figure 42. Average right and left ACL fiber strain from simulations of the original 50 $\mathrm{cm}$ jump and $50 \mathrm{~cm}$ jump with added medial translation (valgus).

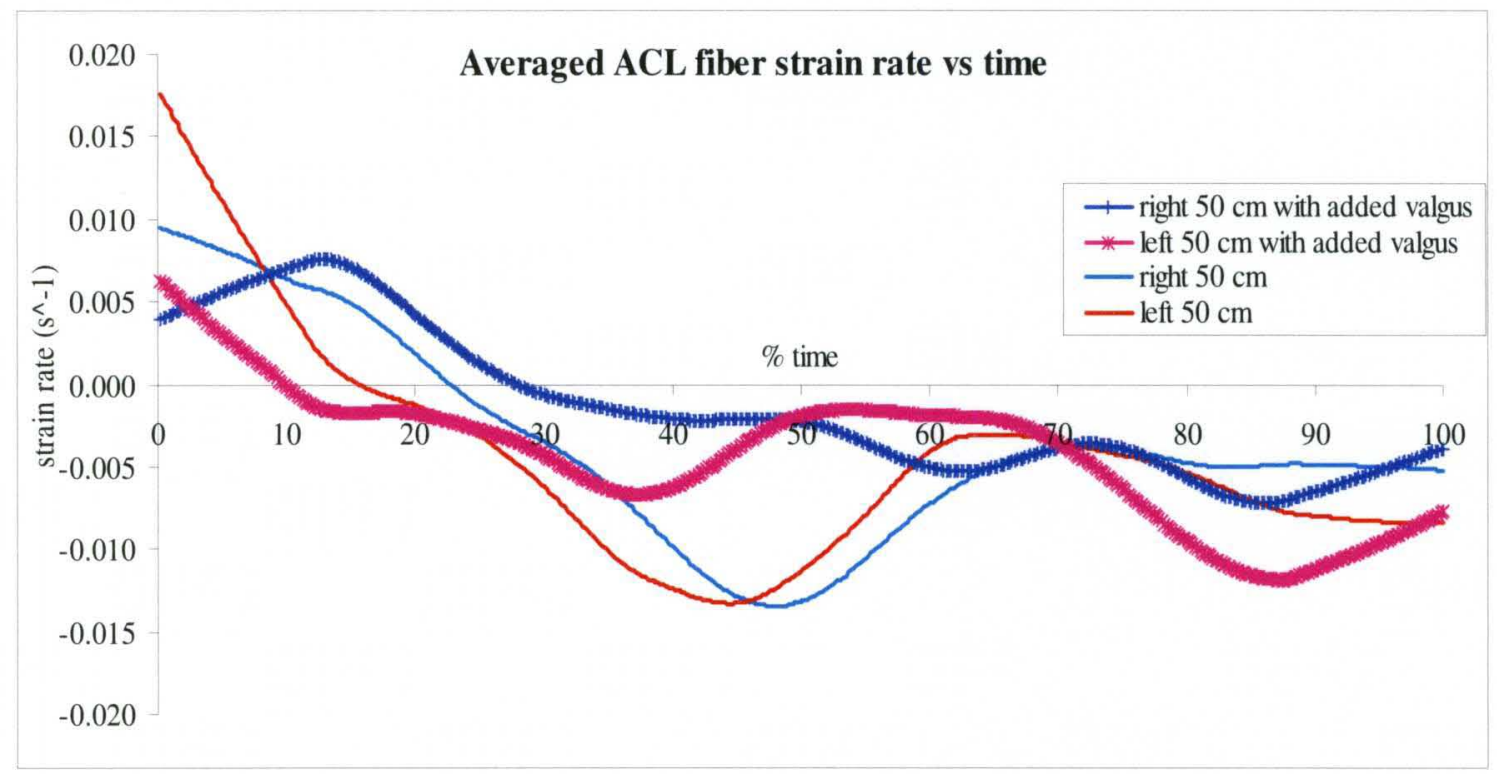

Figure 43. Average right and left ACL fiber strain rate from simulations of the original $50 \mathrm{~cm}$ jump and $50 \mathrm{~cm}$ jump with added medial translation (valgus). 
Temporal average ACL forces from the simulations with and without added medial translation are shown in Figure 44. Average peak fiber force obtained for the added medial translation simulations were $1197.07 \pm 129.11 \mathrm{~N}$ for right $\mathrm{ACL}$ and $1193.11 \pm$ 130.16 $\mathrm{N}$ for left. These values were higher than those from the original $50 \mathrm{~cm}$ jump simulations, which were $1165.36 \pm 123.83 \mathrm{~N}$ for right $\mathrm{ACL}$ and $1160.64 \pm 121.32 \mathrm{~N}$ for left. It is seen that the average ACL forces for the increased valgus simulation were higher than those from the simulation of the original $50 \mathrm{~cm}$ jumps.

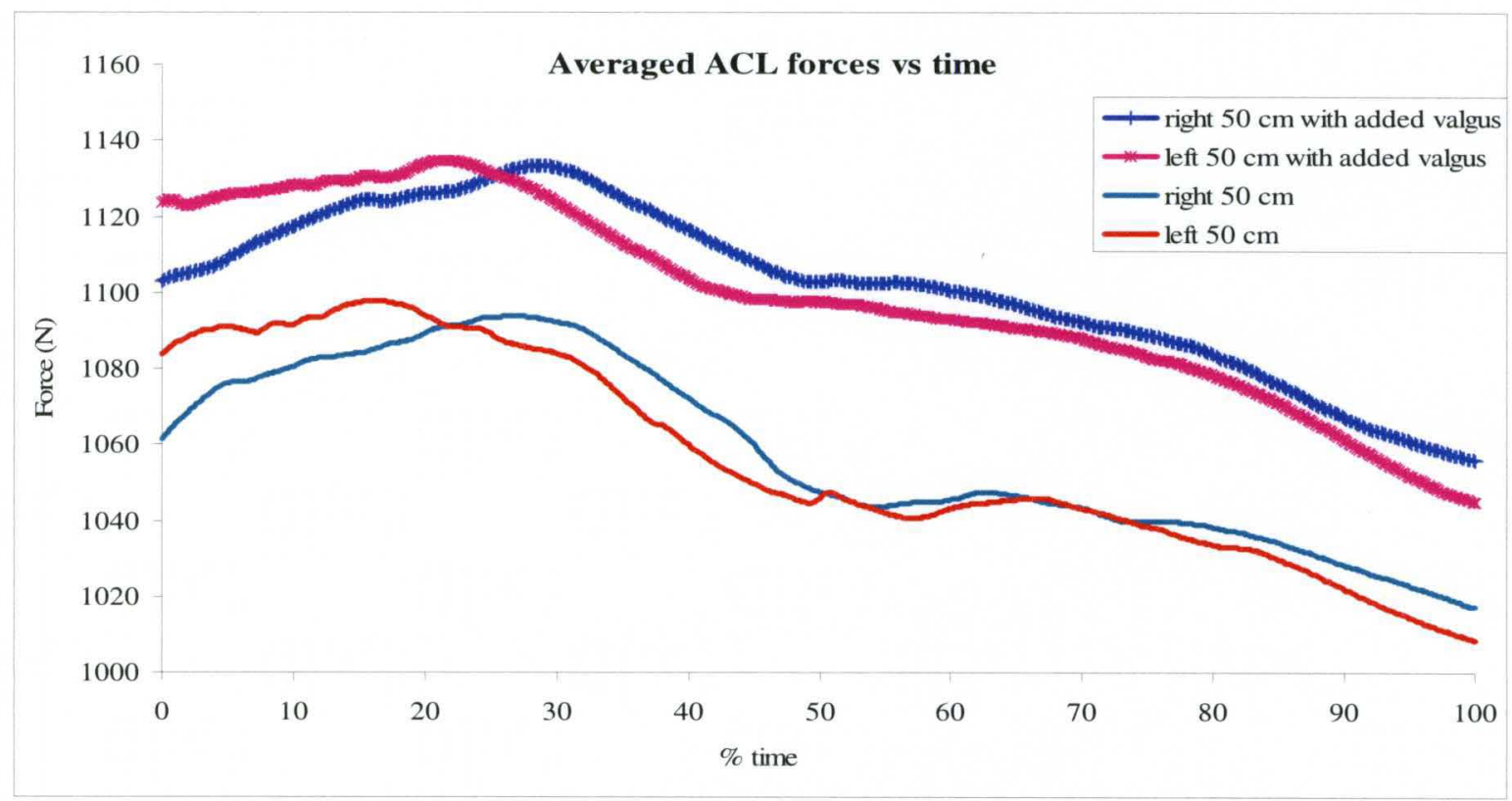

Figure 44. Average right and left ACL fiber forces from simulations of the original 50 $\mathrm{cm}$ jump and $50 \mathrm{~cm}$ jump with added medial translation (valgus). 
Temporal average flexion and valgus knee moments from the $50 \mathrm{~cm}$ jump simulations with and without added medial translation are shown in Figures 45 and 46 . These two figures show that the averaged flexion and valgus moments from the added medial translation simulations did not significantly differ from the averaged flexion and valgus moment from the original $50 \mathrm{~cm}$ jump. Averaged peak magnitude of flexion moments from the simulations with added medial translation were $109.99 \pm 16.97 \mathrm{Nm}$ and $92.11 \pm$ 14.45 $\mathrm{Nm}$ for right and left knees respectively. These were very close to the simulated averaged peak flexion moments at $50 \mathrm{~cm}$ jump height which were $110.00 \pm 17.96 \mathrm{Nm}$ for right knee and $92.11 \pm 14.20 \mathrm{Nm}$ for left. Average peak valgus moments from the simulations with added medial translation were $72.55 \pm 22.42 \mathrm{Nm}$ and $75.63 \pm 20.0 \mathrm{Nm}$ for right and left knees respectively. They did not differ significantly from the averaged peak valgus moments from the original $50 \mathrm{~cm}$ jump simulations which were $72.52 \pm$ $18.75 \mathrm{Nm}$ and $75.15 \pm 28.3 \mathrm{Nm}$ for right and left knees respectively.

In their drop jump study of ACL injured versus non-injured women, Hewett et al., 2009, showed an increase in greater stance phase peak knee valgus moment at the knee for the earlier group, but that was accompanied by increase in GRF forces and moments. However in simulating the drop jumps with added medial translation, knee displacement was the only variable that was changed and no adjustments were made to GRF or GRM. Hence large differences in moments transmitted to the knees were not expected.

Significant correlations were found between knee flexion moments and angles and between knee valgus moments and angles. Correlation coefficient for flexion momentangle pairs were $r=0.93(\mathrm{p}<0.05)$ for right knee and $0.83(\mathrm{p}<0.05)$ for left. The 
correlation coefficient for valgus moment-angle pairs were $0.94(\mathrm{p}<0.05)$ for right knee and $0.93(\mathrm{p}<0.05)$ for left.

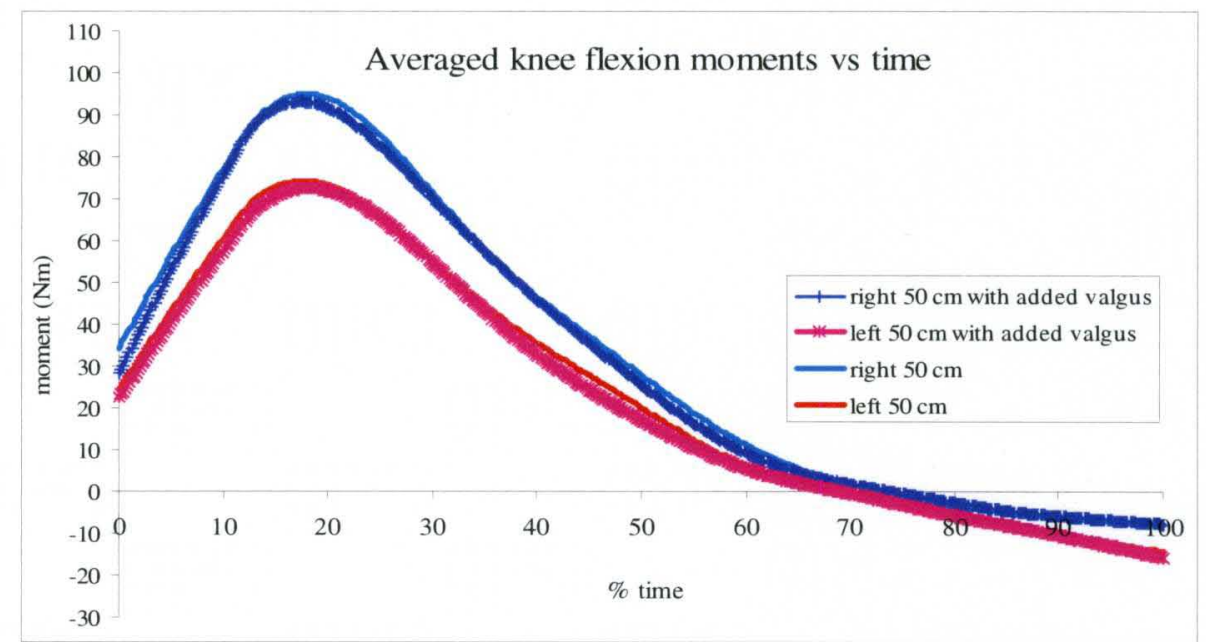

Figure 45. Average right and left flexion knee moments from simulations of the original $50 \mathrm{~cm}$ jump and $50 \mathrm{~cm}$ jump with added medial translation (valgus).

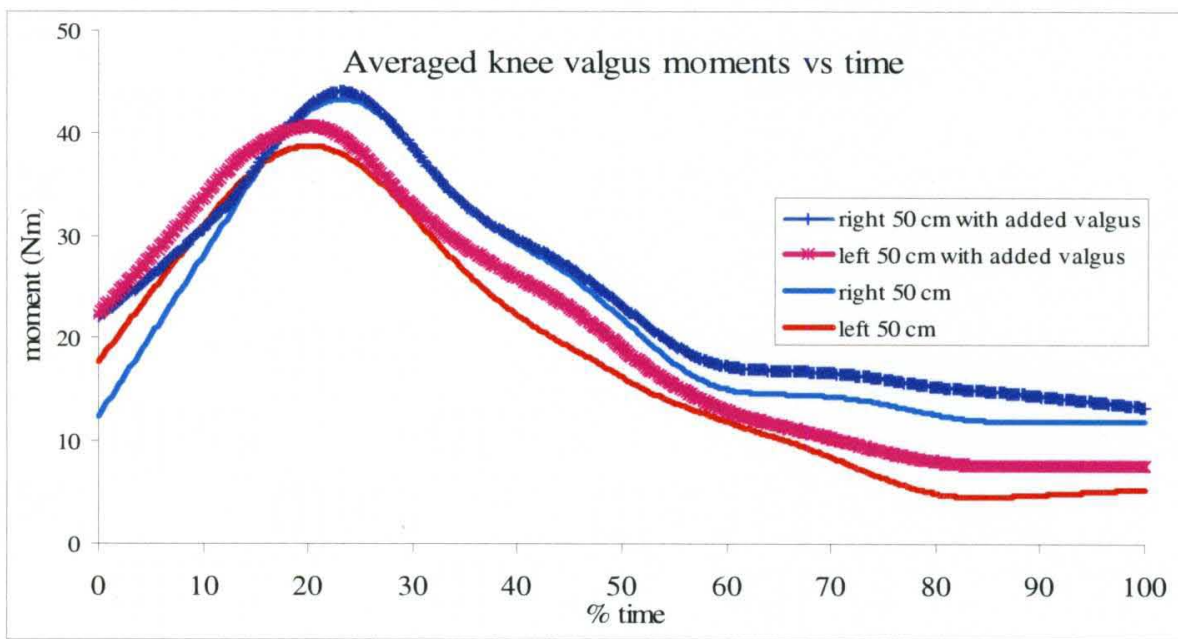

Figure 46. Average right and left knee valgus moments from simulations of the original $50 \mathrm{~cm}$ jump and $50 \mathrm{~cm}$ jump with added medial translation (valgus). 
Figures 47 and 48 shows temporal averages of flexion and valgus knee powers for the added medial translation simulations in comparison to same powers obtained from the original $50 \mathrm{~cm}$ jump simulations. For the added medial translation, average flexion peak power (absorbed) was $436.32 \pm 101.74 \mathrm{~W}$ for right knee and $354.83 \pm 86.61 \mathrm{~W}$ for left. These were in comparison to the original $50 \mathrm{~cm}$ jump simulations where average peak flexion power (absorbed) was $411.93 \pm 57.45 \mathrm{~W}$ and $360.36 \pm 51.71 \mathrm{~W}$ for right and left knee respectively. For added medial translation, average peak valgus power (generated) was $47.15 \pm 23.20 \mathrm{~W}$ for right knee and $50.40 \pm 26.52 \mathrm{~W}$ for left. These were in comparison to the original $50 \mathrm{~cm}$ jump simulations where average peak valgus power (generated) was $61.45 \pm 13.66 \mathrm{~W}$ for the right knee and $41.36 \pm 23.36 \mathrm{~W}$ for the left. No significant trends in knee powers were found for both flexion and valgus due to the above conflicting left and right values.

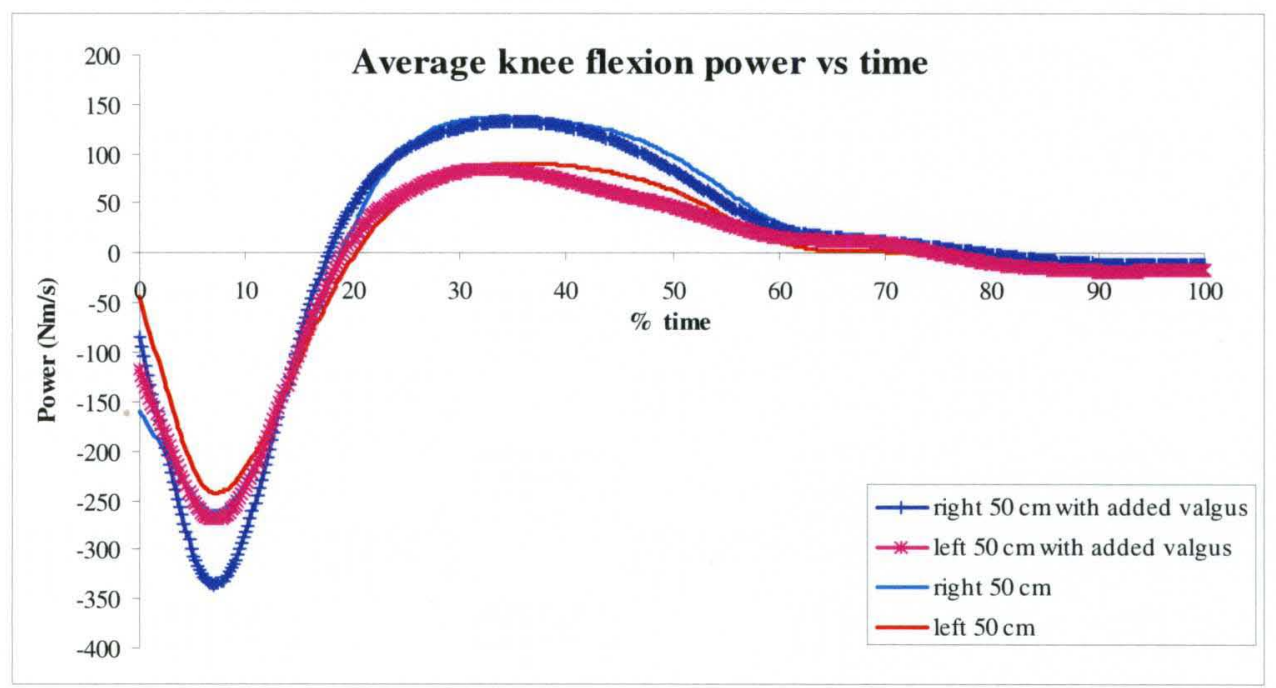

Figure 47. Averaged right and left knee flexion power from simulations of the original $50 \mathrm{~cm}$ jump and $50 \mathrm{~cm}$ jump with added medial translation (valgus). 


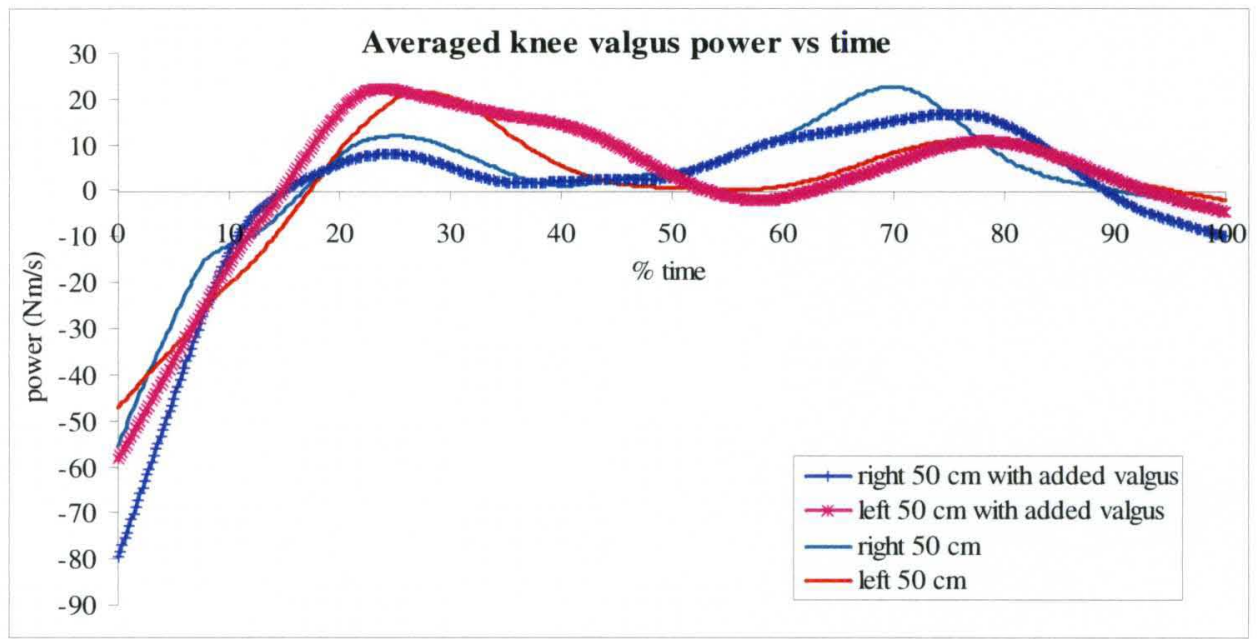

Figure 48. Averaged right and left knee valgus power from simulations of the original $50 \mathrm{~cm}$ jump and $50 \mathrm{~cm}$ jump with added medial translation (valgus).

\subsection{Extended Discussion}

Using available motion capture technologies, the first step of this study was to collect data on recreational female athletes performing drop-jumps from various heights. The research trials conducted within the laboratory gave data on fairly safe drop-jump activities, with low risks of ACL or other injuries. In contrast, real life sporting events involve much higher risks of ACL injury; problems such as severe valgus are not expected to happen during experimental trials, and for obvious reasons, injuries may not be replicated in the laboratory. However it was possible to begin an investigation on ACL injury dynamics by altering some of the variables such as valgus, within the simulation environment used as an investigative tool.

The first research goal of this study was to simulate a complex jump movement consisting of the landing phase of a drop jump. The performance criteria were both 
kinematic and musculoskeletal based. The kinematics controlled the segment and joints positions during motion while $\mathrm{CMC}$ computed the neuromuscular activations causing to attain those positions. Through the final step of forward dynamics, it was found how muscle groups controlled the biomechanical system to perform (the landing phase of) drop-jump movements. Trajectories of the HQ and GQ ratios obtained (Figures 27-29 and 40) gave an optimized pattern of muscle recruitment for the jumps at minimum cost to the neurological system.

Validation tests conducted (section 4.6) showed significant correlations between knee (flexion and valgus) angle values calculated from Inverse Kinematics and Forward Dynamics. Correlations and P-values given in Table 11 indicate that the use of Forward Dynamics for predicting knee joint (motion) angles was justified. Consistently high correlations from the validation tests also meant that if small changes in medial knee translation were introduced for the ensemble of participants, Forward Dynamics simulation would yield reliable results.

The second research goal was to determine the effects of increasing valgus alignment of the knee on ACL loads and strains. Not surprisingly, substantial increases in ACL loads and strains were found (Section 4.7), a phenomenon long suspected by bio-mechanical research communities. No significant difference in muscle activation patterns, the HQ and GQ ratios (Figure 40), were seen in the additional valgus simulations. A strong argument for observing little changes in HQ and GQ ratios is that the hamstring, quadriceps and gastrocnemius are all flexor-extensor muscles, their control having little 
to do with abduction movement. Therefore, etiological studies with different muscle groups (from the above) will need to be conducted to find the location of muscular control influencing excess valgus.

Once the second research goal was reached, the results showed the effects of increased valgus on ACL strains and forces. The simulations showed that ACL strain increased from $13.67 \%$ to $16.27 \%$ for right ACL and $13.38 \%$ to $15.86 \%$ for left. This increase puts the ACL in danger of rupture according to Butler's study which claimed that no more than $13-14 \%$ strain for the ACL was safe ${ }^{9}$. Along with strain, there was increase in the ACL force too for the added medial translation situation as seen in Section 4.7. This increase in strain of and force on the ACL with added medial translation (during simulation) is considered the most important finding of this study. This inference can be compared favorably to Solomonow's study ${ }^{65,24}$.

Various literatures on ACL injury demonstrate that many physical and psychological variables affect injury to the ACL. It is likely that some of the dynamic factors affecting ACL injury has not been discovered and evaluated. In the present study several factors contributing to knee motion, including other knee muscles and also knee ligaments like PCL, MCL and LCL, were left out. However, it may be concluded that excessive valgus of the knee is a major contributor to ACL injury. As more variables of risk are discovered it would be advantageous to establish some form of screening program that will help towards preconditioning athletes against ACL injury. Formulation of an effective screening program for testing risks of ACL injury is a challenging issue. As Barber- 
Westin et al., 2010, points out, drop jump tests conducted within the laboratory are not good indicators of ACL injury risks ${ }^{6}$. In the absence of other procedures, at present this is the only safe method available to research. Enhancing the analysis by using a more comprehensive musculoskeletal model (something as yet unprecedented) complete with lower extremity ligaments will more than likely move the understanding real time ACL mechanics further ahead.

The most original part of this study was the inclusion of ACL in simulations. Ability to trace length and force changes in the ACL during impact was highly informative. Added to this was ability to visualize dynamic changes in the ACL through OpenSim visual interface. However there were some obvious limitations to the musculoskeletal model developed, which was rather simplified. The primary limitation was lack of muscle volume. Representation of muscles (and ACL) as line segments with constant cross section area was possibly the greatest factor contributing towards variation of simulation results from real life. A second limitation was obtaining muscle activation based on isometric force at an ideal muscle-tendon isometric condition. However in everyday activities, including jumping, chances of muscles reaching MVC are seldom. Hence at certain times during simulation, the musculoskeletal model underwent activations equal to one due to a muscle excited at peak isometric force. 


\section{CHAPTER V}

\section{CONCLUSION}

The purpose of the present study was to investigate the dynamic variables of the knee and ACL during drop jump trials and to observe effects of increased valgus on the ACL. Both investigations have been carried out successfully with clear results presented while maintaining high standards of scientific enquiry.

Drop-jump results from the three different heights showed distinct trends, such as, ACL strains and loads increased with increased jump height. Knee flexion angles, knee flexion moments and valgus angles increased with increased drop jump height. No definitive conclusions were drawn between knee valgus moment, and ACL and Knee Biomechanics with increased medial translations.

Distinct trends were also observed from the simulations with increased medial translation, e.g., increase in ACL strain and load as well as valgus angle. There were negligible changes in knee moment, possibly due to unchanged GRF force and short ACL moment arm. 


\section{CHAPTER VI}

\section{FUTURE DIRECTIONS}

Better understanding of ACL dynamics may be achieved through simulation with enhanced anatomical model of the knee and lower extremities. A better physiologically realistic representation of muscles including emphasis on underlying fibril-collagen microstructure is very important. Modeling of bone structures and functionalities may also be improved. For example there needs to be a provision for calculating bone-to-bone contact forces. A more comprehensive picture of the knee joint, complete with ACL, PCL and MCL ligaments, will provide further insight into real life ACL load and strain.

ACL dynamics needs to be investigated for parameters, as yet unknown, contributing to risk of injury. Sophisticated EMG systems that capture the activation in hard-to-reach muscles in a non-invasive way need to be designed. Studies need to be done on athletes who are back into their athletic programs after rehabilitation from ACL injury or reconstruction. Effects of increased GRF on the increased valgus situation would provide insight into a more serious injury condition. Variations in local loads at the knee joint, especially increase in abduction (valgus) moment needs to be examined. Finally, as other risk factors are discovered and their patterns are understood, preventive measures may be formulated, on an individual basis, to minimize (may be even avoid) ACL injuries. 


\section{REFERENCES}

1. Anderson F.C., Goldberg S.R., Pandy M.G., Delp, S.L., Contributions of muscle forces and toe-off kinematics to peak knee flexion during the swing phase of normal gait: an induced position analysis. Journal of Biomechanics. 2004; 37: 731-737.

2. Anderson F.C., Pandy M.G., A dynamic optimization solution for vertical jumping in three dimensions. Computer Methods in Biomechanics and Biomedical Engineering 1992; 201-231.

3. Anderson, F.C., Pandy, M.G., Individual muscle contributions to support in normal walking. Gait \& Posture. 2003; 17: 159-169.

4. Anderson F.C., Pandy M.G., Dynamic optimization of human walking. Journal of Biomechanical Engineering. 2001; 123: 381-390.

5. Badler N.I., Smoliar S.W., Digital representations of human movement. ACM Comput. Surv. 1979; 11(1): 19-38.

6. Barber-Westin S.D., Smith S.T., Campbell T., Noyes F.R., The drop-jump video screening test: Retention of improvement in neuromuscular control in female volleyball players Cincinnati Sports medicine Research and Education Foundation, Cincinnati, Ohio. 2010; 24(11): 3055-3062.

7. Bauby C.E., Kuo A.D., Active control of lateral balance in human walking. Journal of Biomechanics. 2000; 33: 1433-1440.

8. Burkhardt E., Barton B., Grahammer. Maximum impact and propulsion forces during jumping and explosive lifting exercises. J of Applied Sports Science Research. 2006; 4(3): 107-117.

9. Butler D.L., Kay M.D., Stouffer D.C., Comparison of material properties in fascicle bone units from human patellar tendon and knee ligaments. Journal of Biomechanics. 2003; 19: 425-432.

10. Cappozzo A., Catani F., Croce U.D., Leardini A., Position and orientation in space of bones during movement: anatomical frame definition and determination. Clinical Biomechanics (Bristol, Avon). 1995; 10(4): 171-178. 
11. Carhart M.R., Biomechanical Analysis of Compensatory Stepping: Implications for Paraplegics Standing Via FNS. Department of Bioengineering, Arizona State University, Tempe. 2000.

12. Chappell J.D., Yu, B., Kirkendall D.T., Garrett, W.E., A comparison of knee kinetics between male and female recreational athletes in stop-jump tasks. Am. J. Sports Med. 2002; 30(2): 261-267.

13. Cram J.R., Kasman G.S., Introduction to Surface Electromyography. Aspen Publishers, Gaithersburg, MD, 1998; 368-373.

14. Dapena J., Harman E.A., Miller J.A., Three-dimensional cinematography with control object of unknown shape. J. Biomech.1982; 15: 11-19.

15. Delp S.L., Blemker S.S., Three-dimensional representation of complex muscle architectures and geometries. Annals of Biomedical Engineering. 2005. 33(5): 661-673.

16. Delp S.L., Thelen D.G., Anderson F.C., Generating dynamic simulations of movement using computed muscle control. Journal of Biomechanics. 2003; 36(19): 321-328.

17. Delp S.L., Loan J.P., A computational framework for simulating and analyzing human and animal movement. IEEE. Computing in Science and Engineering. $2000 ; 46: 57$.

18. Delp S.L. and Loan J.P., A graphics-based software system to develop and analyze models of musculoskeletal structures. Comput.Biol. Med. 1995; 25: 2134.

19. Delp S.L., Maloney W., Effects of hip center location on the moment-generating capacity of the muscles. J. Biomech. 1993; 26: 485-499.

20. Delp S.L., Loan J.P., Hoy M.G., Zajac F.E., Topp E.L. Rosen J.M., An interactive graphics-based model of the lower extremity to study orthopaedic surgical procedures. IEEE Transactions on Biomedical Engineering. 1990; 39: 1107-1115.

21. Delp S.L., Thelen D.G., Anderson, F.C., Using computed muscle control for generating forward dynamic simulation of human walking from experimental data. Journal of Biomechanics. 2006; 36(19): 321-328.

22. DeLuca C.J., Surface Electromyography: Detection and Recording. Delsys Incorporated. 2002. 
23. Epstein M., Herzog W., Theoretical Models of Skeletal Muscle. Wiley, Chichester. 1998.

24. Eversull E., Solomonow M., Zhou B., Baratta R.V., Zhu M., Neuromuscular neutral zones sensitivity to lumbar displacement rate, Clin.Biomechanics. 2001; 16: $102-113$.

25. Fernandez J.W., Pandy M.G., Integrating modelling and experiments to assess dynamic musculoskeletal function in humans. Experimental Physiolog. 2006; 91 : 371-382.

26. Gene D.M., Weinhold P., Blackburn T., Chudik S., Garrett W., Aggressive Quadriceps Loading Can Induce Noncontact Anterior Cruciate Ligament Injury. Am. J. Sports Med. 2004; 32: 477-483.

27. Fernandez J.W., Pandy M.G., Integrating modelling and experiments to assess dynamic musculoskeletal function in humans. Experimental Physiolog. 2006; 91 : $371-382$.

28. Gao F., Damsgaard M., Rasmussen J., Christensen S.T., Computational method for muscle-path representation in musculoskeletal models. Biolog. Cybern. 2002; 87: 199-210.

29. Garner B.A., Pandy M.G., The obstacle-set method for representing muscle paths in musculoskeletal models. Comput. Methods Biomech. Biodmed. Eng. 2000; 3: $1-30$.

30. Griffin L.Y., Noncontact Anterior Cruciate Ligament Injuries: Risk Factors and Prevention Strategies. Journal of the American Academy of Orthopaedic Surgeons. 2000; 8: 141-150.

31. Guoan L., DeFrate M.D., Rubash H.E., Gill T.J., In vivo kinematics of the ACL during weight-bearing knee flexion. Journal of Orthopaedic Research. 2005; 23: $340-344$.

32. Hatze H., High-precision three-dimensional photogrammetric calibration and object space reconstruction using a modified DLT-approach. J. Biomech. 1988; 21: 53-58.

33. Hewett T.E., Myer G.D., Ford K.R., Heidt R.S. Jr, Colosimo A.J., McLean S.G., Biomechanical Measures of Neuromuscular Control and Valgus Loading of the Knee Predict Anterior Cruciate Ligament Injury Risk in Female Athletes: A Prospective Study. Am. J. Sports Med. 2005; 33: 492-501.

34. Hewett T.E., Lindenfeld T.N., Riccobene J.V., Noyes F.R., The effect of neuromuscular training on the incidence of knee injury in female athletes: a prospective study. Am J Sports Med. 1999; 27: 699-706. 
35. Hewett T.E., Paterno M.V., Myer G.D., Strategies for enhancing pro-prioception and neuromuscular control of the knee. Clin Orthop. 2002; 402:76-94.

36. Hewett T.E., Myer G.D., Ford K.R., Anterior cruciate ligament injuries in female athletes: Part 1, mechanisms and risk factors. American Journal of Sports Medicine. 2006; 34(2): 299-311.

37. Hill, AV., The heat of shortening and the dynamic constants of muscle. Proc. R. Soc. London. 1938; 126: 136-95.

38. Hollister A.M., Jatana S., Singh A.K., Sullivan W.W., Lupichuk A.G., The axes of rotation of the knee. Clinical Orthopaedics and Related Research. 1993; 290: 259-268.

39. Jonsson H., Karrholm J., Three-dimensional knee joint movements during a stepup. Evaluation after anterior cruciate ligament rupture. Journal of Orthopaedic Research. 1994; 12: 769-779.

40. Kastelic J., Galeski A., Baer E., The multicomposite structure of ligaments. Connective Tissue Research. 1978; 6: 11-23.

41. Katz B., The relation between force and speed in muscular contraction. J. Phys. 1939; 96: 45-64.

42. Krosshaug T., Nakamae A., Boden B.P., Engebretsen L., Smith G., Slauterbeck J.R., Hewett T.E., Mechanisms of Anterior Cruciate Ligament Injury in Basketball: Video Analysis of 39 Cases. Am. J. Sports Med. 2007; 35: 359-367.

43. McLean S.G., Su A., van den Bogert, A.J., Development and validation of a 3-D model to predict knee joint loading during dynamic movement. J. Biomech. Eng. 2003; 125: 864-873.

44. McLean, S., Huang, X., van de Bogert, T., Sagittal plane biomechanics during sport movements does not explain higher incidence of ACL injury in females. In: Proceedings of the ISB $20^{\text {th }}$. 2005. Congress-ASB 29th Annual Meeting, Cleveland, $\mathrm{OH}$.

45. McNair P.J., Marshell R.N., Matheson J.A., Important features associated with anterior cruciate ligament injury. New Zeal. Med. J. 1991; 103: 537-539.

46. Miller N.R., Shapiro R., McLaughlin T.M., A technique for obtaining spatial kinematic parameters of segments of biomechanical systems from cinematographic data. J. Biomech. 1980; 13: 535-547. 
47. Myer G.D., Ford K.R., McLean S.G., Hewett T.E., The Effects of Plyometric Versus Dynamic Stabilization and Balance Training on Lower Extremity Biomechanics. Am. J. Sports Med. 2006; 34: 445-455.

48. National Federation of State High School Associations. 2002 High School Participation Survey. Indianapolis, Ind: National Federation of State High School Associations. 2002.

49. Neptune R.R., Optimization algorithm performance in determining optimal controls in human movement analyses. Journal of Biomechanical Engineering. 1999; 121: 249-252.

50. Neptune R.R., Hull M.L., Evaluation of performance criteria for simulation of submaximal steady-state cycling using a forward dynamic model. Journal of Biomechanical Engineering. 1998; 120: 334-341.

51. Neptune R.R., Kautz S.A., Zajac F.E., Contributions of the individual ankle plantar flexors to support, forward progression and swing initiation during walking. Journal of Biomechanics. 2001; 34: 1387-1398.

52. Neptune R.R., Zajac F.E., Kautz S.A., Muscle force redistributes segmental power for body progression during walking. Gait \& Posture. 2004; 19: 194-205.

53. Palmieri-Smith R.M., Wojtys E.M., Ashton-Miller J.A., Association between preparatory muscle activation and peak valgus knee angle. Journal of Electromyography and Kinesiology. 2008; 18: 973-979.

54. Pandy M.G., Computer modeling and simulation of human movement. Ann. Rev. Biomed. Eng. 2001; 3: 245-273.

55. Pandy M.G., Anderson F.C., Hull D.G., A parameter optimization approach for the optimal control of large-scale musculoskeletal systems. ASME Journal of Biomechanical Engineering. 1992; 114(4): 343-363.

56. Pandy M.G., Computer modeling and simulation of human movement. Annual Review of Biomedical Engineering. 1990; 3: 245-273.

57. Pandy M.G., Zajac F.E., Sim E., Levine W.S., An optimal control model for maximum-height human jumping. Journal of Biomechanics. 2001; 23(12): 11851198.

58. Rassier D.E., Lee E.J., Herzog W., Modulation of passive force in single skeletal muscle fibres. Biology Letters. 2005; 1(3): 342-345. 
59. Renstrom P., Arms S.W., Stanwyck T.S., Johnson R.J., Pope M.H., Strain within the anterior cruciate ligament during hamstring and quadriceps activity. Am J Sports Med. 1986; 14: 83-87.

60. Rodgers J.L., Nicewander W.A., Thirteen ways to look at the correlation coefficient. The American Statistician. 1988; 42: 59-66.

61. Shelburne K.B., Pandy M.G., A musculoskeletal model of the knee for evaluating ligament forces during isometric contractions. J. Biomech. 1997; 30: 163-176.

62. Shin C.S., Chaudhari A.M., Andriacchi T.P., The influence of deceleration forces on ACL strain during single-leg landing: a simulation study. J. Biomech. 2007; 40 (5): 1145-1152.

63. Shoemaker S.C., Markolf K.L., The role of the meniscus in anterior-posterior stability. J. Bone Joint Surg. 1986; 71-79.

64. Simbios-OpenSim. Version 2.2.0. Department of Biomedical Engineering, Stanford University. C 2010.

65. Solomonow M., Ligaments: a source of work-related musculoskeletal disorders. Journal of Electromyography and Kinesiology. 2004; 14: 49-60.

66. Spoor C.W., Veldpaus F.E., Rigid body motion calculated from spatial coordinates of markers. J Biomech.1980; 13(4): 391-393.

67. Stauber W.T., Eccentric action of muscles: Physiology, injury and adaptation. Exerc Sport Sci Rev. 1989; 17: 157-185.

68. Stern J.T., Computer modelling of gross muscle dynamics. Journal of biomechanics. 1974; 7(5): 411-28.

69. Tang C., Zhang G., Tsui C., A 3d skeletal muscle model coupled with active contraction of muscle fibres and hyperelastic behaviour. Journal of biomechanics. $2009 ; 42(7)$ : 865-872.

70. Teitz CC., Video analysis of ACL injuries. In: Griffin LY, ed. Prevention of Noncontact ACL Injuries. Rosemont, Ill: American Association of Orthopaedic Surgeons. 2001; 87-92.

71. Teran J., Blemker S., Hing V.N.T., Fedkiw R., Finite volume methods for the simulation of skeletal muscle. In SCA '03: Proceedings of the 2003 ACM SIGGRAPH/Eurographics symposium on Computer animation. 2003; 68-74. 
72. Thelen D.G., Anderson FC., An operational space tracking algorithm for generating dynamic simulations of movement. Computer Methods in Biomechanics and Biomedical Engineering, November. 2001; Rome, Italy.

73. Thelen D.G., Anderson F.C., Using computed muscle control to generate forward dynamic simulations of human walking from experimental data. Journal of Biomechanics. 2006; 39: 1107-1115.

74. Vaughan C.L., Andrews J.G., Hay J.G., Selection of body segment parameters by optimization methods. Journal of Biomechanical Engineering. 1982; 104:38-44.

75. Winter D.A., Energy generation and absorption at the ankle and knee during fast, natural and slow cadences. Clin. Orthop. Rel. Res. 1983; 175: 147-154.

76. Winter D.A., Biomechanics and Motor Control of Human Movement (3/e). Wiley Int. 1990; New York, USA.

77. Winter DA. Biomechanical motor patterns in normal walking. J. Motor Behavior. 1980; 15: 302-330.

78. Withrow T.J., Huston L.J., Wojtys E.M., Ashton-Miller J.A., The relationship between quadriceps muscle force, knee flexion, and anterior cruciate ligament strain in an in vitro simulated jump landing. Am J Sports Med. 2006; 34: 269-274.

79. Wojtys E.M., Ashton-Miller J.A., Huston L.J., A gender-related difference in the contribution of the knee musculature to sagittal-plane shear stiffness in subjects with similar knee laxity. J Bone Joint Surg Am. 2002; 84: 10-16.

80. Wojtys E.M., Huston L.J., Schock H.J., Boylan J.P., Ashton-Miller J.A., Gender differences in muscular protection of the knee in torsion in size-matched athletes. J Bone Joint Surg Am. 2003; 85: 782-789.

81. Zatriosky V.M., Biomechanics in Sports: Performance enhancement and injury prevention. IOC Medical Comission, International Federation of Sports Medicine. Blackwell Science Ltd. 2000. Oxford, UK. 


\section{APPENDICES}

\section{APPENDIX - A}

\section{Human Movement Glossary}

Flexion - Bending movement that decreases the angle between two parts. Bending the elbow, clenching a hand into a fist and folding the knee when sitting are examples of flexion. Flexion of the hip or shoulder moves a limb forward, towards the anterior side of the body.

Extension - It is the opposite of flexion, being a straightening movement that increases the angle between body parts. When standing up, the knees are extended. Extension of the hip or shoulder moves a limb back, towards the posterior side of the body.

Abduction - A motion that pulls a structure or part away from the midline of the body. In the case of fingers and toes, abduction happens with spreading the digits apart away from the centerline of the hand or foot.

Adduction - A motion that pulls a structure or part towards the midline of the body, or towards the midline of a limb. Dropping the arms to the sides and bringing the knees together are examples of adduction. In the case of the fingers or toes, adduction is closing the digits together. 
Internal rotation - Also known as medial rotation of the shoulder or hip which would point the toes and knees or the flexed forearm inwards (towards the midline).

External rotation - Also known as lateral rotation is the opposite of the above. It would turn the toes and knees or the flexed forearm outwards (away from the midline).

Dorsiflexion - Flexion of the entire foot superiorly, like taking the foot off an automobile pedal.

Plantarflexion - Flexion of the entire foot inferiorly, as if pressing an automobile pedal.

Eversion - Movement of the sole of the foot away from the median plane.

Inversion - Movement of the sole towards the median plane which is the same as twisting an ankle. 


\section{APPENDIX - B}

\section{Hill-Huxley force-length-velocity surface for finding active muscle forces}

Figure 49 shows the ideal force-length-velocity curve from Katz et al. ${ }^{20}$. The investigation on force-length and force-velocity relationship in muscles was first started by Fenn and Marsh (1935), A.V. Hill (1938) and Katz (1939). Katz was one of the first to perform force-velocity experiments with both concentrically (positive velocity) and eccentrically (negative velocity) muscles. When eccentric contractions occur the fibers are essentially lengthening which implies negative velocity. Katz also noted that eccentric contractions occurred when a muscles was yielding to a force and vice versa for concentric contraction.

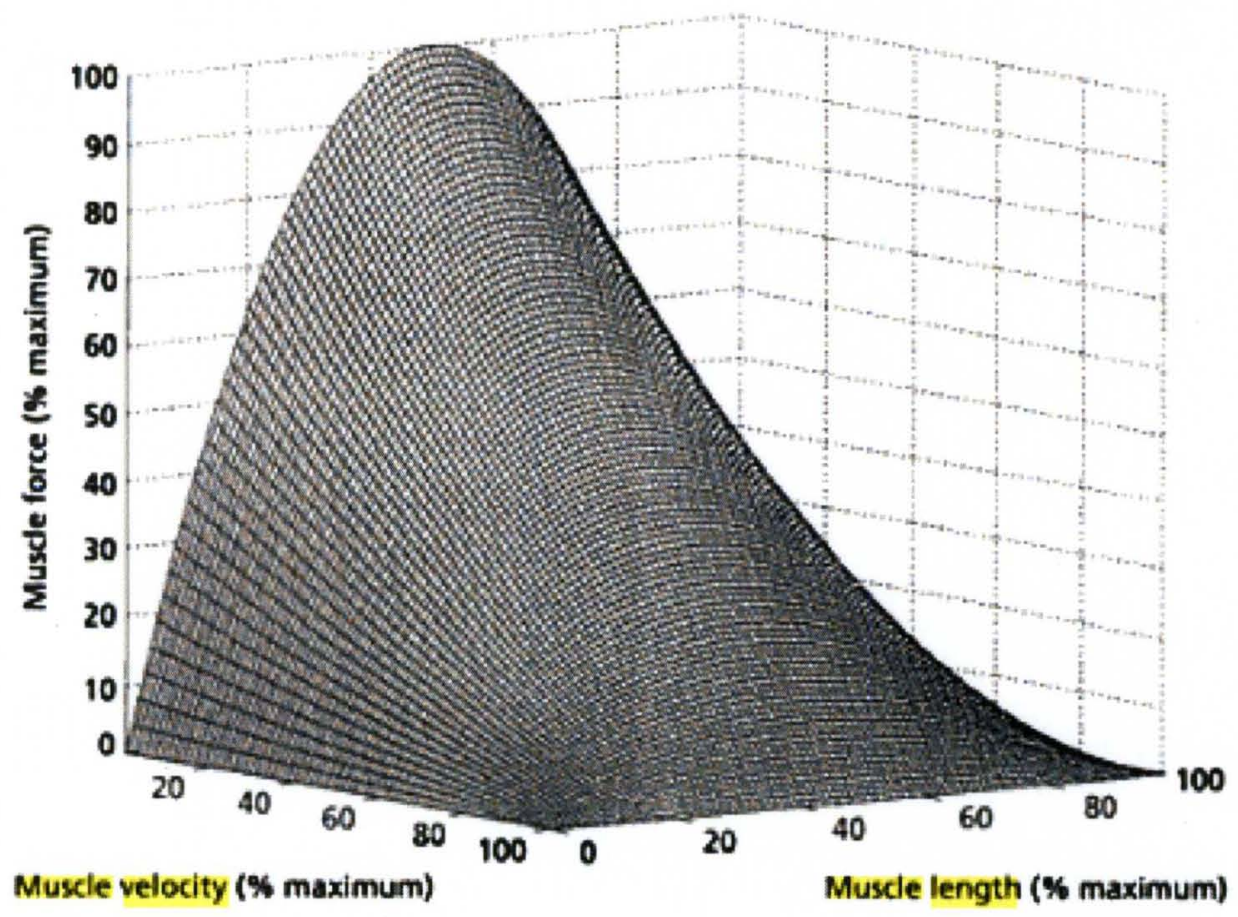

Figure 49. Force-length-velocity curve of an idealized muscle showing only the concentric phase of contraction.

Source. Zatriosky et. al. ${ }^{81}$ 


\begin{abstract}
APPENDIX - C
Hill-Huxley model of Muscle Tendon contraction in OpenSim 2.20

A.V. Hill's paper presents a classical model of muscle contraction dynamics ${ }^{37}$. It consists of a contractile element (CE) coupled with a parallel elastic element (PEE) that represents the active and passive forces of a muscle and a series elastic element (SEE) representing tendon tension as shown in Fgure 50. Generally, once muscle contraction velocities are found from experimental data, active and passive forces for individual muscles as well as muscle and tendon lengths is found.
\end{abstract}
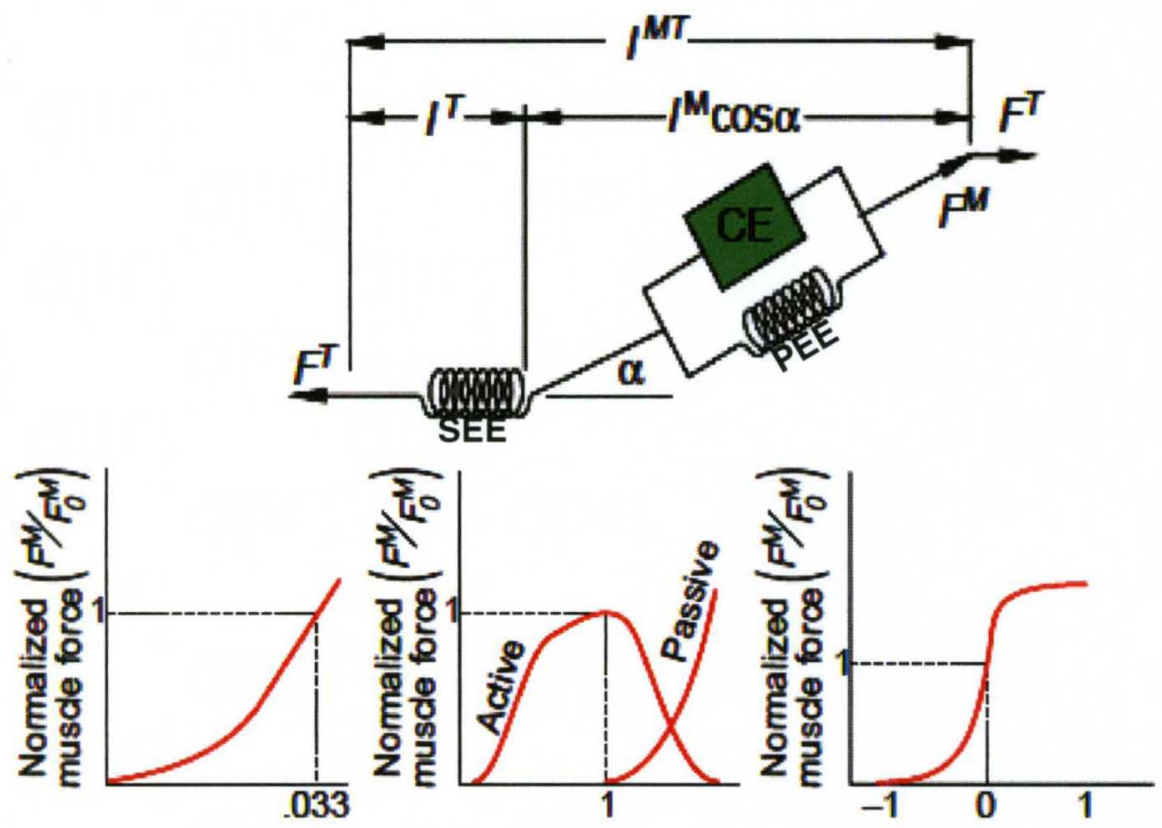

$\underset{\text { strain }}{\text { Tendon }}\left(\frac{I^{T}-I_{S}^{T}}{I_{S}^{T}}\right)$
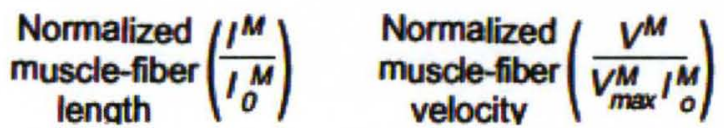

Figure 50. Hill-Huxley model of musclo-tendon contraction dynamics, as modified and used in OpenSim $2.20{ }^{64}$. 


\section{APPENDIX - D}

\section{Definition of joint kinetic labels for Figure 6}

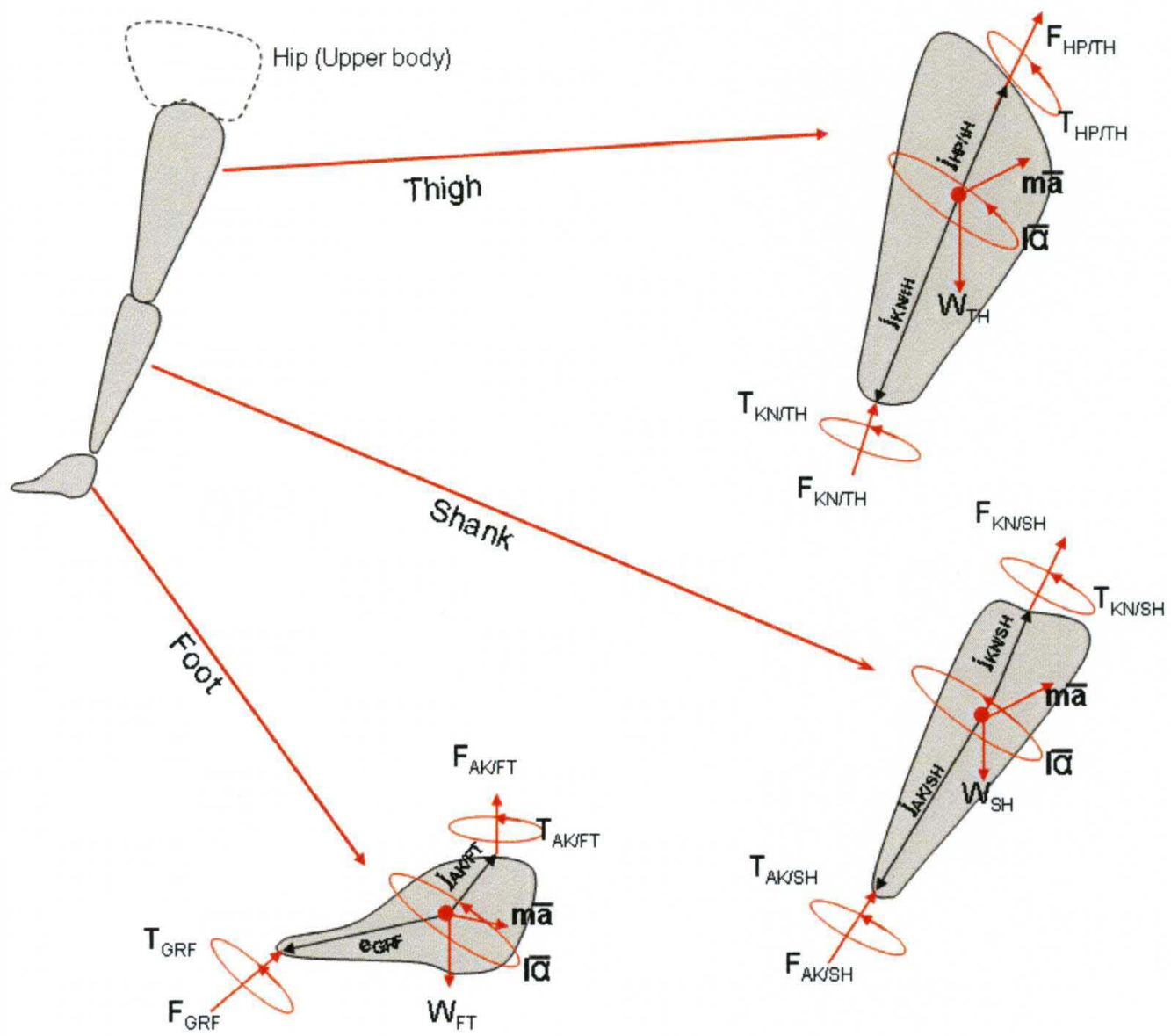

Figure 6 Repeated. Lower extremity segment and joint dynamics. Explanation of labels are given below. 


\section{Explanation of terms used in Figure 6 and equations 3-8}

\begin{tabular}{|c|c|}
\hline Label & Definition \\
\hline $\mathrm{T}_{\mathrm{GRF}}$ & Ground reaction torque. \\
\hline $\mathrm{F}_{\mathrm{GRF}}$ & Ground reaction force. \\
\hline $\mathrm{T}_{\mathrm{AK} / \mathrm{FT}}$ & External torque on foot segment applied at ankle. \\
\hline $\mathrm{F}_{\mathrm{AK} / \mathrm{FT}}$ & External force on foot segment applied at ankle. \\
\hline $\mathrm{T}_{\mathrm{AK} / \mathrm{SH}}$ & External torque on shank segment applied at ankle. \\
\hline $\mathrm{F}_{\mathrm{AK} / \mathrm{SH}}$ & External force on shank applied at ankle. \\
\hline $\mathrm{T}_{\mathrm{KN} / \mathrm{SH}}$ & External torque on shank applied at knee. \\
\hline $\mathrm{F}_{\mathrm{KN} / \mathrm{SH}}$ & External force on shank applied at knee. \\
\hline $\mathrm{T}_{\mathrm{KN} / \mathrm{TH}}$ & External torque on thigh segment applied at knee. \\
\hline $\mathrm{F}_{\mathrm{KN} / \mathrm{TH}}$ & External force on thigh applied at knee. \\
\hline $\mathrm{T}_{\mathrm{HP} / \mathrm{TH}}$ & External torque on thigh applied at hip joint. \\
\hline $\mathrm{F}_{\mathrm{HP} / \mathrm{TH}}$ & External force on thigh applied at hip joint. \\
\hline$\dot{\mathrm{H}}_{\mathrm{FT}}$ & $\begin{array}{l}\text { Time rate of change in angular momentum about foot } \\
\text { COM. }\end{array}$ \\
\hline$\dot{\mathrm{H}}_{\mathrm{SH}}$ & $\begin{array}{l}\text { Time rate of change in angular momentum about } \\
\text { shank COM. }\end{array}$ \\
\hline $\mathrm{W}_{\mathrm{FT}}, \mathrm{W}_{\mathrm{SH}}, \mathrm{W}_{\mathrm{TH}}$ & Weights of individual segments. \\
\hline & Distance of joint A from segment B COM. \\
\hline
\end{tabular}


APPENDIX - E

Given in Figures 51-56 are GRF Forces and free moments averaged from eleven subjects
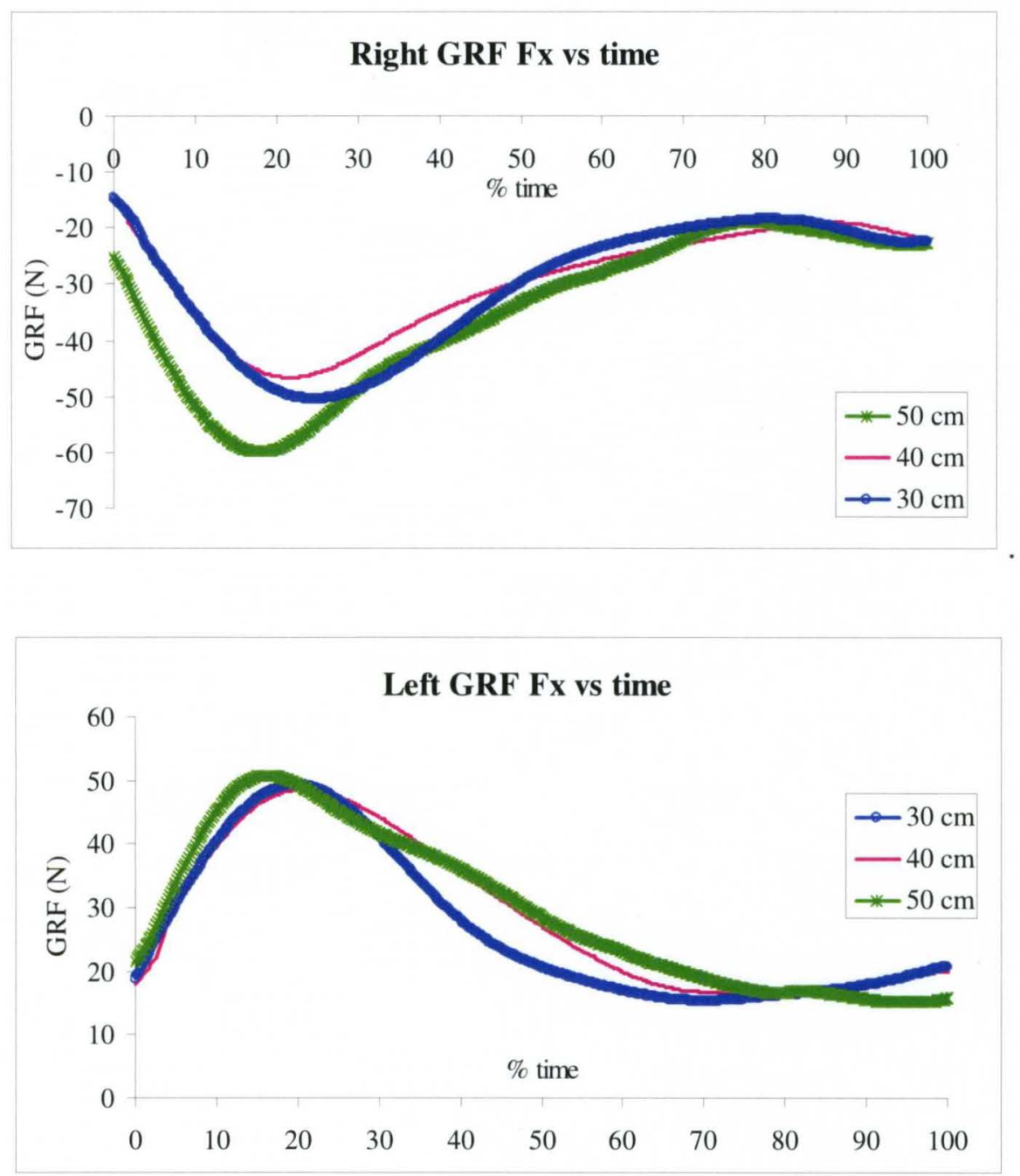

Figure 51. Average right and left GRF forces vs time in the $\mathrm{x}$ direction. 

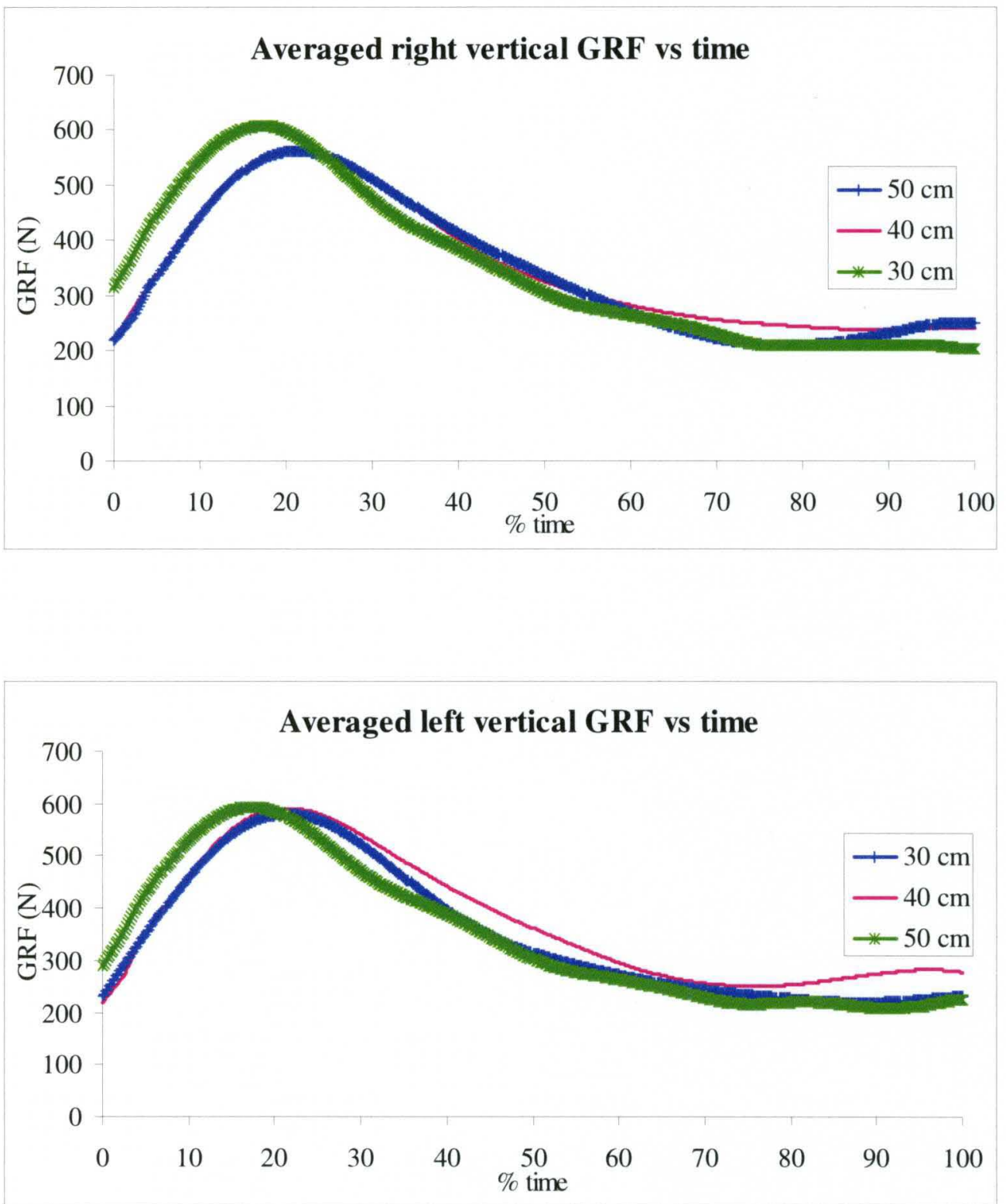

Figure 52. Average right and left GRF forces vs time in the y direction. 

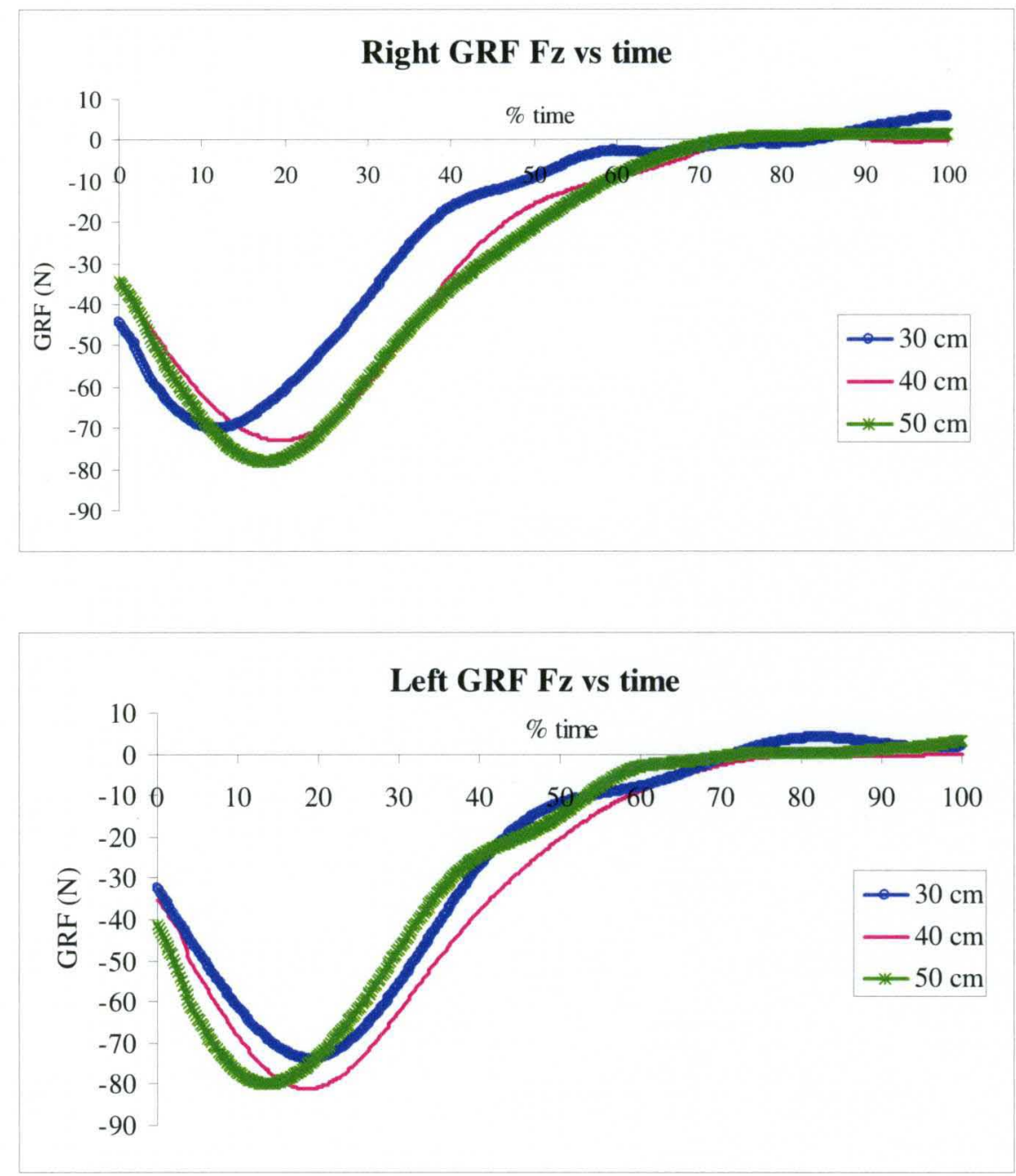

Figure 53. Average right and left GRF forces vs time in the $\mathrm{z}$ direction. 

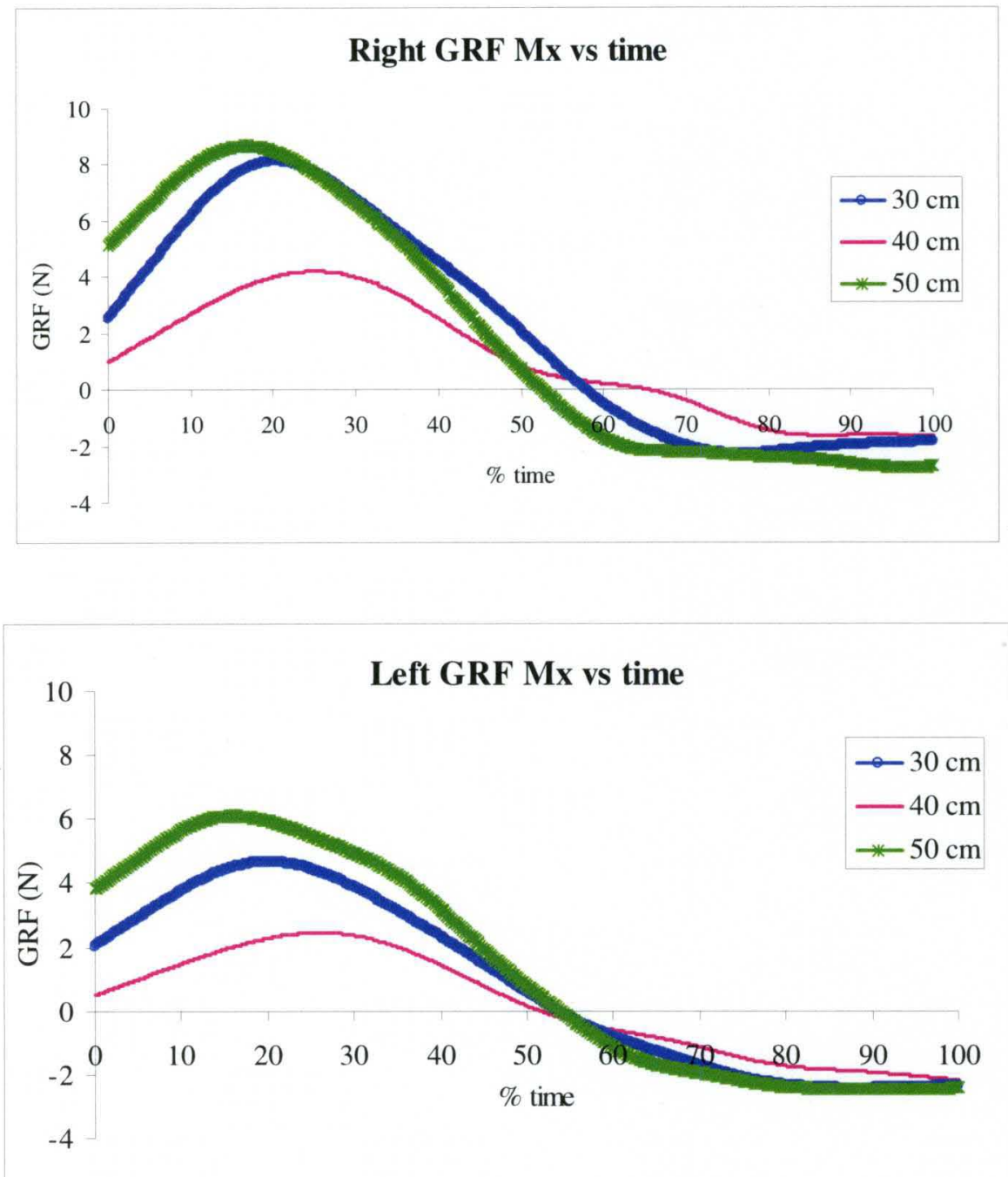

Figure 54. Average right and left GRF moments vs time in the $x$ direction. 

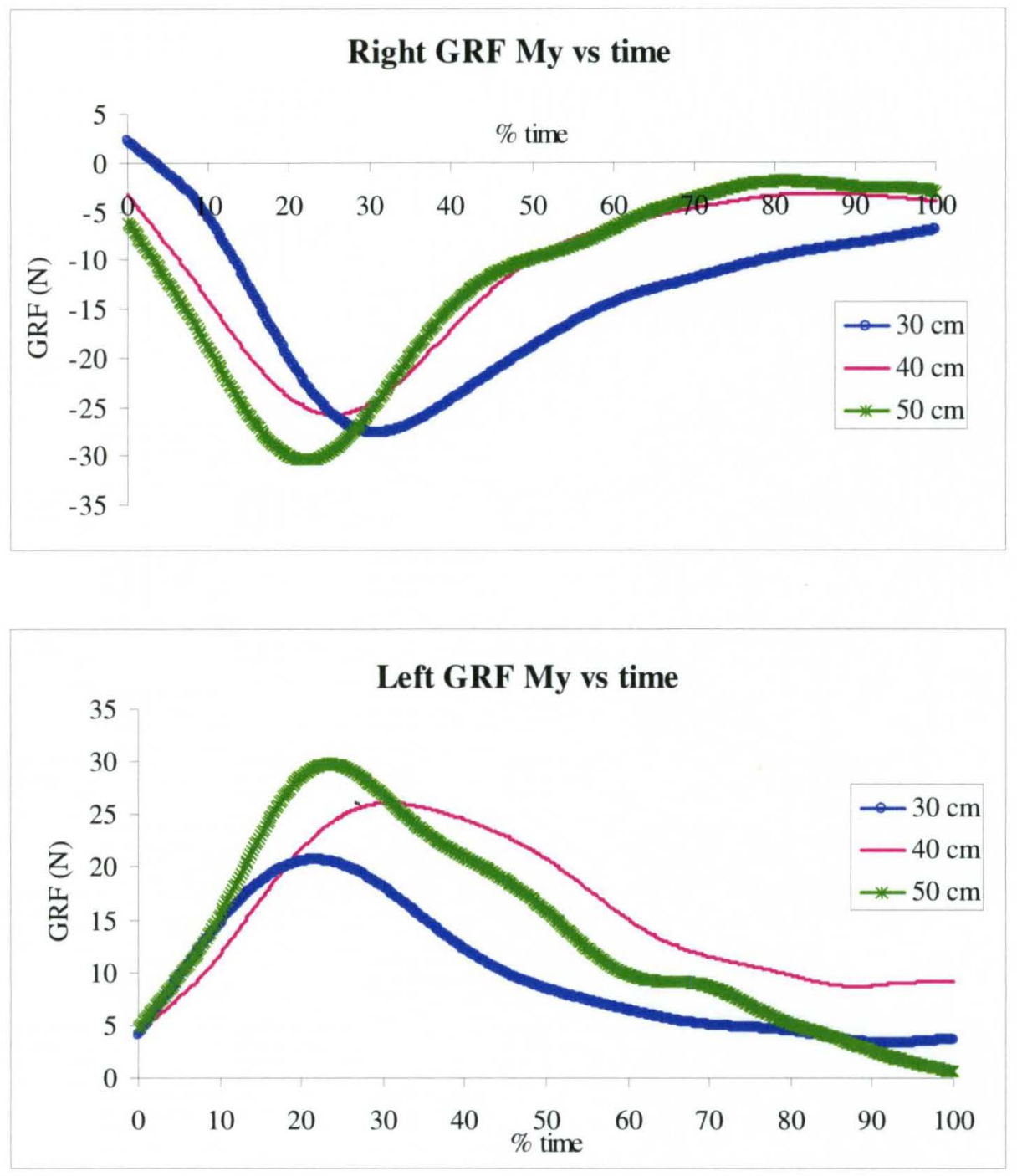

Figure 55. Average right and left GRF moments vs time in the y direction. 

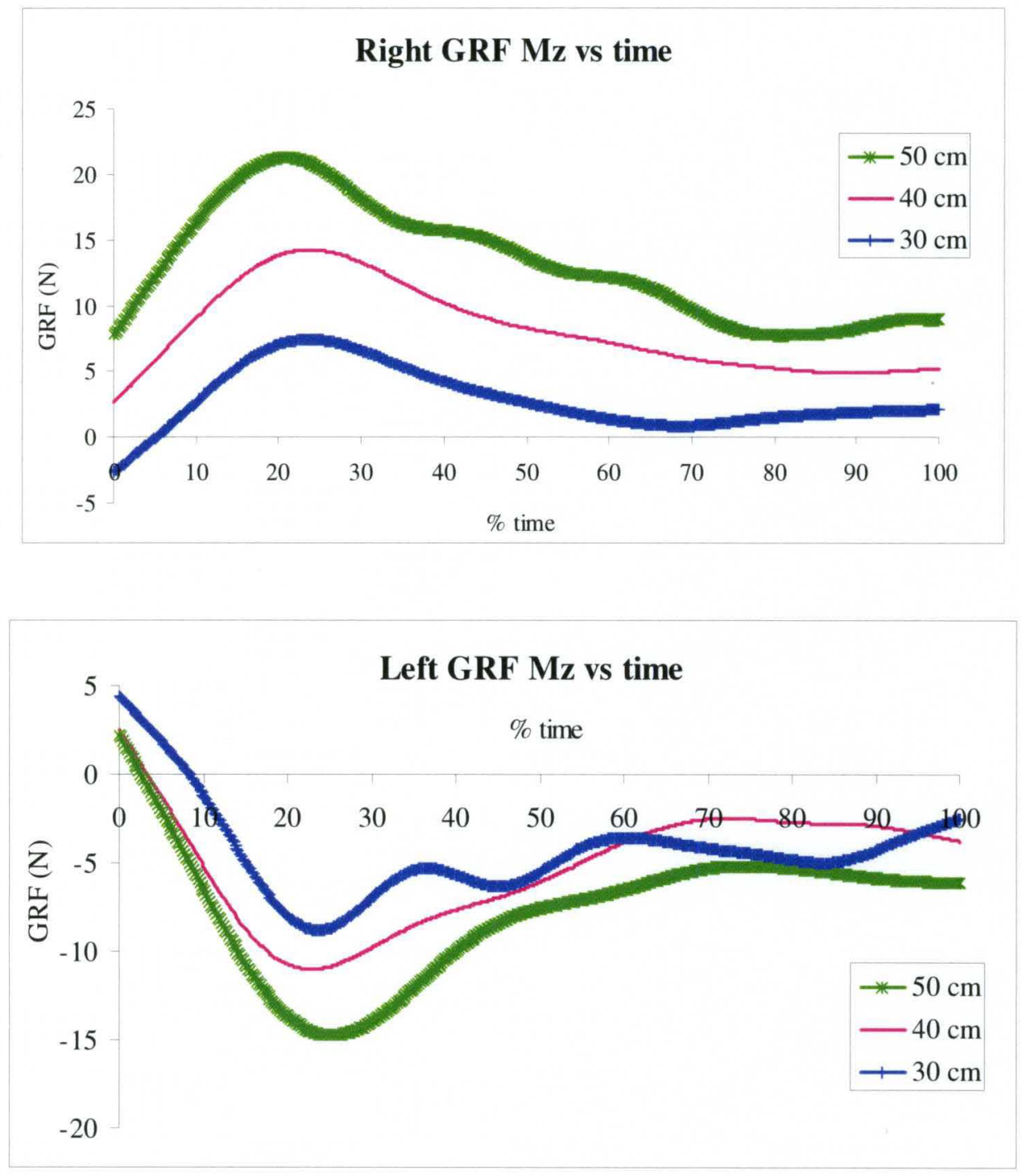

Figure 56. Average right and left GRF moments vs time in the $\mathrm{z}$ direction. 
APPENDIX - F

Ground reaction force data from drop-jump study by Burkhardt et al., 1990.

Bar plot showing small changes in ground reaction forces between 42 and $63 \mathrm{~cm}$ drop jumps performed by athletes recruited from CSU, Long Beach ${ }^{8}$.

\section{PEAK PROPULSION GROUND REACTION FORCES Relative to body weight}

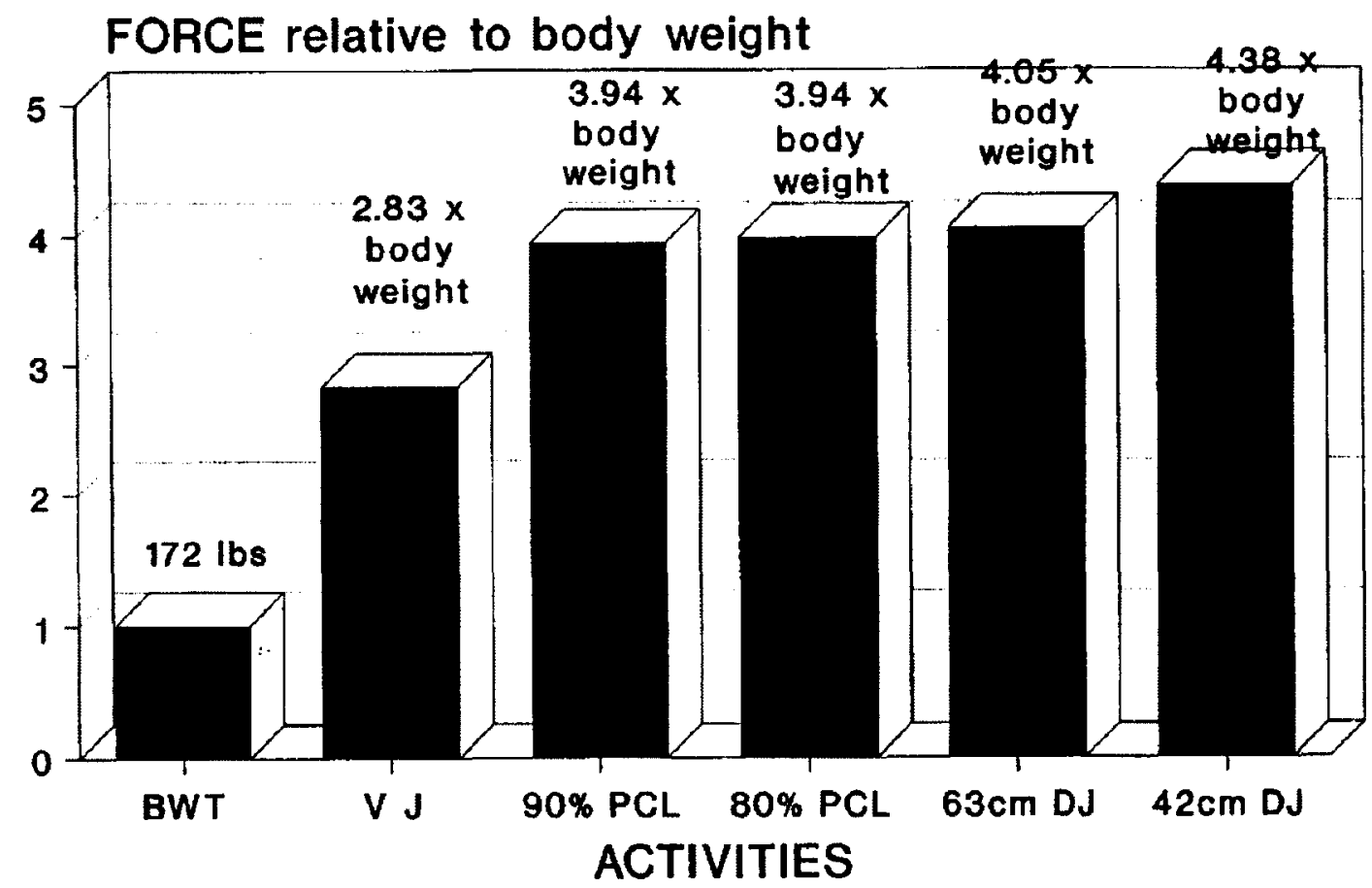

Figure 57. Peak propulsion GRF relative to body weight.

Source: Burkhardt et al. ${ }^{8}$

$\underline{\text { Terminology }}$

BWT - body weight $\quad$ VJ - vertical jump $\quad$ PCL - power clean $\quad$ DJ - drop jump 


\section{APPENDIX - G}

Strain rate data from rabbit ligament presented by Solomonow et al., 2004.

It shows how increased strain rates lead to higher tensions.

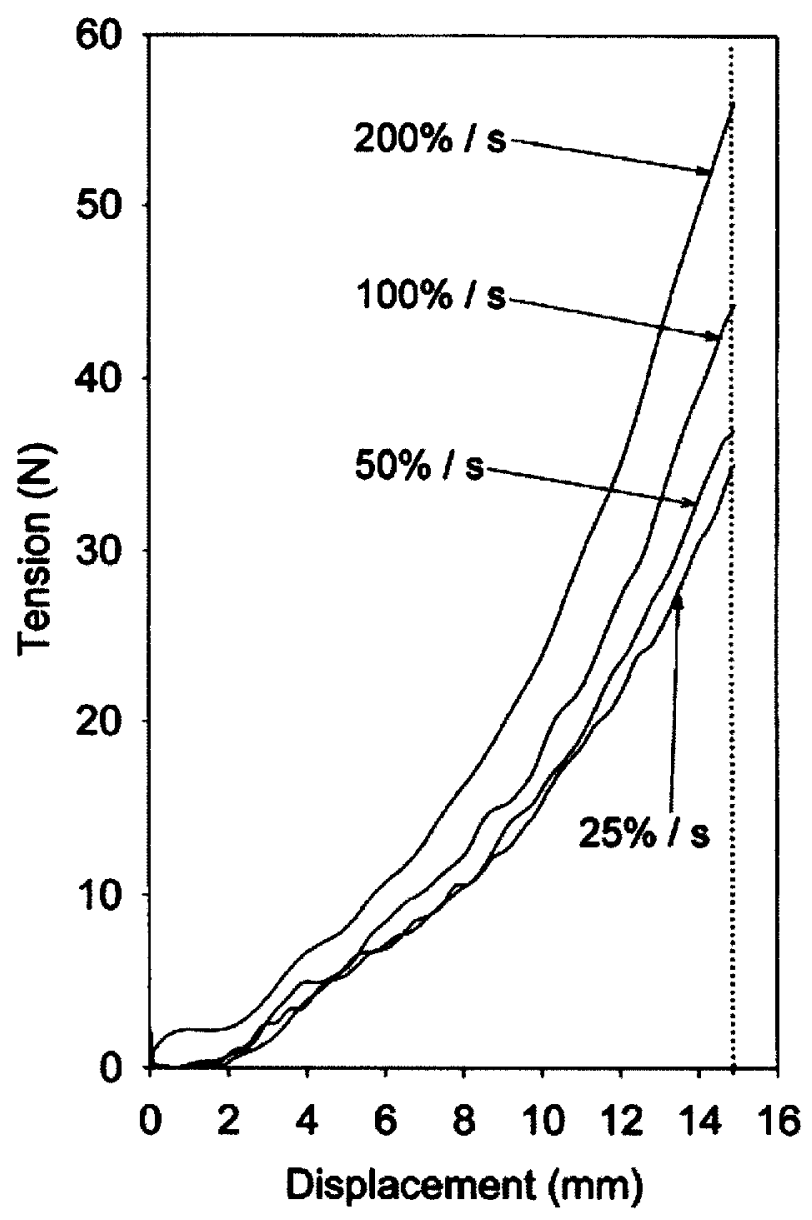

Figure 58. Length-tension relation of a (rabbit) ligament when stretched at different rates. Increasing rate of stretch increases tension.

Source: Solomonow et al. ${ }^{65}$ 


\section{CURRICULUM VITAE}

NAME: $\quad$ Julia Kar

ADDRESS: $\quad 145$ Bellerosa Dr, St Louis, MO 63122

EDUCATION Bachelors in Mechanical Engineering,

\& TRAINING: $\quad$ University of Auckland, 1996

M.S. in Mechanical Engineering (M.S.),

University of Louisville, 2008

AWARDS: Dissertation Completion Fellowship Award,

University of Louisville. 2011.

Member, Golden Key International Honor Society, 2010

Final Year Bachelor of Engg. Award, Heat Exchanger Design,

University of Auckland, 1996.

PROFESSIONAL Member, Institute of Professional Engineers,

SOCIETIES: $\quad$ New Zealand, 1997

\section{PUBLICATIONS:}

1. Moustakidis P., Pyo R., Scheri R.P., Kar J., Cupps B.P., Moulton M.J., Reddi S., Guccione J.M., Pasque M.K. Contribution of the altered geometry in the global and regional mechanical dysfunction of an ovine model of left ventricular aneurysm. Surgical Forum. 1999; 50: 169-171.

2. Dokos S., LeGrice I.J., Smaill B.H., Kar J., and Young A.A. A triaxial-measurement shear-test device for soft biological tissues. J Biomech Eng. 2000; 122: 471-478. 
3. Clark R.D., Kar J., Akella L., Soltanshahi F. OptDesign: Extending optimizable kdissimilarity selection to combinatorial library design. Journal of Chemical Information and Computer Sciences. 2003; 43: 829-836.

4. Burnett D.R., Campbell-Kyureghyan N.H., Kar J., Quesada P.M. Evaluation of asymmetry in ground reaction forces and muscle activity during the stance phase of gait in asymptomatic subjects. Proceedings of the 27th Annual Meeting of the American Society of Biomechanics, 2009. 